\title{
Abstracts of the 16th German Allergy Congress Dresden, September 30-October 2, 2021
}

Online publiziert: 3 September 2021

(C) Springer Medizin Verlag GmbH, ein Teil von Springer Nature 2021

\section{EPIDEMIOLOGY}

\section{P1.1}

\section{Alters- und geschlechtsabhängige}

Polysensibilisierungsraten gegen häufige

Inhalationsallergene in Mitteldeutschland über die letzten 20 Jahre

\section{Beutner', S. Forkel', S.Gupta', T. Fuchs', M.P. Schön ${ }^{1,2}$, J. Geier ${ }^{2,3}$, T. Buhl', 2}

${ }^{1}$ Klinik für Dermatologie, Venerologie und Allergologie, Universitätsmedizin Göttingen, Göttingen, Deutschland; ${ }^{2}$ Niedersächsisches Institut für Berufsdermatologie, Universitätsmedizin Göttingen, Göttingen, Deutschland; ${ }^{3}$ Informationsverbund Dermatologischer Kliniken (IVDK) Göttingen, Göttingen, Deutschland

Einleitung: Die Prävalenz von Atemwegsallergien in Europa ist in den letzten Jahren von $23 \%$ auf $31 \%$ gestiegen. Die Polysensibilisierung ist mit der Entwicklung und Schwergrad relevanter Allergien, insbesondere dem allergischen Asthma, assoziiert.

Ziel: Untersuchung von alters- und geschlechtsabhängigen Mono- und Polysensibilisierungsprofilen sowie Sensibilisierungsmustern mittels Hautpricktests (HPT) für die in Deutschland häufigsten Aeroallergene.

Patienten und Methoden: Retrospektiv analysiert wurden HPTs von 2886 symptomatischen Patienten mit Verdacht auf Atemwegsallergie, vorstellig 1998 bis 2017 am Universitätsklinikum Göttingen. Ausgewertet wurden in dieser Studie die wichtigen Haupt-Aeroallergengruppen frühblühende Baumpollen, Gräserpollen und Hausstaubmilben.

Ergebnisse: Zwei Drittel unserer Patienten zeigten eine Sensibilisierung gegen mindestens eine Hauptallergengruppe, davon waren $2 / 3$ polysensibilisiert. Über die letzten 20 Jahre lässt sich eine signifikante Zunahme der Polysensibilisierung in den häufigsten Aeroallergengruppen verzeichnen. Jungen und junge Männer zeigten in unserer Studiengruppe die stärkste Zunahme der Gesamtsensibilisierungsraten über die Zeit. Das häufigste Sensibilisierungsmuster stellte die Dreifachsensibilisierung gegen alle drei Hauptallergengruppen dar, mit signifikantem Anstieg von $33 \%$ auf $41 \%$ über die Zeit.

Diskussion: Diese patientenbasierte Studie zeichnet einen kontinuierlichen Anstieg der Polysensibilisierungsraten in den letzten 20 Jahren auf, wobei Jungen und junge Männer am häufigsten betroffen sind. Polysensibilisierung manifestiert sich häufig im Sinne eines multimorbiden Rhinitis-Asthma-Phänotyp. Obwohl die Daten nicht auf einer bevölkerungsbasierten Studie beruhen, deuten sie auf eine Entwicklung mit steigenden Raten von allergischen Atemwegserkrankungen hin. 


\section{P1.2}

\section{Management of patients with seasonal allergic rhinitis: Diagnostic consideration of sensitization to non-frequent pollen allergens}

\section{Höflich ${ }^{1}$, G. Balakirskiं ${ }^{2,3}$, Z. Hajdu ${ }^{4}$, J. M. Baron², K. Fietkau², H. F. Merk ${ }^{2}$, U. Strassen ${ }^{4}$, H. Bier ${ }^{4}$, W. Dott' ${ }^{5}$, H.-G. Mücke', W. Straff', G. Wurpts ${ }^{2}$, A.S. Yazdi' ${ }^{2}$, A. Chaker ${ }^{4,6}$, S. T. M. Röseler ${ }^{2,7}$}

${ }^{1}$ German Environment Agency, Section II 1.5 Environmental Medicine and Health Effects Assessment, Berlin, Germany; ${ }^{2}$ Department of Dermatology and Allergology, University Hospital RWTH Aachen, Aachen, Germany; ${ }^{3}$ Department of Dermatology, Allergology and Dermatosurgery, HELIOS University Hospital Wuppertal, University of Witten/Herdecke, Wuppertal, Germany; ${ }^{4}$ Department of Otorhinolaryngology, Klinikum rechts der Isar, Technical University Munich, Munich, Germany; ${ }^{5}$ Department for Environmental Medicine, University Hospital RWTH Aachen, Aachen, Germany;

${ }^{6}$ Center of Allergy and Environment (ZAUM), Technical University and Helmholtz Center Munich, Munich, Germany; ${ }^{7}$ Department of Pneumology, Allergology, Sleep and Respiratory Medicine, Augustinians Hospital, Cologne, Germany

In the minimum skin prick test battery recommended by the Global allergy and asthma European Network ten pollen allergens are included. Complementary, other pollen allergens may need to be considered, however, respective awareness may not always be granted. Furthermore, at least in Germany, the situation may be even more complicated by the fact that test allergens need regulatory approval. A decline of commercially available test allergens may result in a diagnostic gap regarding patients with non-frequent allergies.

Using skin prick testing and/or ISAC technology, sensitization to frequent pollen allergens (alder, hazel, birch, sweet grasses; according to the German Therapy Allergen Ordinance) and to respective non-frequent pollen allergens (cypress, Japanese cedar, ash, plane tree, olive, Bermuda grass, wall pellitory, plantain, goosefoot, mugwort, ragweed and saltwort) were measured in adult patients with physician-diagnosed allergic rhinitis from two German federal states, namely North-Rhine Westphalia $(n=360)$ and Bavaria $(n=339)$. Sensitization data were completed by anamnestic data on symptom periods and by respective regional pollen data.

Sensitization to frequent pollen allergens ranged from $45 \%$ (sIgE to Aln g 1/alder, NRW) to $72 \%$ (prick test reactivity to birch, NRW). Sensitization to non-frequent pollen allergens ranged from $0 \%$ (sIgE to Amb a 1/ragweed, NRW) to $41 \%$ (prick test reactivity to olive, Bavaria). Sensitization data partly correlated with each other and in connection with symptom periods showed a partly similar seasonal pattern as pollen data.

Sensitization to non-frequent pollen allergens have to be considered when examining patients with respective seasonal symptoms, and test (and therapy) allergens for non-frequent pollen allergies need to be available. Further prerequisites for adequate patient management would be a nationwide pollen monitoring system giving continuous pollen data and a systematic sensitization monitoring at patient level.

\section{P2.1}

Type 2 inflammation related comorbidities among patients with asthma, chronic rhinosinusitis with nasal polyps, and atopic dermatitis

\section{A. H. Khan', I. Gouia', S. Kamat', M. Bastian', B. Ortiz²,} R. Johnson ${ }^{4}$, J. Siddall ${ }^{5}$, M. Small ${ }^{5}$

'Sanofi, Chilly Mazarin, France;

${ }^{2}$ Regeneron Pharmaceuticals, Inc., Tarrytown, NY, USA;

${ }^{3}$ Sanofi, Frankfurt, Germany;

${ }^{4}$ Sanofi, Bridgewater, NJ, USA;

${ }^{5}$ Adelphi Real World, Bollington, UK

Background: Type 2 inflammation is implied in the pathogenesis of several coexisting diseases. The aim of this study was to estimate the prevalence of common co-existing Type 2 inflammatory diseases in patients (pts) with moderate-tosevere (m/s) Asthma, Chronic Rhinosinusitis with Nasal Polyps (CRSwNP) or Atopic Dermatitis (AD). Additionally, the severity of Type 2 comorbidities in m/s Asthma and m/s CRSwNP pts was estimated.

Methods: A convenience sample of physicians from the US and EU5 (France, Germany, Italy, Spain and the UK) were contacted (JAN2017-FEB2020) to identify and review medical records of pts with $\mathrm{m} / \mathrm{s}$ Asthma, $\mathrm{m} / \mathrm{s}$ CRSwNP, or m/s AD. Based on physician judgement, comorbidities linked to Type 2 inflammation and severity of comorbidities were reported.

Results: 761 physicians identified pts with $\mathrm{m} / \mathrm{s}$ Asthma ( $n=899), \mathrm{m} / \mathrm{s}$ CRSwNP $(n=683)$, or $\mathrm{m} / \mathrm{s} \mathrm{AD}(n=367)$. Among $\mathrm{m} / \mathrm{s}$ Asthma pts, respectively $60 \%, 15 \%, 17 \%$ had allergic rhinitis (AR), AD, CRSwNP. Among m/s CRSwNP pts, respectively $46 \%, 53 \%, 9 \%$ had asthma, AR, AD. Among m/s AD patients, respectively $31 \%, 41 \%$, and $3 \%$ had asthma, AR, CRSwNP. In $\mathrm{m} / \mathrm{s}$ Asthma and $\mathrm{m} / \mathrm{s}$ CRSwNP pts, a high proportion of Type 2 comorbidities presented as $\mathrm{m} / \mathrm{s}$ disease.

Conclusions: High proportions of pts with $\mathrm{m} / \mathrm{s}$ Asthma, $\mathrm{m}$ / $\mathrm{s} A D$, and $\mathrm{m} / \mathrm{s}$ CRSwNP had other co-existing Type 2 inflammatory diseases. Given the high comorbidity burden, an integrated treatment approach addressing underlying Type 2 inflammation should be considered. 
Changes in mast cell (MC) numbers and phenotype in patients (pts) with indolent systemic mastocytosis (ISM) treated with avapritinib

K. Hartmann 1 , T. George ${ }^{2}$, S. Broesby-Olsen ${ }^{3}$, D. Wada ${ }^{4}$, S. Florell ${ }^{4}$, F. Siebenhaar ${ }^{5}$, C. Akin ${ }^{6}$, K. Newberry ${ }^{7}$, H. Shi ${ }^{7}$, M. Roche ${ }^{7}$, M. Maurer ${ }^{8}$, H. O. Elberink ${ }^{9}$

${ }^{1}$ University of Basel, Basel, Switzerland;

${ }^{2}$ ARUP Laboratories, University of Utah, Salt Lake City, Utah, USA;

${ }^{3}$ Odense University Hospital, Odense, Denmark;

${ }^{4}$ University of Utah, Salt Lake City, Utah, USA;

${ }^{5}$ Charité - Universitätsmedizin Berlin, corporate member of

Freie Universität Berlin, Humboldt-Universität zu Berlin, and Berlin Institute of Health, Berlin, Germany;

${ }^{6}$ University of Michigan, Ann Arbor, Michigan, USA;

${ }^{7}$ Blueprint Medicines Corporation, Cambridge,

Massachusetts, USA;

${ }^{8}$ Charité - Universitätsmedizin Berlin, corporate member of

Freie Universität Berlin, Humboldt-Universität zu Berlin, and

Berlin Institute of Health, Berlin, German;

'University Medical Center Groningen, University of

Groningen, Groningen; Netherlands

Purpose: Avapritinib, a potent and selective KIT D816V inhibitor, has improved skin lesions in pts with ISM in Part 1 of the placebo-controlled PIONEER (NCT03731260) study. Here we assessed the impact of avapritinib on number and immunophenotype of MCs in bone marrow (BM) and skin biopsies from 39 pts participating in PIONEER Part 1.

Methods: BM biopsies and aspirates were obtained at Screening; Lesional (LT) and non-lesional (NLT) skin biopsies were obtained at Screening and end of Week 12 (W12). Immunohistochemistry was performed on formalin-fixed sections with antibodies against CD117, tryptase, CD25, CD30, and CD34. Samples were centrally reviewed by three pathologists. Statistical significance of MC numbers and immunophenotypic changes were assessed. Data cut-off was December 4, 2020.

Results: Screening BM biopsies had mean (SD) $15.7 \%$ (15.5\%) proportion of MCs, of which $74.2 \%(23.6 \%)$ were spindled. BM aspirates had mean (SD) $2.8 \%$ (3.1\%) MCs, of which $4.3 \%(1.7 \%)$ were immature. Avapritinib reduced the mean (SD) MCs $/ \mathrm{mm} 2$ in the skin in LT from 702.8 (961.0) at Screening to 233.9 (216.5) at W12; mean number in NLT remained stable from 115.2 (98.3) at Screening to 103.5 (42.3) at W12. BM biopsies had higher mean (SD) proportions of CD25 + $(77.6 \%$ [7.2\%]) and CD30 + MCs (52.8\% [31.9\%]) compared with skin LT (mean [SD] CD25 +, $5.9 \%$ [10.8\%]; CD30 +, $26.9 \%$ [29.8\%]) and NLT (CD25 +, $1.8 \%$ [1.5\%]; CD30 +, $4.3 \%$ [6.9\%]). Avapritinib significantly reduced CD30 + MCs in LT at W12 (mean [SD] $1.6 \%$ [1.4\%]) vs placebo $(13.3 \%[13.0 \%])(P .=0.0082)$ and numerically reduced CD30 + MCs in NLT at W12 $(1.2 \%[0.4 \%])$ vs placebo $(2.4 \%[3.4 \%])(P=0.0821)$.

Conclusions: Avapritinib decreased total MC numbers in skin LT compared to placebo. In particular, avapritinib reduced CD30 + MCs in skin LT. Hence, in this first study examining changes in MCs numbers and immunophenotype during precision therapy of ISM, we demonstrated efficacy of avapritinib in reducing clonal MCs in the skin in ISM.

\section{Betalaktam-Antibiotika assoziierte \\ Überempfindlichkeitsreaktionen: deskriptive Analyse von Berichten zu unerwünschten Arzneimittelreaktionen aus Deutschland}

\section{Dubralli, 2, M. Schulz ${ }^{3}$, M. Schmid ${ }^{1}$, B. Sachs ${ }^{2,4}$}

${ }^{1}$ Institut für Medizinische Biometrie, Informatik und Epidemiologie, Uniklinikum Bonn, Bonn, Deutschland; ${ }^{2}$ Bundesinstitut für Arzneimittel und Medizinprodukte, Bonn, Deutschland;

${ }^{3}$ Zentralinstitut für die kassenärztliche Versorgung in der Bundesrepublik Deutschland, Berlin, Deutschland; ${ }^{4}$ Klinik für Dermatologie und Allergologie, Uniklinik RWTH Aachen, Aachen, Deutschland

Einleitung: Überempfindlichkeitsreaktionen sind bekannte unerwünschte Arzneimittelreaktionen (UAW) von Betalaktam-Antibiotika (BLA). Verglichen mit Penicillinen, wurden Cephalosporin-assoziierte Überempfindlichkeitsreaktionen selten untersucht. Zudem ist die Vergleichbarkeit der Studien durch Unterschiede im Studiendesign erschwert. Oft wurden auch die Verordnungen nicht berücksichtigt. Das Ziel unserer Studie war, Berichte zu Überempfindlichkeitsreaktionen stratifiziert nach BLA zu untersuchen und deren Anzahl im Verhältnis zur Anzahl an Verordnungen zu betrachten.

Methoden: In der europäischen UAW-Datenbank EudraVigilance wurden 1387 Berichte aus Deutschland zu BLA-assoziierten Überempfindlichkeitsreaktionen im Zeitraum 2010-2018 identifiziert. Die Berichte wurden stratifiziert nach BLA analysiert und ins Verhältnis zur Anzahl an Verordnungen gesetzt.

Ergebnisse: Cephalosporin-assoziierte Überempfindlichkeitsreaktionen waren im Vergleich zu den Penicillinen öfter als schwerwiegend klassifiziert. Zudem traten anaphylaktische Reaktionen bei den Cephalosporinen der ersten (52.6\%), zweiten (33.2\%) und dritten (9.1\%) Generation wesentlich häufiger auf als bei den Penicillinen (4.7\%). Auch die bullösen Hautreaktionen waren ungefähr drei- bzw. zweimal häufiger für Cephalosporinen der dritten und ersten Generation berichtet verglichen mit den Penicillinen und Cephalosporinen der zweiten Generation. Im Verhältnis zur Anzahl an Verordnungen lagen mehr Berichte zu den Cephalosporinen der ersten Generation vor, gefolgt von den Penicillinen und den Cephalosporinen der dritten und zweiten Generation.

Schlussfolgerung: Unterschiede in Bezug auf den Schweregrad sowie der Art der Überempfindlichkeitsreaktion wurden in der BLA-stratifizierten Analyse beobachtet. Als Ursache kommen auch Unterschiede im Berichtsverhalten oder in den Patientenpopulationen in Frage. Um unsere Ergebnisse zu bestätigen sind weitere Untersuchungen notwendig. 


\section{ALLERGIC RHINITIS}

\section{P1.3}

\section{Sensibilisierung gegenüber Götterbaum (Ailanthus altissima) in einer Hochrisikopopulation}

\section{F.Prenzel ${ }^{1,2}$, M. van Hove ${ }^{1,2}$, P. Kage ${ }^{2,3}$, T. Lipek ${ }^{1,2}$, M. Bastl', T. Kaiser ${ }^{2,5}$, S. Kuhs ${ }^{2,5}$, M. Boege ${ }^{2,6}$, J. Zarnowski ${ }^{2,3}$, J. Genuneit', J.-C. Simon ${ }^{2,3}$, S. Dunker ${ }^{7,8}$, R. Treudler ${ }^{2,3}$}

${ }^{1}$ Universitätsklinik für Kinder- und Jugendmedizin Leipzig, Leipzig, Deutschland;

${ }^{2}$ Leipziger interdisziplinäres Centrum für Allergologie (LICA), Leipzig, Deutschland;

${ }^{3}$ Universitätsklinik für Dermatologie, Venerologie und Allergologie, Leipzig, Deutschland;

${ }^{4}$ Universitätsklinik für Hals-, Nasen- und Ohrenkrankheiten, Wien, Österreich;

${ }^{5}$ Institut für Laboratoriumsmedizin, Klinische Chemie und Molekulare Diagnostik, Universitätsklinik Leipzig, Deutschland;

${ }^{6}$ Universitätsklinik für Hals-Nasen-Ohrenheilkunde, Leipzig, Deutschland;

${ }^{7}$ Helmholtz-Zentrum für Umweltforschung (UFZ), Department Physiologische Diversität, Leipzig, Deutschland;

${ }^{8}$ Deutsches Zentrum für integrative Biodiversitätsforschung, Leipzig, Deutschland

Hintergrund: Götterbaum, ein in China heimischer Laubbaum, wächst in Europa stark invasiv. Pollen wird lokal und aerogen verbreitet. Eine hohe Sensibilisierungsrate wurden in einer chinesischen Kohorte nachgewiesen. In Leipzig wachsen große Götterbaum-Bestände. Wir haben gefragt: 1) Können in einer Pollenfalle im Zentrum von Leipzig Götterbaumpollen nachgewiesen werden? 2) Sind bei den im CAC behandelten Patienten Sensibilisierungen gegen Götterbaum nachzuweisen?

Methoden: Eine Hirst-Burkhard-Pollenfalle wurde 4/2019 auf dem Dach des Uniklinikums in Leipzig (Höhe $17.3 \mathrm{~m}$ ) aufgestellt. Das Fass wurde wöchentlich gewechselt und die Pollen mikroskopisch ausgezählt. In asserviertem Serum von Patienten unseres CAC wurde IgE gegen Götterbaum und die kreuzreaktiven Allergene Bet v2 und MUXF3 bestimmt (Phadia 250 ImmunoCAP ${ }^{\circledR}$-System). Die Kohorte wurde 2019-2020 nach den Kriterien ausgewählt: 1) Diagnose allergische Rhinitis (AR) und/oder Asthma und/oder Atopische Dermatitis (AD) mit Symptomen Mai - Juli 2) Wohnort in Leipzig Die Daten wurden retrospektiv aus den Akten entnommen und analysiert.

Ergebnisse: In der Pollenfalle wurden Götterbaum-Pollen von Juni bis Juli mit einer maximalen Konzentration von 31 Pollen $/ \mathrm{m}^{3}$ nachgewiesen. Es wurden 138 Patienten (69 Kinder) analysiert $(25,3 \pm 19,7$ Jahre). Von diesen hatten 95 (69\%) eine AR, 84 (61 \%) ein Asthma und 43 (31 \%) eine AD. Bei $59(42 \%)$ wurde eine Sensibilisierung gegen Götterbaum nachgewiesen, davon 27 Kinder. Die Sensibilisierungen wurden in allen Altersgruppen nachgewiesen. Bei 35 (59\% der Sensibilisierten) war kein IgE gegen die kreuzreaktiven Allergene vorhanden.

Schlussfolgerungen: Bei $42 \%$ der Patienten einer Hochrisikopopulation ließ sich eine Sensibilisierung gegen Götterbaumpollen nachweisen. Dies spricht für das allergene Potential dieses Neophyten, was bei der Anpflanzung und differentialdiagnostisch berücksichtigt werden muss. Mittels Provokationsverfahren wäre zu prüfen, ob manifeste Allergien vorliegen.

\section{P1.4}

A three-sided approach to evaluate bovine beta-lactoglobulin in a lozenge - targeted micronutrition in patients with allergic rhinoconjunctivitis

K.-C. Bergmann', 2, A. Sperl' ${ }^{3}$, L. Krause ${ }^{4}$, S. Becker ${ }^{2}$, T. Zuberbier ${ }^{1,2}$, V. B. Ott ${ }^{5}$, A. Grässel ${ }^{5}$, M. F. Kramer ${ }^{5,6}$, F. Roth-Walter ${ }^{7,8,9}$, E. Jensen-Jarolim ${ }^{7,8,9}$, S. Güthoff ${ }^{5}$, J. Raab $^{5}$

${ }^{1}$ Comprehensive Allergy-Centre-Charité, CharitéUniversitätsmedizin Berlin, Berlin, Germany;

${ }^{2}$ European Centre for Allergy Research Foundation, Berlin, Germany;

${ }^{3}$ Zentrum für Rhinologie und Allergologie, Wiesbaden,

Germany:

${ }^{4}$ Institute of Medical Biometry and Epidemiology, University

Medical Centre Hamburg-Eppendorf, Hamburg, Germany;

${ }^{5}$ Bencard Allergie GmbH, Munich, Germany;

${ }^{6}$ Allergy Therapeutics, Worthing, UK;

${ }^{7}$ Biomedical International R+D GmbH, Vienna, Austria;

${ }^{8}$ The Interuniversity Messerli Research Institute of the University of Veterinary Medicine Vienna, Medical University Vienna and University Vienna, Vienna, Austria;

Institute of Pathophysiology and Allergy Research, Centre of Pathophysiology, Infectiology and Immunology, Medical University of Vienna, Vienna, Austria

Background: Atopy is associated with nutritional deficiencies. The bovine protein beta-lactoglobulin (BLG) combined with micronutrients (holo-BLG) targets and nourishes immune cells. A holo-BLG lozenge already demonstrated positive clinical effects in allergic patients. To further investigate the allergen-nonspecific effects of holo-BLG, we used three different study approaches.

Methods: 1) 32 house dust mite (HDM)-allergic patients were challenged in an allergen exposure chamber (AEC) before and after 3 months of daily supplementation (suppl.) with the holo-BLG lozenge. Primary endpoint was the difference in Total Nasal Symptom Score (TNSS) after 120 min of HDM exposure. Symptoms of nose, eyes, bronchi and other were depicted as median Total Symptom Score (TSS); 2) 71 patients allergic to birch pollen, HDM and/or animal dander were monitored using an online survey with regards to subjective effects during 3 months of suppl.; 3) an observational study is conducted to assess the robustness of clinical effects (rhinoconjunctivitis score, combined symptom and medication score) after 3 months suppl. with holo-BLG.

Results: In the AEC study, the median TNSS was significantly reduced by $60 \%$ ( $p=0.0034)$ after suppl. with holo-BLG, compared to baseline. As an important secondary outcome measure, the TSS was improved by $40 \%(p<0.0003)$. In the online survey, $68 \%$ of participants reported that their allergic symptoms or general well-being was improved after 3 months of suppl.; $93 \%$ of participants described the tolerability of the lozenge as very good or good. The third study currently investigates 28 adults and 9 children allergic to birch pollen or HDM and/or animal dander. Allergic symptoms and medication use will be documented daily over the entire suppl. period.

Conclusion: The excellent tolerability and significant symptom improvement after only 3 months of suppl. with the lozenge contribute to the evidence of this newly developed 'food for special medical purpose'. 
P1.5

Memory effects of the holo-BLG lozenge in house dust mite allergic patients - follow-up study in an allergen exposure chamber

\section{K.-C. Bergmann ${ }^{1,2}$, J. Raab ${ }^{3}$, L. Krause ${ }^{4}$, S. Becker', S. Kugler ${ }^{2}$, T. Zuberbier ${ }^{1,2}$, F. Roth-Walter ${ }^{5,6,7}$, E. Jensen-Jarolim ${ }^{5,6,7}$, M. F. Kramer ${ }^{3,8}$, A. Graessel ${ }^{3,8}$}

${ }^{1}$ Department for Dermatology and Allergy, CharitéUniversitätsmedizin Berlin, corporate member of Freie Universität Berlin, Humboldt-Universität zu Berlin, and Berlin Institute of Health, Berlin, Germany;

${ }^{2}$ ECARF-European Centre for Allergy Research Foundation, Berlin, Germany;

${ }^{3}$ Bencard Allergie GmbH, Munich, Germany; ${ }^{4}$ Institute of Medical Biometry and Epidemiology, University Medical Center Hamburg-Eppendorf, Hamburg, Germany; ${ }^{5}$ The Interuniversity Messerli Research Institute of the University of Veterinary Medicine Vienna, Medical University Vienna and University Vienna, Vienna, Austria;

${ }^{6}$ Institute of Pathophysiology and Allergy Research, Center of Pathophysiology, Infectiology and Immunology, Medical University of Vienna, Vienna, Austria;

${ }^{7}$ Biomedical International R+D GmbH, Vienna, Austria;

${ }^{8}$ Allergy Therapeutics (UK) Ltd,Worthing, UK

Background: The holo-BLG (beta-lactoglobulin) lozenge targets micronutritional deficiencies in immune cells of allergic patients. Previous studies have shown that holo-BLG supplementation (suppl.) could improve symptoms of pollen and house dust mite (HDM) allergic patients. Our previous study documented $60 \%(p=0.0034)$ improvement of the Total Nasal Symptom Score (TNSS) after 3 months holo-BLG suppl. in HDM allergic patients after a controlled provocation in an allergen exposure chamber (AEC). To measure long-term memory effects of the holo-BLG suppl., a follow-up visit was initiated and patients from our previous study were asked to undergo another visit in the AEC.

Methods: After 7-8 months without holo-BLG suppl. (visit 5 , V5), 27 of 32 HDM allergic patients of our previous study were again exposed to HDM (Dermatophagoides pteronyssinus and farinae $-1: 1$, faeces and body allergens, $250 \mu \mathrm{g} / \mathrm{m}^{3}$ ) for $120 \mathrm{~min}$ in the AEC under the same standardized conditions $\left(21^{\circ} \mathrm{C}, 55 \%\right.$ relative air moisture). Symptoms for nose, eye, bronchi and others were recorded every $10 \mathrm{~min}$ and summed up to the total symptom score (TSS).

Results: At V5 the TNSS (primary endpoint of our previous study) was improved by $20 \%(p=0.1516)$ compared to V1 (before suppl.). The TSS, one secondary endpoint of the previous study, was improved by $40 \%(p=0.004)$ at V5, comparable to the reduction at the end of the suppl. with holo-BLG for 3 months (V3, $40 \%$ reduction, $p<0.0003$ ). The exploratory analysis of the temporal evolution of all symptom scores with mixed effects models demonstrated clinically meaningful improvement of symptoms, manifested in a lower symptom increase upon HDM challenge during V5 compared to V1.

Conclusion: Seven to 8 months after suppl. patients still experienced improvement of their allergic symptoms during the controlled exposure in the AEC with HDM compared to symptoms before holo-BLG suppl. Hence the holo-BLG lozenge provides a form of immunological memory, likely via innate mechanisms.

\section{P1.6}

Die Effektivität von „AllergoEye“, eines vollautomatischen Systems basiert auf künstlicher Intelligenz, bei der Objektivierung des konjunktivalen Provokationstestes

\section{Y. Yarin, A. Kalaitzidou, K. Bodrova, I. Kalaitzidis}

HNO Praxis Dr. Yarin, Dresden, Deutschland

Hintergrund: Die Bedeutung des konjunktivalen Provokationstests (KPT) in der Allergologie nimmt aufgrund seiner Einfachheit, Präzision und Sensitivität stetig zu. Bisher gab es jedoch keine einfache Möglichkeit, eine allergische Reaktion durch eine konjunktivale Provokation quantitativ zu analysieren und zu bestimmen.

Methode: Die auf neuronalen Netzen und künstlicher Intelligenz basierende Plattform „AllergoEye“ wurde entwickelt, um die allergische Reaktion der Sclera vollautomatisch, schnell und einfach zu quantifizieren. „AllergoEye“ besteht aus einer Android-basierten Kamera mit gesteuertem Lichtstativ und einem PC-basierten KI-Servermodul. Diese bietet dem Arzt Echtzeitsteuerung für eine vollautomatische quantitative Auswertung. Es gibt Schnittstellen, die einen Datenexport aus dem KI-Modul in Statistik- und oder Medizinsoftware ermöglichen.

Ergebnisse: Die präklinische Untersuchung an 9 Patienten, welche eine subkutane Immuntherapie im Jahre 2020 begonnen hatte, zeigte bereits nach 3 Monaten eine Effektivität durch die Senkung des Summationssymptomscores. Diese Effektivität wurde zusätzlich, während des KPT, durch eine starke Korrelation zwischen Therapieeffektivität, Reduktion der Rötung und die Quantifizierung in den Ergebnissen des „AllergoEye“ bestätigt. Die Ergebnisse zeigten, dass durch einen Arzt oder das medizinische Personal nur eine geringe signifikante Verbesserung, bei der die subjektive Rötungsbestimmung der Sklera nach 3 Monaten der Therapie, festgestellt werden konnten. „AllergoEye" dagegen lieferte Ergebnisse, welche die statistisch signifikante Senkung die Rötung der Sklera zeigen. Damit ist die Genauigkeit und Sensibilität der Rötungsanalyse des „AllergoEye" denen eines Menschen bei weitem überlegen.

Schlussfolgerung: Die Plattform „AllergoEye“ in Kombination mit KPT, ist zur Diagnose und Kontrolle der Hyposensibilisierung einer allergischen Rhinokonjunktivitis und als quantitative Methode für klinische Studien in der Allergologie bestens geeignet.

\section{P1.7}

\section{Doppelexposition mit Ozon und Gräserpollen} in der Allergen-Expositionskammer bei Patienten mit allergischer Rhinokonjunktivitis: Eine prospektive Fall-Kontroll-Studie

\section{J. Fluhr, P. Joshi, K.-C. Bergmann, T. Zuberbier}

Klinik für Dermatologie, Venerologie und Allergologie, Charité - Universitätsmedizin Berlin, Berlin, Deutschland

Hintergrund: Durch die dramatischen Änderungen der klimatischen Bedingungen wird eine Zunahme von allergischen Symptomen sowie eine Zunahme an Pollenallergikern berichtet. Darüber hinaus ist eine Symptomverstärkung bei Ozonbelastung zu beobachten. Es scheint einen Zusammenhang zwi- 
schen Allergien und einer negativen Beeinflussung epithelialer Barrierefunktionen zu geben.

Ziel: Ziel der Studie war es, die Wirkung von Ozon und Pollen in einer kontrollierten Exposition zu quantifizieren. Es sollte die Hypothese überprüft werden, ob Ozon eine Zunahme der mukosalen-, und Haut-Symptome unter Pollenexposition bei Gräserpollenallergikern induziert.

Methodik: Die prospektive Fall-Kontroll-Studie erfolgte mit 8 Gräserpollenallergikern und 8 Nichtallergikern. Die Exposition erfolgte in der Pollenkammer mit Gräserpollen sowie Ozon. Wir verglichen eine Doppelexposition (DE) mit kürzerer und längerer Dauer. Dokumentiert wurden das generelle Wohlbefinden der Probanden, bronchiale und nasale Symptome. Hautphysiologische Parameter wurde anhand der Hornschichtfeuchtigkeit, Hautrötung, pH-Wert und Barrierefunktion bestimmt.

Ergebnisse: Wir konnten zeigen, dass eine DE bei Pollenallergikern im Vergleich zu gesunden Probanden zu einer signifikanten Induktion der nasalen Sekretion führt, die bei längerer Expositionsdauer ausgeprägter war. Das allgemeine Wohlbefinden war unter der DE deutlich gegenüber Pollen bzw. Ozon alleine verschlechtert mit einem negativen Einfluß der DEDauer. Bezüglich der Hautphysiologie führt die DE zu einer Verschlechterung der Barrierefunktion und Hornschichtfeuchtigkeit, einem Anstieg des pH-Wertes sowie einer Zunahme der Hautrötung.

Diskussion: Die Nasenschleimhaut wird durch die DE mit Ozon und Gräserpollen negativ beeinflußt. Eine kutane Barriereschädigung konnte bei Allergiepatienten mit einer DosisWirkungsrelation nachgewiesen werden. Der Oberflächen-pH wird kann als möglicher Modulationsmechanismus angesehen werden.

\section{ALLERGEN IMMUNOTHERAPY}

\section{P1.8}

\section{Rückgang der Tage mit Rhinitis Exazerbationen bei Behandlung mit der 300IR Hausstaubmilben- SLIT-Tablette}

\section{O. Pfaar ${ }^{1}$, K.-C. Bergmann², U. Kreuzberg ${ }^{3}$, J. Kleine- Tebbe $^{4}$}

${ }^{1}$ Abteilung für Rhinologie und Allergie, Klinik für HalsNasen-Ohren-Heilkunde, Kopf- und Halschirurgie, Universitätsklinikum Marburg, Marburg, Deutschland; ${ }^{2}$ Allergologie und Immunologie, Klinik für Dermatologie und Allergie, Charité, Universitätsmedizin, Berlin, Deutschland; ${ }^{3}$ Stallergenes $\mathrm{GmbH}$, Medical Affairs, Kamp-Lintfort, Deutschland;

${ }^{4}$ Allergie und Asthma Zentrum Westend, Berlin, Deutschland

Hintergrund: Die Allergen-Immuntherapie (AIT) führt bei allergischer Rhinitis (AR) u.a. zur Abnahme der Symptomschwere, was sich auch im Rückgang der AR-Exazerbationen zeigen kann. Der Anteil der Tage mit AR-Exazerbationen bei Patienten mit Hausstaubmilben (HSM)-AR, die mit 300IR HSM-Tabletten behandelt wurden, wurde in zwei randomisierten DBPC-Phase-II und III-Studien untersucht (Demoly et al., EAACI 2021)

Methoden: Patienten mit HSM-AR erhielten ein Jahr lang einmal täglich die 300IR HSM-Tablette oder Placebo. Teilneh- mer bewerteten täglich den Schweregrad ihrer AR-Symptome. Ein Tag mit AR-Exazerbation wurde als ein Tag gewertet, an dem ein Patient zur für die Aufnahme in die Studie erforderlichen Symptomschwere zurückkehrte, d.h. ein täglicher RhinitisGesamtsymptom-Score von mind. 6 oder 5, mit mind. einem der 4 Symptome als schwer eingestuft.

Ergebnisse: In Studie 1 waren 711 (300IR HSM Tablette) und 765 (Placebo) Patienten zu Studienbeginn sowie 586 (300IR) und 676 (Placebo) Patienten zum Studienende auswertbar. In Studie 2 waren 153 (300IR) und 163 (Placebo) zu Studienbeginn sowie 141 (300IR) und 153 (Placebo) zum Studienende auswertbar. In beiden Studien war der Anteil der Tage mit AR-Exazerbationen in den Behandlungsgruppen zu Studienbeginn ähnlich (Studie-1: ca. $71 \%$, Studie-2: ca. $78 \%$ ) und nahm während des Behandlungsjahres ab. Der Anteil der Tage mit AR-Exazerbationen war in der 300IR-Gruppe (Studie-1: $27 \%$, Studie-2: $15 \%$ ) geringer als in der Placebo-Gruppe (Studie-1: $34 \%$, Studie-2: $20 \%)$ mit statistischer Signifikanz in Studie-1 $(p=0,0003)$. Ein mit der 300IR HSM Tablette behandelter Patient kann von einer Reduktion der Tage mit AR Exazerbation um 25 (Studie-1) bzw. 20 Tage (Studie-2) pro Jahr im Vergleich zu einem mit Placebo behandelten Patienten profitieren.

Schlussfolgerung: In zwei Phase-II/III-Studien war die Behandlung mit 300IR HSM-Tabletten mit einer geringeren Anzahl an Tagen mit AR-Exazerbationen im Vergleich zu Placebo verbunden.

\section{P1.9}

\section{Sicherheit und Verträglichkeit der 300IR Hausstaubmilbentablette aus gepoolten Daten klinischer Studien sowie Daten nach der Markteinführung}

\section{Worm', U. Kreuzberg', K.-C. Bergmann'}

${ }^{1}$ Allergologie und Immunologie, Klinik für Dermatologie und Allergie, Charité, Universitätsmedizin, Berlin, Deutschland; ${ }^{2}$ Stallergenes $\mathrm{GmbH}$, Medical Affairs, Kamp-Lintfort, Deutschland

Hintergrund: Wirksamkeit/Sicherheit der 300IR Hausstaubmilben (HSM) Tablette zur sublingualen Immuntherapie (SLIT) wurden im klinischen Entwicklungsprogramm (CDP) bei allergischer Rhinitis (AR) belegt. Sicherheit/Verträglichkeit der 300IR HSM Tablette wurden durch gepoolte Daten aus klinischen Studien und Post-Marketing Follow-Up (PM-FU) untersucht. (Worm et al., Allergy 2020 (75); S109, 1869)

Methoden: Patienten (5-65 Jahre) mit HSM-AR wurden in eine von 8 DBPC-Studien randomisiert. Unerwünschte Ereignisse im Zusammenhang mit der Behandlung (TEAE) mit der 300 IR Tablette oder Placebo wurden zusammengefasst und in Subgruppen von Erwachsenen/Jugendlichen und Kindern analysiert. Seit der Marktzulassung in Japan (2015) wurden Berichte über Nebenwirkungen (UAW) gesammelt.

Ergebnisse: Im CDP wurden 1583 Erwachsene/Jugendliche und 270 Kinder (5-11 Jahre) mit der 300IR-Tablette behandelt, 1588 bzw. 258 erhielten Placebo. In Studien berichteten $57 \%$ der Erwachsenen/Jugendlichen und $50 \%$ der Kinder TEAEs mit 300IR-Tabletten (mit Placebo: 18\% Erwachsene/Jugendliche, $13 \%$ Kinder). TEAEs entsprachen oft oralen, leichten oder mittelschweren Reaktionen, wurden meist an den ersten Tagen berichtet und nahmen in den nächsten 3 Monaten ab. 3 Erwachsene, 1 Jugendlicher und 1 Kind berichteten schwerwiegende TEAEs, die medikamentös behandelt wurden. 
In $>4$ Jahren PM-FU wurden $>80.000$ Patienten mit 300 IRTabletten behandelt, meist waren berichtete UAW lokale, orale Reaktionen. Wichtige, spezifische Risiken, die in Studien und PM Nachverfolgung berichtet wurden, waren anaphylaktische Reaktionen (10 Fälle), schwere laryngopharyngeale Reaktionen (4 Fälle), eosinophile Ösophagitis (3 Fälle) sowie 4 Berichte über Autoimmunerkrankungen, kein Bericht über einen anaphylaktischen Schock.

Schlussfolgerung: Gepoolte Sicherheitsdaten im CDP zeigten das günstige Sicherheitsprofil der 300IR HSM-Tablette. Die Ergebnisse zur PM-Sicherheit stimmten mit Sicherheitsdaten aus klinischen Studien überein.

\section{P1.10}

\section{Clinical relevance of treatment with 300IR house dust mite SLIT tablet}

\section{O. Pfaar ${ }^{1}$, J. Kleine-Tebbe², P. Demoly ${ }^{3}$, F. Bahbah ${ }^{4}$}

${ }^{1}$ Section of Rhinology and Allergy, Department of Otorhinolaryngology Head and Neck Surgery, University Hospital Marburg, Philipps-Universität, Marburg Germany; ${ }^{2}$ Allergy and Asthma Center Westend, Outpatient Clinic \& Research Center Hanf, Ackermann and Kleine-Tebbe, Berlin, Germany;

${ }^{3}$ Desbret Institute of Public Health (IDESP), INSERMUniversity of Montpellier, University Hospital of Montpellier, Montpellier, France;

${ }^{4}$ Stallergenes Greer, Antony, France

Background: The efficacy of the 300IR house dust mite (HDM) tablet was confirmed in a large, RDBPC Global Phase III trial in adults and adolescents with HDM allergic rhinitis (AR) [Demoly et al. JACI 2021]. The clinical relevance of the treatment effect is illustrated hereafter.

Method: Eligible patients with medically confirmed HDM-AR received the 300IR HDM tablet or placebo once daily for one year. Based on the patient-reported symptom severity and use of rescue medication, the treatment effect was assessed through two balanced combined scores, the EAACI-recommended Average Combined Symptom and Medication Score (aCSMS0-6, pre-defined endpoint) and the Average Total Combined Rhinitis Score (ATCRS0-24, post hoc endpoint) as analysed during the primary period (4 weeks at the end of treatment period) using ANCOVA, in overall study population and in the EU subpopulation.

Results: During the primary period, in 1262 evaluable patients $(300 \mathrm{IR}=586$, placebo $=676)$, both ACSMS0- 6 and ATCRS0-24 were significantly lower in the 300IR group than in the placebo group $(p<0.0001)$. Absolute and relative differences from placebo were -0.26 , CI95\% $[-0.38 ;-0.14],-18.0 \%$ and -1.07 , CI95\% $[-1.35 ;-0.79],-18.4 \%$, respectively. A greater and significant treatment effect of HDM tablets vs. placebo $(p<0.0001)$ was observed in European patients $(300 \mathrm{IR}=384$, placebo=434): ACSMS0- $6=-0.31$, ATCRS0-24 $=-1.27$, with relative differences of $-21.7 \%$ and $-22.1 \%$. The clinical relevance of the balanced score results is demonstrated in that the effect of HDM tablets compared to placebo can be translated into a reduction of at least one severity class in one symptom during the whole year.

Conclusion: The treatment effect of the 300IR HDM tablet in terms of relative reduction from placebo was found consistent whichever the score and the scale size. The resulting improvement of at least one severity class in one symptom during the whole year can be considered clinically meaningful from the patient's perspective.

\section{P1.11}

\section{Untersuchung der therapeutischen und} präventiven Anwendung von Ectoin ${ }^{\circledR}$ Allergy Lozenges bei oropharyngealen allergischen Symptomen

R. Khachouk ${ }^{1}$, U. Pieper-Fürst ${ }^{2}$, C. Kolot ${ }^{3}$,

\section{A. Bilstein ${ }^{3,4}$, C. Acikel ${ }^{2}$, J. Singh ${ }^{2}$, N. Werkhäuser ${ }^{2}$,} R. Mösges ${ }^{1,2}$

${ }^{1}$ Institute of Medical Statistics and Computational Biology, Faculty of Medicine, University of Cologne, Cologne, Germany;

${ }^{2} \mathrm{ClinCompetence} \mathrm{Cologne} \mathrm{GmbH}$, Cologne, Germany; 3bitop AG, Carlo-Schmid-Allee 5, 44263 Dortmund, Germany;

${ }^{4}$ Ursatec GmbH, Tholey, Germany

Hintergrund: In dieser Studie wurde untersucht, in wie weit das CE-registrierte Medizinprodukt Ectoin Allergy Lozenges therapeutische oder präventive Effekte auf oropharyngeale allergische Symptome ausübt.

Methoden: Diese $\S 23 \mathrm{~b}$ MPG-Studie wurde als prospektive, randomisierte, kontrollierte Multicenterstudie durchgeführt und umfasste eine Studienvisite. Die Untersuchung wurde an Patienten durchgeführt, die zur ersten Behandlung einer sublingualen Immuntherapie (SLIT) in die Praxis kamen. Sie erhielten 5 Minuten vor Start (präventiv) bzw. 5 Minuten nach Start (therapeutisch) der SLIT eine Lutschtablette; die Kontrollgruppe erhielt keine Lutschtablette. 30 Minuten nach Start der Therapie wurden die SLIT-induzierten Symptome (Jucken, Schwellung, Irritationen) in Mund, Rachen und Lippen durch die Patienten bewertet.

Ergebnisse: Es wurden 89 Patienten eingeschlossen, von denen 32 Patienten der präventiven, 29 Patienten der therapeutischen und 28 Patienten der Kontrollgruppe randomisiert zugeordnet waren.

Die therapeutische oder präventive Gabe von Ectoin Allergy Lozenges resultierte in signifikanter Reduktion oropharyngealer Symptome. In der präventiven bzw. therapeutischen Gruppe war der Juckreiz im Mund um $66 \%$ bzw. $77 \%$ und der OrganSummenwert (Jucken, Schwellung, Irritationen im Mund) um $48 \%$ bzw. 61 \% niedriger verglichen mit der Kontrollgruppe. Der alle Organe (Mund, Lippen, Rachen) einbeziehende Summenwert war in der präventiven Behandlungsgruppe $41 \%$ und in der therapeutischen Behandlungsgruppe $54 \%$ niedriger als in der Kontrollgruppe. Ein mildes unerwartetes Ereignis (stomatales Missempfinden, therapeutische Behandlungsgruppe) mit möglichem Zusammenhang endete am Tag der Behandlung.

Fazit: Sowohl die präventive als auch die therapeutische Gabe von Ectoin Allergy Lozenges führte zur Reduktion oropharyngealer Allergiesymptome. Ectoin Allergy Lozenges stellt damit eine gut verträgliche, nicht-pharmakologische Alternative zur Behandlung oraler Allergiesymptome dar. 
TAPAS - a long-term non-interventional study using $\mathrm{MCT}^{\circledR}$ (MicroCrystalline Tyrosine) adsorbed allergoids to demonstrate non-inferiority of children compared to adults

\section{R. Mösges ${ }^{1,2}$, M. Gerstlauer ${ }^{3}$, S. Allekotte ${ }^{1}$, E. Raskopf', L. England ${ }^{1}$, K. Birkholz ${ }^{4}$, M. F. Kramer ${ }^{4,5}$ S. Güthoff ${ }^{4}$, J. Raab ${ }^{4}$}

${ }^{1}$ ClinCompetence Cologne $\mathrm{GmbH}$, Cologne, Germany; ${ }^{2}$ Institute of Medical Statistics and Computational Biology, Faculty of Medicine, University of Cologne, Cologne, Germany;

${ }^{3}$ Pediatric Pneumology and Allergology Unit, Medical University of Augsburg, Augsburg, Germany; ${ }^{4}$ Bencard Allergie GmbH, Munich, Germany; ${ }^{5}$ Allergy Therapeutics, Worthing, United Kingdom

Background: It is common practice in allergen immunotherapy to use identical dosing regimens for children and adults. However, there is only limited data available supporting this practice. So the primary objective of this study named TAPAS (Tyrosine Allergoid Paediatric and Adult Study) is to demonstrate non-inferiority of clinical efficacy in children compared to adults by exploring long-term treatment effects using subcutaneously administered glutaraldehyde-modified and microcrystalline tyrosine-adsorbed allergoids (MATA) following a perennial posology.

Methods: This is a long-term, prospective, open-label, multi-centre, non-interventional study aiming to include 320 children and adults. Patients with a history of allergic rhinitis, conjunctivitis and/or mild to moderate bronchial asthma, caused by grass and rye pollen and/or birch, alder and hazel pollen can be included. The study design consists of a treatment phase (3 years) and a follow-up period ( 2 years). Daily symptoms and medication scores will be collected via an electronic diary during all respective pollen seasons over 5 years.

Results: The study started in Q4 2020 and is currently conducted at 28 sites in Germany. Patient entries in the electronic diary allow to compare the total combined symptom medication score, averaged over the respective peak pollen season. In addition, changes in quality of life and symptom control of rhinitis and asthma are assessed using validated questionnaires. Up to May 2021, a total of 176 patients (135 adults, 41 children) have been enrolled in the study, while recruitment is still ongoing.

Conclusion: The efficacy and safety of subcutaneous AIT with MATA will be evaluated for non-inferiority by directly comparing a paediatric with an adult population in a long-term setting and under real life conditions. Those data might add meaningful knowledge for AIT in children in general and support the current practice of using identical dosing regimens in children and adults.

\section{Real-World data of short-course specific immunotherapy (SIT) with modified grass/tree allergens using a microcrystalline tyrosine/MCT $\AA$ \& monophosphoryl lipid A/MPL $®$ adjuvant system}

\section{Stollewerk ${ }^{1}$, M. Niebecker ${ }^{1}$, E. Fuchs ${ }^{1}$, L. England ${ }^{2}$, H. Sahin', S. Allekotte ${ }^{2}$, R. Mösges ${ }^{2,3}$, M. Kramer ${ }^{4,5}$, S. Guethoff ${ }^{4}$, K. Birkholz ${ }^{4}$}

${ }^{1}$ Practice for pulmonary and bronchial medicine, Cologne, Germany; ${ }^{2} \mathrm{ClinCompetence} \mathrm{Cologne} \mathrm{GmbH}$, Cologne, Germany; ${ }^{3}$ Institute of Medical Statistics and Computational Biology, Faculty of Medicine, University of Cologne, Cologne, Germany;

${ }^{4}$ Bencard Allergie GmbH, Munich, Germany;

${ }^{5}$ Allergy Therapeutics, Worthing, United Kingdom

Background: Real-world evidence in allergen immunotherapy (AIT) is needed to analyse the efficacy of AIT under reallife setting and support data from clinical trials. The goal of this study was to obtain real life data using a short-course specific immunotherapy (SIT) with modified grass and/or tree allergens with a modern adjuvant system of $\mathrm{MCT}^{\circ} \& \mathrm{MPL}^{\circ}$. Therefore, this study evaluated the change in allergen-specific IgE levels after 3 -year SIT as well as the change in use of symptomatic medication, allergy symptoms and restriction to work/leisure time.

Method: This retrospective mono-centre non-interventional study analysed tree and/or grass allergic patients (pts) treated with a short-course SIT between 01/2010-12/2019. Allergen-specific IgE was measured using an Enzyme-AllergoSorbent test (EAST). Pts were asked about their allergy symptoms \& restrictions to work/leisure time using a questionnaire.

Results: 113 adult pts were included \& data of 112 pts were analyzed. The majority of pts received SIT against tree pollen $(N=60)$. For these pts, the birch-specific IgE levels were significantly reduced after SIT for the total population and for pts with EAST class $>4$ before SIT $(p<0.005)$. The proportion of pts who did not need symptomatic medication during the pollen season increased from 5.4 to $20.7 \%$. The frequency of use of eye drops, nasal spray, asthma spray, antihistamine \& cortisone tablets decreased substantially after SIT. Pts reported an improvement of symptoms of eyes, nose \& lung $(-3.57,-3.76,-2.89$, scale $01-10)$. More than $70 \%$ of pts noted an improved quality of life after SIT.

Conclusion: This non-interventional study confirms the improvement of allergic symptoms, quality of life \& reduction of medication use after short-course SIT with modified grass and/or tree allergens using a modern adjuvant system of MCT \& MPL under routine practice. In addition, a significant reduction in allergen-specific IgE levels after tree pollen specific SIT was observed. 


\section{Design, safety and efficacy profile of the cucumber mosaic virus-like particle (CuMV) modified by genetic fusion with Ara h2 for peanut allergy treatment}

\section{T. C. Velazquez', M. D. Heath', M. Mohsen ${ }^{2,3}$, M. F. Kramer ${ }^{1,4}$, A. Zeltins ${ }^{5}$, M. F. Bachmann ${ }^{2,6}$}

${ }^{1}$ Allergy Therapeutics Ltd, Worthing, United Kingdom; 2Department of BioMedical Research, Immunology RIA, Inselspital, University of Bern, Bern, Switzerland;

${ }^{3}$ Saiba AG, Pfaeffikon, Switzerland;

${ }^{4}$ Bencard Allergie $\mathrm{GmbH}$, Munich, Germany;

${ }^{5}$ Latvian Biomedical Research and Study Centre, Riga, Latvia;

${ }^{6}$ Nuffield Department of Medicine, Centre for Cellular and Molecular Physiology (CCMP), The Jenner Institute, University of Oxford, Oxford, United Kingdom

Background: Peanut allergy is a frequent disease developing early in life. In most cases peanut allergy does not resolve with age and can become a serious health threat. Studies indicate that the major allergens Ara h1 and Ara h2 are recognized by IgE in more than $95 \%$ of patients. We constructed a new vaccine based on the cucumber mosaic virus-like particles with a tetanus toxin T-cell epitope $\left(\mathrm{CuM}_{\mathrm{VTT}}\right)$ genetically fused with Ara $\mathrm{h} 2$. We compared its performance with $\mathrm{CuM}_{\mathrm{VTT}}$ chemically conjugated with Ara hl.

Method: Design of a vector to obtain the $\mathrm{CuM}_{\mathrm{VTT}}$ gene fused with Ara h2 was followed by expression and purification. In parallel, Ara hl was expressed and chemically conjugated with $\mathrm{CuM}_{\mathrm{VTT}}$ VLPs (virus-like particles). SDS-PAGE analysis, electron-microscopy (EM) and dynamic light scattering (DLS) was performed to verify correct folding and purity. The reactogenicity and immunogenicity of the $\mathrm{CuM}_{\mathrm{VTT}}-\mathrm{Ara} \mathrm{h} 2$ vaccine was assessed in sensitised mice. The data was compared to data from the chemically conjugated $\mathrm{CuM}_{\mathrm{VTT}}$-Ara $\mathrm{h} 1$.

Results: High purity of the vaccines was verified and demonstrated by gel analysis, EM and DLS and identity of both vaccines was confirmed. Reactogenicity studies demonstrated the safety of both vaccines compared to native peanut extract by monitoring temperature changes after i.v. injection in sensitised mice. Antibody titers against VLP-incorporated Ara h2 or Ara hl were higher compared to $\mathrm{CuM}_{\mathrm{VTT}}$ alone. No side effects were observed after immunization with either vaccine.

Conclusion: Genetically fused $\mathrm{CuM}_{\mathrm{VTT}}$-Ara h2 demonstrated comparable results with the chemically coupled $\mathrm{CuM}_{\mathrm{VTT}}$-Ara $\mathrm{h} 1$. Both vaccines were non-reactogenic compared to systemic challenge with the whole extract. Specific induction of IgG antibodies was robust. The VLP technology genetically fused with peanut allergen is a new strategy for peanut allergy vaccination. This vaccine candidate is scheduled to enter clinical studies for therapeutic vaccination of humans with peanut allergy.
Design, safety and efficacy profile of the cucumber mosaic virus-like particle (CuMV) modified by genetic fusion with Fel d1 for cat allergy treatment

\section{T. C. Velazquez', M. D. Heath', M. Mohsen ${ }^{2,3}$ M. F. Kramer, ${ }^{1,4}$ A. Zeltins ${ }^{5}$, M. A. Skinner ${ }^{1}$, M.F. Bachmann ${ }^{2,6}$}

${ }^{1}$ Allergy Therapeutics Ltd, Worthing, United Kingdom; ${ }^{2}$ Department of BioMedical Research, Immunology RIA, Inselspital, University of Bern, Bern, Switzerland;

${ }^{3}$ Saiba AG, Pfaeffikon, Switzerland; ${ }^{4}$ Bencard Allergie GmbH, Munich, Germany; ${ }^{5}$ Latvian Biomedical Research and Study Centre, Riga, Latvia;

${ }^{6}$ Nuffield Department of Medicine, Centre for Cellular and Molecular Physiology (CCMP), The Jenner Institute, University of Oxford, Oxford, United Kingdom

Background: Cat allergy in humans is primarily caused by the major allergen Fel d1. We developed a new therapeutic approach by immunizing mice with Fel dl using either a genetic fusion of Fel dl with the cucumber mosaic virus containing the tetanus toxin universal T-cell epitope $\left(\mathrm{CuM}_{\mathrm{VTT}}\right)$, or this VLP (virus-like particle) chemically conjugated with Fel dl.

Method: Design of a vector to obtain the CuMV gene fused with Fel dl was followed by expression and purification. In parallel, Fel d1 was expressed separately from $\mathrm{CuM}_{\mathrm{VTT}}$ VLPs followed by chemical conjugation. SDS-PAGE analysis, electronmicroscopy (EM) and dynamic light scattering (DLS) was performed to verify correct folding and purity. The reactogenicity and immunogenicity of the $\mathrm{CuM}_{\mathrm{VTT}}$-Fel $\mathrm{dl}$ vaccine was assessed in sensitised $\mathrm{BALB} / \mathrm{c}$ mice. The data was compared to data from the chemically conjugated $\mathrm{CuM}_{\mathrm{VTT}}$-Fel d1.

Results: High purity of $\mathrm{CuM}_{\mathrm{VTT}}-\mathrm{Fel} \mathrm{dl}$ was verified and demonstrated by gel analysis, EM and DLS and identity of both vaccines was confirmed. Reactogencity studies demonstrated the safety of both $\mathrm{CuM}_{\mathrm{VTT}}-\mathrm{Fel} \mathrm{d} 1$ vaccines compared to Fel dl allergen by monitoring temperature changes after i.v. injection in sensitised mice. The specific antibody response in mouse sera showed IgG induction against Fel d1 after 14 days of immunization with $\mathrm{CuM}_{\mathrm{VTT}}$-Fel dl chemically coupled or genetically fused. Systemic allergic reactions were reduced in animal groups vaccinated with $\mathrm{CuM}_{\mathrm{VTT}}$-Fel dl compared to $\mathrm{CuM}_{\mathrm{VTT}}$ group.

Conclusion: Genetically fused $\mathrm{CuM}_{\mathrm{VTT}}$-Fel d 1 demonstrated comparable results with the chemically coupled version. Both vaccines were non-reactogenic compared to systemic allergen challenge. The specific induction of IgG antibodies for Fel d1 was robust and sustained. These results suggest a therapeutic advantage for a Fel dl vaccine and highlight a novel approach in the design and application of VLPs genetically fused with an antigen target. 


\section{Studie zur Sicherheit einer verkürzten Aufdosierung mit einem unmodifizierten Hausstaubmilbenextrakt zur subkutanen Immuntherapie: Design}

\section{Klimek', C. Vogelberg ${ }^{2}$, C. Willers ${ }^{3}$, K. Duwensee ${ }^{3}$, C. Praechter ${ }^{3}$, M. Jutel ${ }^{4,5}$}

'Zentrum für Rhinologie und Allergologie, Wiesbaden, Deutschland;

${ }^{2}$ Fachbereich Kinderpneumologie/Allergologie, Universitätsklinikum Carl Gustav Carus, Technische Universität Dresden, Dresden, Deutschland; ${ }^{3}$ Allergopharma GmbH \& Co. KG, Reinbek, Deutschland; ${ }^{4}$ All-MED Medical Research Institute, Wroclaw, Poland; ${ }^{5}$ Department of Clinical Immunology, Wroclaw Medical University, Wroclaw, Polen

Hintergrund: Die subkutane Immuntherapie (SCIT) mit einem seit 1992 in Deutschland zugelassenen unmodifizierten Hausstaubmilbenextrakt ist effektiv und sicher. Die Standardaufdosierung umfasst 14 wöchentliche Injektionen, was für die Patienten zeitaufwändig und wenig komfortabel erscheint.

Ziel: Sicherheit und Verträglichkeit einer verkürzten Aufdosierung aus 6 Injektionen der Stärke 3 werden mit der Standardaufdosierung verglichen.

Methode: Diese multizentrische, randomisierte, offene Phase II-Studie wird in Deutschland und Polen durchgeführt. Patienten beiderlei Geschlechts zwischen 12 und 65 Jahren werden eingeschlossen, wenn sie einen positiven Hautpricktest und spezifisches IgE $(\geq 0,7 \mathrm{kU} / \mathrm{l})$ auf $D$. pteronyssinus und/oder D. farinae sowie in der Anamnese eine moderate bis schwere allergische Rhinitis oder Rhinokonjunktivitis auf Hausstaubmilben (HSM) seit mindestens 2 Jahren mit oder ohne Asthma aufweisen. Für das unmodifizierte, Aluminiumhydroxid-adsorbierte HSM-SCIT-Präparat wird die Aufdosierung mit dem verkürzten Schema (6 Injektionen, Stärke 3) mit der des Standardschemas (14 Injektionen, Stärken 1 bis 3) verglichen. Alle Patienten erhalten anschließend 2 zusätzliche Erhaltungsdosen. Alle unerwünschten Ereignisse werden dokumentiert, die Verträglichkeit wird von Ärzten und Patienten auf einer 5-stufigen Likert-Skala beurteilt.

Schlussfolgerung: Ein verkürztes Schema zu Aufdosierung reduziert die Anzahl der Besuche in der ärztlichen Praxis und macht die SCIT für den Patienten komfortabler. Dies könnte einen Einfluss auf die Adhärenz der Patienten haben, die für die (Langzeit-)Effekte von großer Bedeutung ist. Zusätzlich reduziert die Verwendung einer Stärke das Risiko der Verwechslung von Vials verschiedener Stärken während der Aufdosierung. Somit würde ein modernes, sicheres, verträgliches und praktisches Aufdosierungsschema mit einem etablierten SCIT-Präparat zur Verfügung stehen.
Verträglichkeit einer sublingualen

Immuntherapie in Tropfenform bei Patienten mit Frühblüherallergie in der täglichen Routine nach der Zulassung

\section{Owenier, H. van Schijndel, D.i Yu, M. Verhagen, M. Leineweber, A. Distler}

${ }^{1} \mathrm{HAL}$ Allergie GmbH, Düsseldorf, Deutschland;

${ }^{2} \mathrm{HAL}$ Allergy BV, Leiden, Niederlande

Hintergrund: In dieser prospektiven, nicht-interventionellen Studie (NIS) wurde die Verträglichkeit der sublingualen Birken- bzw. Baumpollen-Allergenpräparate (HAL Allergy BV, Leiden, Niederlande) in der täglichen Praxis über 8 Monate (M) untersucht. Die Präparate wurden im Rahmen der Therapieallergene-Verordnung in 2018 zugelassen.

Methoden: Insgesamt nahmen 84 Arztpraxen in Deutschland teil. Eingeschlossene Patienten (Pat) erhielten entweder einen Allergenextrakt aus Birkenpollen (BiP) oder eine Mischung aus Birken-, Hasel- und Erlenpollen (MB). Auftretende Nebenwirkungen (NW) und die Compliance wurden über einen elektronischen Dokumentationsbogen (eCRF) von den teilnehmenden Ärzten erfasst. Zur Erfassung fanden insgesamt 5 Arztbesuche in den $8 \mathrm{M}$ statt.

Ergebnisse: Daten von 432 Pat waren auswertbar. 208 Pat erhielten BiP und 224 Pat bekamen MB. Davon erreichten $96 \%$ der Pat die Höchstdosis von 5 Tropfen. Der primäre Studienparameter war die Verträglichkeit beider Präparate. Hinsichtlich lokaler und systemischer Reaktionen (Rk) zeigte sich folgendes Bild:

\section{Allergen Lokale Rk $n$ (\%) Systemische Rk $n$ (\%)}

BiP $(N=$ 208) $47(22,6) 9(4,3)$

MB $(N=224) 49(21,9) 18(8,0)$

Total $(N=432) 96(22,2) 27(6,3)$

Schwere systemische Rk Grad IV traten nicht auf. Die Intensität lokaler Rk wurde in den meisten Fällen mit mild bis moderat angegeben. Die meisten NW traten innerhalb von 30 Minuten nach der Einnahme auf. Die Mehrzahl der NW wurde während der Grundbehandlung dokumentiert. 332 Pat nahmen die Tropfen bis zum Ende der $8 \mathrm{M}$ ein. Die Pat Compliance betrug somit $71 \%$. Mit der Therapie zufrieden bzw. sehr zufrieden waren 98,6\% der Pat.

Zusammenfassung: Insgesamt konnten $96 \%$ der Pat die Höchstdosis von 5 Tropfen erreichen. Die Verträglichkeit beider Präparate war ähnlich und bestätigt die Daten der zuvor durchgeführten Phase-III-Studie.

\section{P3.8}

Status der klinische Nachzulassungsstudien im Rahmen der Therapieallergene-Verordnung (TAV): Update der systematischen Recherche

\section{A. Horn', C. Bachert ${ }^{2}$, D. Brehmer ${ }^{3}$}

${ }^{1} \mathrm{HNO}-$ Praxis am Neckar, Heidelberg, Deutschland; ${ }^{2} \mathrm{HNO}-$ Universitätsklinik, Gent, Belgien; ${ }^{3} \mathrm{HNO}-$ Praxis Alte Post, Göttingen, Fakultät für Medizin, Universität Witten/Herdecke, Witten, Deutschland

Hintergrund: Im Rahmen der Therapieallergene-Verordnung (TAV) gilt seit 2008 die Zulassungspflicht für die häufig 
vorkommenden Allergene (Gräser, Bäume, Milben). Eine per review Publikation (Horn, Bachert, Brehmer, ZEFQ 2021) stellte fest, dass in >12 Jahren TAV bisher lediglich ein Studienprogramm zu zwei Zulassungen führte. Bei weiteren Präparaten im TAV-Prozess kann nicht mit einer zeitnahen Zulassung gerechnet werden. Die vorliegende Arbeit prüft, ob die Ergebnisse der Übersichtsarbeit noch Bestand haben oder ob es neue Aktivitäten im Rahmen der TAV gibt.

Methodisches Vorgehen: Die Methodik dieser Arbeit baut analog zur Übersichtsarbeit auf der systematischen Durchsuchung der klinischen Studienregister (clinicaltrialsregister.eu \& clinicaltrials.gov) auf. Dabei umfassen die Suchbegriffe die Indikation, die Spezifikation der Hauptallergene sowie die relevanten Unternehmen u. Produkte.

Ergebnisse: Es befinden sich, nach Zuordnung zu den homologen Gruppen, weiterhin 32 Präparate im TAV-Prozess. Im Vergleich zur Übersichtsarbeit haben drei weitere Präparate (bisher ohne Studienaktivität) eine $\mathrm{Ph}$. II-Studie gemeldet, wobei nicht eindeutig ist, ob die Studien gestartet sind. Für 14 Präparate ( $44 \%$ ) lässt sich in den analysierten Studienregistern keine TAV-relevante Studienaktivität erkennen. Bei den 18 Präparaten, für die Studienaktivitäten sichtbar sind, kann auf Basis bisher einsehbarer Studienergebnisse unverändert nicht mit einer zeitnahen Zulassung gerechnet werden. Eine zum Stand der vorherigen Arbeit noch nicht veröffentlichte Ph. III-Studie konnte ihren primären Endpunkt nicht erreichen.

Diskussion: Aktuell lässt sich weiterhin kein Präparat identifizieren, das kurz vor der Zulassung im Rahmen der TAV steht. Dies berücksichtigend erneuern die Autoren mit Blick auf die Marktverfügbarkeit zugelassener Präparate für alle Hauptallergene in beiden Applikationen ihre Empfehlung zum Einsatz bereits zugelassener Therapieallergene analog der Arzneimittel-Rahmenvorgabe.

\section{P3.9}

\section{Patientenbefragung zu Bedürfnissen und} Erwartungen an eine Hyposensibilisierung in einer HNO-Praxis in Falkensee, Brandenburg

\section{A. Nöding ${ }^{1}$, C. Dietrich ${ }^{1}$, A. Klein ${ }^{2}$}

${ }^{1} \mathrm{HNO}$ Praxis Falkensee, Brandenburg, Deutschland; ${ }^{2}$ Bencard Allergie GmbH, Market Access \& Scientific Affairs, München, Deutschland

Hintergrund: Grundlage einer erfolgreichen Therapie im Rahmen der allergenspezifischen Immuntherapie (SIT) ist die Berücksichtigung der individuellen Bedürfnisse des Patienten. Ebenso Faktoren, die es dem Patienten erschweren, die Therapie erfolgreich abzuschließen. Deren Berücksichtigung in der Phase der Therapieauswahl kann die Adhärenz positiv beeinflussen [Sondermann et al. Allergologie. 2011; 9: 441-446].

Zielsetzung: Ziel der vorliegenden Untersuchung war es, die Bedürfnisse und Erwartungen des Patienten einer SIT mittels Befragung zu erfassen und zu analysieren.

Methode: Die Befragung bezog sich auf den individuellen Aufwand zur Durchführung der Therapie einschließlich der Erwartungen zum Verlauf. Dokumentiert wurden die Antworten auf dem Patienten Benefit Index (PBI)-Fragebogen ${ }^{2}$. Auf diesem konnten die Patienten Ihren individuellen Aufwand auf einer Skala von 0 „unwichtig, gering“ bis 4 „sehr wichtig, hoch“ einschätzen.

Ergebnisse: 242 Patienten, in einem Alter von 16-69 Jahren, haben den Fragebogen vollständig ausgefüllt. 198 (82\%) der Befragten bewerten Ihren persönlichen Aufwand für einen Pra- xisbesuch von 2-4, davon 60 (25\%) mit hoch. Die Frage, ob Sie sich zutrauen über 3 Jahre ein Präparat täglich (z. B. Tabletten, Tropfen) einzunehmen, beantworten 149 (62\%) mit nein. Eine enge Betreuung im Therapieverlauf inklusive Erinnerungen ist für $78 \%$ der befragten Patienten sehr wichtig.

Schlussfolgerungen: Es konnte gezeigt werden, dass nur ein Viertel der Patienten den Aufwand für einen persönlichen Praxisbesuch im Rahmen einer SIT als „hoch“ einschätzt. Dagegen gaben $62 \%$ an, dass eine tägliche Therapie für Sie nicht vorstellbar wäre. Die Berücksichtigung solcher Angaben kann die Adhärenz von Patienten unterstützen [Index zu Nutzen und Bedürfnissen in der Therapie, Augustin 2006].

\section{P3.10}

TARGET - Impact von zugelassenen

MicroCrystalline Tyrosine (MCT®) - adsorbierten Pollen SCIT Allergoiden auf die allergische Rhinitis (AR) und Asthma unter Real-Life-Bedingungen

\section{S. Becker ${ }^{1}$, L. Klimek², S. Kruppert ${ }^{3}$, C. Vogelberg ${ }^{4}$}

${ }^{1}$ Hals-, Nasen-, Ohrenklinik und Poliklinik, Universitätsklinik Tübingen, Deutschland;

'Zentrum für Rhinologie und Allergologie, Wiesbaden, Deutschland;

${ }^{3} \mathrm{Q}$ VIA Commercial GmbH \& Co. OHG, Frankfurt am Main, Deutschland;

${ }^{4}$ Universitätsklinikum Carl Gustav Carus, Dresden,

Deutschland

Hintergrund: Die subkutane Immuntherapie (SCIT) mit Allergoiden verfügt über ein günstiges Sicherheitsprofil. Die Wirksamkeit wurde in DBPC Studien und Metaanalysen für diverse Produkte und MCT $^{\oplus}$-adsorbierte SCIT Allergoide unter idealen klinischen Studienvoraussetzungen nachgewiesen. Primäres Ziel der „Tyrosine-Allergoid - Real World Evidence in Germany - Effectiveness in AIT“ (TARGET) Studie ist der Nachweis therapeutischer Effekte eines zugelassenen MCT ${ }^{\ominus}$-adsorbierten Pollen SCIT Allergoids im klinischen Praxisalltag (sog. Effectiveness).

Methode: Durch eine retrospektive Kohortenanalyse sollten Effekte von drei in Deutschland zugelassenen Pollen SCIT Allergoiden (AIT Gruppe) auf die Allergische Rhinitis (AR) und Asthma vs. gematchten Kontrollgruppen (ohne AIT) nachgewiesen werden. AIT Patienten (5-65 Jahre) mit mindestens vier Verordnungen innerhalb von drei Jahren ab Index Datum und mindestens zwei Jahren Follow-Up (nach Ablauf der letzten AIT Verordnung bis Ende der Datenbank im Juni 2020) wurden in die Studie eingeschlossen. Basis der Studie waren longitudinale Verordnungsdaten (IMS LRx ${ }^{\star}$ Datenbank, IQVIA, Deutschland) unter Berücksichtigung eingelöster GKV-Rezepte. Anhand Verordnungen symptomatischer AR- und Asthmamedikation wurde die AR und Asthma Progression ermittelt.

Ergebnisse: In der Index Periode (09/2009-08/2013) erhielten 181.496 Patienten Verordnungen innerhalb der AIT Gruppe, wovon 5959 Patienten die Einschlusskriterien erfüllten. Statistisch signifikant mehr Patienten, die mit $\mathrm{MCT}^{\circ}$-adsorbierten SCIT Allergoiden therapiert wurden, blieben in der FollowUp Periode im Vergleich zur Kontrollgruppe frei von AR- und Asthmamedikation. Diese Effekte wurden für die gesamte AIT Gruppe nachgewiesen.

Schlussfolgerung: Die Ergebnisse der TARGET Studie zeigten den Langzeitnutzen und die Effectiveness zugelassener Pollen SCIT Allergoide einschließlich MCT $^{\circ}$-adsorbierter Allergoide auf die AR und Asthma im Behandlungsalltag. 


\section{ALLERGIC OCCUPATIONAL DISEASES}

\section{P2.4}

\section{Mycobacterium immunogenum - ein weiterer Baustein im Repertoire der Kühlschmierstoff (KSS)-Antigene bei Verdacht auf eine exogen allergische Alveolitis (EAA)}

\section{S. Kespohl', I. Warfolomeow ${ }^{2}$, A. Kolk ${ }^{3}$, S. Maryska ${ }^{1}$, U. Meurer ${ }^{1}$, P. Wattrodt ${ }^{3}$, T. Brüning ${ }^{1}$, R. Merget ${ }^{1}$, M. Raulf}

${ }^{1}$ Institut für Prävention und Arbeitsmedizin der Deutschen Gesetzlichen Unfallversicherung, Institut der Ruhr-Universität Bochum (IPA), Bochum, Deutschland;

${ }^{2}$ Berufsgenossenschaft Holz und Metall (BGHM), Mainz, Deutschland;

${ }^{3}$ Institut für Arbeitsschutz und Arbeitssicherheit der Deutschen Gesetzlichen Unfallversicherung (IFA), St. Augustin, Deutschland

Insbesondere bakterielle Antigene verschiedener Pseudomonaden- und Mykobakterien-Arten sind als klinisch-relevante Auslöser einer exogen allergischen Alveolitis (EAA) durch kontaminierte Kühlschmierstoffe (KSS) beschrieben. Bei RoutineUntersuchungen werden Mykobakterien eher selten identifiziert, da sie selektive Kultivierungsbedingungen benötigen. Dadurch sind sie als potenzielle Auslöser einer KSS-EAA möglicherweise unterrepräsentiert. Um die klinische Relevanz zu untersuchen, wurden 3 Bakterienisolate von Mycobacterium immunogenum kultiviert, das Bakterienmaterial chemisch und thermisch inaktiviert und mittels Precellys und Ultraschall extrahiert. Der Proteinextrakt wurde für die IgG-Testung an ImmunoCAPs gekoppelt. Die serologische Untersuchung mit $M$. immunogenum-Antigen und weiteren, bereits etablierten KSS-Antigenen erfolgte an 17 KSS-EAA-Verdachtsfällen und einem Referenzkollektiv. Ein KSS-Antigen Mix aus drei Arbeitsplatzproben eines Betriebs wurde als Screening-Tool genutzt und in 11 der 17 Verdachtsfälle konnte damit erhöhte IgGKonzentrationen gemessen werden. Weitere IgG-Testungen mit Antigenen einzelne Bakterienisolate zeigten am häufigsten mit $77 \%$ erhöhte IgG-Konzentrationen auf $M$. immunogenum, gefolgt von Pseudomonas oleovorans und Pseudomonas spec (jeweils $65 \%$ ), Pseudomonas alcaliphila (59\%) und Paenibacillus glucanolyticus (29\%). Durch diese erweiterte Testung konnten in sechs Verdachtsfällen, die zuvor im KSS-Antigen Mix unauffällig oder grenzwertig waren $M$. immunogenum als dominantes IgG-Antigen bestimmt werden, davon war $M$. immunogenum in zwei Fällen auch das einzig erhöhte bakterielle Antigen. Pseudomonaden-Antigene waren in acht Verdachtsfällen dominante Antigene, davon auch in einem Fall das einzig erhöhte. Insgesamt konnte durch das erweiterte Testpanel in $82 \%$ der Verdachtsfälle eine dominantes Bakterien-Antigen benannt werden und die Diagnose einer KSS-induzierten EAA unterstützt werden.

\section{P2.5}

Nutzen der Messung des exhalierten

Stickstoffmonoxids vor und

nach dem arbeitsplatzbezogenen Inhalationstest

bei Personen mit Verdacht auf allergisches

Berufsasthma

\section{V. van Kampen, J. Engel, T. Brüning, R. Merget}

Institut für Prävention und Arbeitsmedizin der Deutschen Gesetzlichen Unfallversicherung, Institut der RuhrUniversität-Bochum (IPA), Bochum, Deutschland

Es ist bekannt, dass es nach einem arbeitsplatzbezogenen Inhalationstest mit Allergenen (AIT) zu einem Anstieg des fraktionierten exhalierten Stickstoffmonoxids (FeNO) kommen kann. Ziel der Studie war es, die diagnostische Wertigkeit von FeNOMessungen vor und nach dem AIT bei Personen mit Verdacht auf Berufsasthma zu beurteilen. 148 Patienten mit Verdacht auf Berufsasthma unterzogen sich einem AIT mit den vermuteten Allergenen. Anhand der Standard-Lungenfunktionskriterien $\left(\mathrm{FEV}_{1}\right.$, sRt) wurde der AIT als positiv, negativ bzw. fraglich bewertet. Die FeNO-Messungen erfolgten vor und $24 \mathrm{~h}$ nach dem AIT. Mit den AIT-Bewertungen (pos./neg.) als Gold-Standard wurden Receiver-Operating-Characteristic (ROC)-Kurven berechnet. Für Personen mit einem negativen oder fraglichen AIT-Ergebnis, aber einer FeNO-Erhöhung nach dem AIT, wurde unter Berücksichtigung der FeNO-Werte und zusätzlicher Parameter (z. B. Veränderung der bronchialen Hyperreaktivität oder des Eosinophilenanteils im Sputum über den AIT) eine individuelle Expertenbewertung durchgeführt. Anhand der Lungenfunktion wurde der AIT für 31 Personen $(21 \%)$ positiv, für 105 (71\%) negativ und für $12(8 \%)$ fraglich eingestuft. Mit dieser AIT-Bewertung als Gold-Standard ergab die ROC-Analyse bei der geforderten Spezifität von 0,9 einen FeNO-Anstieg $\geq 13 \mathrm{ppb}$ als Cut-off (Sensitivität: 0,5). Insgesamt 17 Personen mit negativem oder fraglichem AIT-Ergebnis zeigten einen FeNO-Anstieg $\geq 13 \mathrm{ppb}$, von denen nach der Expertenbewertung 10 Personen die Diagnose „Berufsasthma“ und 3 ,wahrscheinliches Berufsasthma" erhielten. Somit konnte bei mindestens 8,5\% der 117 Personen mit negativem oder fraglichem AIT eine falsch-negative Diagnose verhindert werden. Ein Anstieg des FeNO während des AIT von $\geq 13 \mathrm{ppb}$ war in unserem Kollektiv stark prädiktiv für Berufsasthma. Aus diesem Grunde sollte das Ergebnis der FeNO-Messung vor und nach AIT als ein zusätzliches Kriterium bei der Beurteilung des AIT und für die Diagnosestellung herangezogen werden. 


\section{Allergene in permanenten Tätowierfarben - erste Ergebnisse des Informationsverbundes Dermatologischer Kliniken (IVDK)}

\section{S. Schubert ${ }^{1}$, M. Dirks ${ }^{2}$, H. Dickel ${ }^{3}$, C. Lang ${ }^{4}$, J. Geier $^{1}$}

${ }^{1}$ Informationsverbund Dermatologischer Kliniken (IVDK), Institut an der Universitätsmedizin Göttingen, Göttingen, Deutschland;

${ }^{2}$ THE 3 PYLONS GmbH, Sankt Martin an der Raab, Österreich;

${ }^{3}$ Klinik für Dermatologie, Venerologie und Allergologie, St. Josef-Hospital, Universitätsklinikum der Ruhr-Universität Bochum (UK RUB), Bochum, Deutschland;

${ }^{4}$ Klinik für Dermatologie, Universitätsspital Zürich, Zürich, Schweiz

Hintergrund: Permanente Tätowierungen und Permanent Make-up (im Folgenden „Tattoos“ genannt) sind auch bei dermatologischen Patienten sehr beliebt.

Patienten und Methoden: Zwischen 08/2018 und 07/2020 wurde die neue Epikutantestempfehlung der Deutschen Kontaktallergie Gruppe (DKG) zur Diagnostik von nichtinfektiösen Tätowiermittel-Unverträglichkeitsreaktionen bei 57 Patienten (40 Frauen, 17 Männer) in 23 von 58 Dermatologischen Zentren des IVDK angewendet. Die gesamte Empfehlung umfasst vier etablierte DKG-Testreihen (Standardreihe, Leder- und Textilfarben, Industrielle Biozide, Konservierungsmittel in Externa) und die neue DKG-Testreihe „Tätowiermittel“. Bei $n=35$ (61,4\%) dieser Patienten bestand der Verdacht auf eine Kontaktallergie, $n=16(28,1 \%)$ wurden zum Ausschluss getestet.

Ergebnisse: An Tag 3 wurden 69 positive Reaktionen auf 27 Testpräparationen dokumentiert [+: $41(59,4 \%),++: 15(21,7 \%)$, +++: $13(18,9 \%)]$. Die häufigsten Reaktionen wurden auf Nickelsulfat ( $n=12 ; 25 \%), p$-Phenylendiamin (PPD, $n=7 ; 15 \%$ ), and Kobaltchlorid $(n=6 ; 12 \%)$ beobachtet. Außerdem wurden weitere 8 Konservierungsmittel, 3 Metalle und 3 Bindemittel positiv getestet.

Diskussion: Fünf der PPD-positiven Patienten wurden durch schwarzes Henna sensibilisiert, reagierten extrem stark (+++) und zusätzlich auf Dispers-Farbstoffe. Zwei Patienten mit Komplikationen in roten Tattoos und schwach positiver Reaktion (+) auf PPD. Die klinische Relevanz von positiven Epikutantestreaktionen ist oft nicht zu ermitteln. Metalle sind als Verunreinigungen nie auf Tattoo-Farben deklariert. Im Einzelfall bleibt oft unklar, ob eine Nickel- oder Kobalt-Sensibilisierung relevant für eine Tattoo-Reaktion ist oder ob eine gewisse BodyArt-Affinität der Patienten zur Sensibilisierung durch beispielsweise Piercings oder Modeschmuck geführt hat.

Ausblick: Im IVDK läuft eine Tattoo-Studie mit erweiterter Körperschmuckanamnese und der Möglichkeit zur Pigmentanalyse in Hautproben (http://ivdk.org/de/aktivitäten/tattoostudie/).

\section{Beruflich bedingte Kontaktekzeme bei Malern und Lackierern: Daten des Informationsverbundes Dermatologischer Kliniken (IVDK), 2000 bis 2019}

\author{
S. Schubert ${ }^{1,2}$, A. Bauer ${ }^{3}$, U.Hillen ${ }^{4}$, T. Werfel ${ }^{5}$, \\ J. Geier ${ }^{1,2}$, R. Brans ${ }^{6,7,8}$
}

${ }^{1}$ Informationsverbund Dermatologischer Kliniken (IVDK), Institut an der Universitätsmedizin Göttingen, Göttingen, Deutschland;

${ }^{2}$ Niedersächsisches Institut für Berufsdermatologie (NIB), Göttingen, Deutschland;

${ }^{3}$ Klinik und Poliklinik für Dermatologie, Universitäts

AllergieCentrum, Universitätsklinikum Carl Gustav Carus,

Technische Universität Dresden, Dresden, Deutschland; ${ }^{4}$ Klinik für Dermatologie und Venerologie, Vivantes Klinikum Neukölln, Berlin, Deutschland;

${ }^{5}$ Abteilung Immunodermatologie und Allergieforschung, Klinik für Dermatologie, Allergologie und Venerologie, Medizinische Hochschule Hannover, Hannover, Deutschland;

${ }^{6}$ Niedersächsisches Institut für Berufsdermatologie (NIB), Osnabrück, Deutschland;

${ }^{7}$ Institut für interdisziplinäre Dermatologische Prävention und Rehabilitation (iDerm), Universität Osnabrück, Osnabrück, Deutschland;

${ }^{8}$ Abteilung Dermatologie, Umweltmedizin und Gesundheitstheorie, Universität Osnabrück, Osnabrück, Deutschland

Hintergrund: Aufgrund ihrer beruflichen Exposition gegenüber Kontaktallergenen und Irritanzien haben Maler und Lackierer ein erhöhtes Risiko für beruflich bedingte Kontaktekzeme.

Zielsetzung: Ziel war es, das Spektrum berufsbedingter Sensibilisierungen bei Malern und Lackierern zu beschreiben und die aktuelle Epikutantestempfehlung der Deutschen Kontaktallergie-Gruppe (DKG) zu überprüfen.

Patienten und Methoden: IVDK-Daten aus den Jahren 2000 bis 2019 wurden retrospektiv analysiert. Für vergleichende Analysen wurden alters- und geschlechtskonforme Patientengruppen definiert: Studiengruppe - männliche Maler und Lackierer mit Berufsdermatose im Alter von 20-59 Jahren $(n=557)$, Kontrollgruppe I - altersgleiche männliche Maler und Lackierer ohne Berufsdermatose ( $n=422)$, Kontrollgruppe II - altersgleiche männliche Patienten mit Berufsdermatose, die nie als Maler und Lackierer gearbeitet haben $(n=13.862)$.

Ergebnisse: Maler und Lackierer mit Berufsdermatose haben im Vergleich zu Patienten mit Berufsdermatose, die in anderen Berufen arbeiten, signifikant häufiger allergische Kontaktekzeme ( $42,5 \%$ vs. $32,1 \%)$, Gesichtsekzeme (13,6\% vs. $5,2 \%$ ) und aerogene Ekzeme (4,1\% vs. 1,2\%). Positive Epikutantestreaktionen auf Epoxidharz $(17,7 \%)$, Methylisothiazolinon (MI) (21,8\%) und (Chlor-)Methylisothiazolinon (MCI/MI) $(12,0 \%)$ waren bei Malern und Lackierern mit Berufsdermatose signifikant häufiger als in beiden Kontrollgruppen. EpoxidharzSensibilisierungen waren in der Studiengruppe signifikant mit Gesichtsekzemen und aerogenen Ekzemen assoziiert. In der Testreihe „DKG industrielle Biozide“ waren Benzisothiazolinon $(6,8 \%)$ und Octylisothiazolinon $(6,3 \%)$ am häufigsten positiv.

Fazit: Epoxidharz, MI und MCI/MI stellen die wichtigsten beruflichen Kontaktallergene bei Malern und Lackierern dar. Bei Verdacht auf eine Berufsdermatose sollten neben der DKG Standardreihe, die DKG-Testreihen „Kunstharze und Kleber“ und „industrielle Biozide“ getestet werden. 
P2.9

\section{Süßkartoffelallergie als Berufskrankheit - Rhinitis, Asthma und Kontakturtikaria in einer Bearbeitungsstätte für Wurzelgemüse}

\section{S. Jungewelter}

Finnish Institute of Occupational Health, Helsinki, Finnland

Die Süßkartoffel (Ipomoea batatas) ist eine Nutzpflanze, deren unterirdischen Speicherwurzeln als Nahrungsmittel genutzt werden. Ursprünglich stammt sie aus Südamerika. Mit einer Jahresernte von etwa 92 Millionen Tonnen (Stand 2018) befindet sie sich - nach Kartoffeln und Maniok - auf dem dritten Platz der Weltproduktion von Wurzel- und Knollennahrungspflanzen. Der Anbau in Deutschland nimmt zu, ebenso das Importvolumen, das sich in den vergangenen fünf Jahren verdoppelt hat. Wir berichten über eine Patientin mit Typ-I-Sensibilisierung gegenüber Süßkartoffel, die seit 9 Jahren in einer Wurzelgemüsebearbeitungsanlage arbeitete, und sich mit Verdacht auf eine Berufserkrankung in unserer Klinik vorstellte. Die Patientin bearbeitete täglich hunderte Kilos von Süßkartoffeln und Pastinaken, mit Schutzhandschuhen aber ohne Schutzmaske. Sie bekam im 1. Jahr Rhinitis und 6 Jahre später asthmatische Symptome und Urtikaria nach Süßkartoffelkontakt. Spezifische IgE Antikörpertiter gegen Süßkartoffel waren erhöht $(1,4-1,8$ kU/l). Skin Prick Tests waren positiv für Süßkartoffel (9 mm), Pastinake $(5 \mathrm{~mm})$ und Sellerie $(4 \mathrm{~mm})$. Die Hautprovokation war positiv für Süßkartoffel und Pastinake, so konnten wir eine berufliche Kontakturtikaria diagnostizieren. Die Diagnose einer allergischen Rhinitis bestätigte sich durch eine nasale Provokation mit 0,2 $\mathrm{ml} \mathrm{0.5 \%} \mathrm{Süßkartoffelsaft,} \mathrm{beidseits} \mathrm{auf} \mathrm{die}$ Nasenachleimhäute gesprüht. Die Provokation der unteren Atemwege musste unterbrochen werden, da die Patientin Lippen- und Zungenschwellung, Rachenkratzen, Rhinitis, Husten und Kopfschmerzen entwickelte. Die Diagnose eines Berufsasthmas wurde anhand der Allergie und der Symptome beim Bearbeiten der Süßkartoffel gemacht. Berichte über Asthma, Rhinitis oder Urtikaria nach beruflicher Süßkartoffelexposition sind in der Literatur nicht zu finden. Es ist in der Zukunft von einer steigenden Anzahl von Berufserkrankungen auszugehen, wenn die Süßkartoffel vermehrt importiert, angebaut und bearbeitet wird.

\section{P2.10}

Tag 7 Ablesung in der Epikutantestung - never miss a patient?

S. Forkel', S. Schubert ${ }^{2}$, H. Dickel ${ }^{3}$, M. Gina ${ }^{4}$, C. Schröder-Kraft ${ }^{5}$ D. Vieluf 6 , R. Brans ${ }^{7}$, B. Kreft ${ }^{8}$, G. Wurpts' ${ }^{9}$ J. Geier' ${ }^{2}$, T. Buhl' ${ }^{1}$

${ }^{1}$ Klinik für Dermatologie, Venerologie und Allergologie, Universitätsmedizin Göttingen, Göttingen, Deutschland; ${ }^{2}$ Informationsverbund Dermatologischer Kliniken, Institut an der Universitätsmedizin Göttingen, Göttingen, Deutschland;

${ }^{3}$ Klinik für Dermatologie, Venerologie und Allergologie, Ruhr-Universität Bochum, St. Josef-Hospital, Bochum, Deutschland;

${ }^{4}$ Abteilung für berufsbedingte Hauterkrankungen, BG Klinikum Falkenstein, Falkenstein, Deutschland; ${ }^{5}$ Institut für interdisziplinäre Dermatologische Prävention und Rehabilitation, BG Klinikum Hamburg, Hamburg, Deutschland;

${ }^{6}$ Abteilung Allergologie, Berufsdermatologie und Photodermatologie, Dermatologikum Hamburg, Hamburg, Deutschland;

${ }^{7}$ Institut für interdisziplinäre Dermatologische Prävention und Rehabilitation, Universität Osnabrück, Osnabrück, Deutschland;

${ }^{8}$ Klinik für Dermatologie, Venerologie und Allergologie, Universitätsklinikum Halle, Halle, Deutschland; ${ }^{9}$ Klinik für Dermatologie, Venerologie und Allergologie, Universitätsklinikum Aachen, Aachen, Deutschland

Hintergrund: Grundsätzlich wird die Ablesung des Epikutantests an Tag 2 (D2) und zusätzlich an D3 (oder D4) empfohlen. Vorangegangene Studien zeigten, dass durch eine zusätzliche Ablesung an D7 bis zu $15 \%$ mehr positive Reaktionen verzeichnet werden konnten.

Methoden: Es erfolgte eine retrospektive multizentrische Datenauswertung von 4687 Patienten aus den Jahren 20102019. Die Patienten erhielten eine Epikutantestung mit der Standardreihe, und entsprechend der Indikation wurden weitere DKG-Reihen getestet. Es wurden die Ergebnisse der positiven Epikutantestungen mit Hinblick auf erstmalig an D7 positive Reaktionen untersucht. Zusätzlich erfolgte eine Analyse des Crescendoverlaufs der Reaktionen von D3 zu D7.

Ergebnis: In Abhängigkeit der getesteten DKG-Reihe würden ohne eine D7-Ablesung zwischen 4,4-26,8\% der positiven Reaktionen nicht erkannt werden. Die höchste Rate an erstmalig an D7 positiven Reaktionen fand sich in der DKG Standardreihe, DKG Dentalmetalle und DKG Leder und Schuhe. Mit Hinblick auf individuelle Patientencharakteristika konnte festgestellt werden, dass ein Patientenalter über 50 Jahre positiv mit Spätreaktionen korreliert und eine vorliegende Reaktion auf SLS negativ mit Spätreaktionen assoziiert ist. Zusätzlich konnte eine Abhängigkeit der D7 Reaktionen von einer Expositionsdauer von $48 \mathrm{~h}$ festgestellt werden.

Schlussfolgerung: Aus den am häufigsten getesteten Allergenen würden im Mittel 11,7\% der Reaktionen ohne eine D7-Ablesung nicht erfasst werden. Neben den bekannten Allergenen mit Tendenz zu Spätreaktionen nach D3 konnten zahlreiche weitere Allergene identifiziert werden. 


\section{BRONCHIAL ASTHMA}

P4.1

\section{Spektrum der IgE-vermittelten Sensibilisierungen bei Patienten mit schwerem Asthma - das ATLAS- Projekt.}

\section{J. Schreiber ${ }^{1}$, E. Lücke1, Q. Wu' ${ }^{1}$, C. Mailänder ${ }^{2}$}

1Universitätsklinik für Pneumologie, Universitätsklinikum, Otto-von-Guericke-Universität Magdeburg, Deutschland; ${ }^{2}$ Novartis Pharma GmbH, Nürnberg, Deutschland

Einführung: Eine sorgfältige Bewertung der allergischen Sensibilisierung ist für die Behandlung des schweren Asthmas unumgänglich. Die Bedeutung seltenerer Allergene ist noch weitgehend ungeklärt. Das Ziel dieses deutschlandweiten Projektes war es, Daten über den Sensibilisierungsstatus von 55 Allergenen, bei schwerem Asthma zu gewinnen und hinsichtlich des prädiktiven Wertes von anamnestischen Angaben, von Sensibilisierungsclustern, der Bedeutung seltener und wenig bekannter Allergene und im Hinblick auf regionale Unterschiede zu analysieren.

Methoden: Es wurden 1026 Patienten mit einem schweren Asthma eingeschlossen. Die Erfassung der Patienten-charakteristika erfolgte mit einem Dokumentationsbogen. Es wurden serologisch sIgE gegen 54 Aeroallergene, davon 49 perenniale Allergenen und ein Nahrungsmittelscreen mittels ImmunoCAP $^{\mathrm{TM}}$ ThermoFisher, sowie das Gesamt-IgE und die Eosinophilen im peripheren Blut gemessen

Ergebnisse: Es wiesen 25,2\% der Patienten auch in diesem breiten Allergenspektrum keine Sensibilisierung auf. Bei 74,8\% der Patienten lagen IgE-vermittelte Sensibilisierungen vor: eine bei $8,8 \%$; zwei bei $10 \%$; drei bei $7,7 \%$; $4-10$ bei $26,4 \%$; $11-20$ bei $17,0 \%$ und $\geq 21$ bei $30,1 \%$.

Die häufigsten Sensibilisierungen betrafen: S. aureus-ET, Insekten (Motte, Trogoderma, Schaben), diverse Tierepithelien, Pilze (v. a. Candida a.) sowie Milben (Hausstaub-, Speichermilben), Pollen und Tabakblätter. Der prädiktive Wert anamnestischer Angaben war gering, was ein erweitertes Testspektrum implizieren kann.

Schlussfolgerungen: Ca. drei Viertel $(74,8 \%)$ der Patienten mit einem schweren Asthma wiesen IgE-vermittelte Sensibilisierungen auf, sowohl mono- und oligovalente, als auch ausgesprochen polyvalente Sensibilisierungen. Sensibilisierungen gegen Allergene, die in der klinischen Routine bisher nur eine untergeordnete Rolle spielen sind häufig.

\section{DRUG ALLERGY}

\section{P4.3}

\section{Consistency of response across age, weight and body mass idex subgroups of patients with moderate-to-severe atopic dermatitis on Baricitinib monotherapy}

\section{J.P. Thyssen 1 , A. Torrelo ${ }^{2}$, A. Pink ${ }^{3}$, S. Grond ${ }^{4}$, C. Mert $^{5}$, T. Bieber ${ }^{6}$, U. Boecker ${ }^{7}$}

'Department of Dermatology and Venereology, Bispebjerg Hospital, University of Copenhagen, Copenhagen, Denmark; ${ }^{2}$ Department of Dermatology, Hospital Infantil Univeritario Niño Jesús, Madrid, Spain;

'St John's Institute of Dermatology, Guy's and St Thomas' NHS Foundation Trust, London, UK;

${ }^{4}$ Eli Lilly and Company, Indianapolis, IN, USA;

${ }^{5} \mathrm{HaaPACS} \mathrm{GmbH}$, Schriesheim, Germany;

${ }^{6}$ University of Bonn, Bonn, Germany;

${ }^{7}$ Lilly Deutschland GMBH, Bad Homburg, Germany

Aim: Baricitinib (BARI) (selective Janus kinase[JAK]1/2 inhibitor) is indicated for treatment of moderate-severe atopic dermatitis(AD) in adult candidates for systemic therapy. Aim was to examine effects of age, weight, body mass index(BMI) at baseline on BARI efficacy in BREEZE-AD1, BREEZE-AD2.

Methods: In BREEZE-AD1/AD2 patients with moderate-severe $\mathrm{AD}$ were randomised 2:1:1:1(once-daily placebo, BARI1 mg,2 mg,4 mg[16wk]). Topical, systemic AD treatments were allowed as rescue therapy only; those who received rescue therapy/discontinued treatment were considered nonresponders. This analysis pooled studies to evaluate potential subgroup interaction effects on efficacy based on age $(<25,25-50,>50$ years), weight $(<60,60-<90, \geq 90 \mathrm{~kg})$, BMI $(<25,25-<30, \geq 30 \mathrm{~kg} /$ $\mathrm{m} 2$ ) at baseline in groups tested within the primary endpoint (placebo, BARI2 mg, $4 \mathrm{mg}$ ). Outcomes assessed included proportion of patients achieving vIGA $(0,1)$, EASI75, $\geq 4$ point ItchNRS improvement from baseline. Response rates were reported as relative frequency; treatment-by-subgroup interactions were assessed using a logistic regression model (significance level: $p \leq .10$ ).

Results: 987 patients were stratified by age $(<25$ years [ $n=244] ; 25-50$ years $[n=609]$; $>50$ years $[n=134])$, weight $(<60 \mathrm{~kg}[n=202] ; \quad 60-<90 \mathrm{~kg} \quad[n=641] ; \quad \geq 90 \mathrm{~kg} \quad[n=144])$, $\mathrm{BMI}(<25 \mathrm{~kg} / \mathrm{m} 2[n=549] ; \quad 25-<30 \mathrm{~kg} / \mathrm{m} 2[n=300] ; \quad \geq 30 \mathrm{~kg} /$ $\mathrm{m} 2[n=138])$. Subgroups were well balanced across treatment arms. vIGA $(0,1)$ response rates were consistently higher for BARI2 mg,4 mg than placebo. No significant treatment-by-subgroup interactions were observed for vIGA $(0,1)$ for age, weight, BMI subgroups. Similar results seen for proportion of patients achieving EASI75/Itch NRS $\geq 4$-point improvement, with no significant treatment-by-subgroup interactions observed for either outcome across subgroups.

Conclusion: BARI monotherapy demonstrated consistent beneficial effect in reducing skin inflammation and itch compared to placebo after 16wk of treatment, irrespective of age, weight, BMI at baseline within analysed subgroups. 
P4.4

Erste Daten zur Unverträglichkeit der COVID-19Impfstoffe aus Sicht eines universitären AllergieZentrums

\section{A.-C. Niesert, O. Horvarth, B. Summer, F. Kapp, F. Ruëff, E. M. Oppel}

Klinik und Poliklinik für Dermatologie und Allergologie, LMU München, München, Deutschland

Hintergrund: Zur Eindämmung der COVID-19-Pandemie sind bisher weltweit ca. 2,5 Milliarden Impfdosen verabreicht worden. Erstmals wurde einem Impfstoff der Lipidnanopartikel Polyethylenglykol (PEG) beigefügt. Mögliche Mechanismen einer Unverträglichkeit bei den mRNA-Impfstoffen sind daher gegenüber der mRNA selbst, gegenüber PEG im Sinne einer klassischen Allergie und Reaktionen ausgelöst durch Komplementaktivierung denkbar.

Ziel: Ziel der retrospektiven Datenanalyse ist es, erste Daten zur Diagnostik nach anamnestischer Unverträglichkeit einer COVID-19-Impfung zu präsentieren.

Methode: Es erfolgte eine retrospektive Datenerhebung aller untersuchten Patienten, welche sich mit einer anamnestischen Unverträglichkeit eines COVID-19-Impfstoffes zwischen dem 01.01.2021 und dem 17.06.2021 im Allergie-Zentrum der LMU München vorstellten.

Ergebnisse: Die Datenanalyse umfasste 12 Patienten mit einem Durchschnittsalter von 48,5 Jahren, davon 11 Frauen und ein Mann. In 9 Fällen erfolgte die Verabreichung von Comirnaty (BioNTech/Pfizer), in zwei Fällen die von Vaxzevria $^{\circ}$ (AstraZeneca) und in einem Fall die des COVID-19 Vaccine Moderna ${ }^{\circ}$ (Moderna). Eine Soforttypreaktion wurde von 7 und eine Spättypreaktion von 5 Patienten berichtet. Die Diagnostik bestehend aus Pricktest $(n=8)$, Basophilen-Aktivierungstest $(n=4)$ und Lymphozyten-Transformationstest $(n=2)$ jeweils mit PEG 2000 und Polysorbat 80 ergab keinen Hinweis auf eine Sensibilisierung. Im Fall einer Spätreaktion mit generalisiertem Exanthem nach Comirnaty ${ }^{\circ}$ erfolgte ein Epikutantest mit PEG 2000 und Polysorbat 80, ebenfalls ohne wegweisenden Befund.

Zusammenfassung: Auf Basis der bisher durchgeführten allergologischen Diagnostik konnte in der vorliegenden Patientengruppe keine allergische Reaktion nach Verabreichung eines der drei oben genannten COVID-19-Impfstoffe bestätigt werden. Die bereits in Kasuistiken beschriebenen Exantheme nach Verabreichung des mRNA-Impfstoffes konnten auch bei unseren Patienten beobachtet werden.

\section{P4.6}

\section{PEG-Allergie in Zeiten der Corona-Pandemie - Strategien zur sicheren Vakzinierung}

\section{S. Mathes, K. Brockow, B. Eberlein, T. Biedermann, U. Darsow}

\section{Technische Universität München, München, Deutschland}

Hintergrund: Weltweit erlangt Polyethylenglycol (PEG) als seltenes, verstecktes Allergen Bedeutung. PEG 2000 ist in mRNA-Covid-19-Vakzinen (z. B. Comirnaty", Moderna-Vakzine), das potenzielle Kreuzallergen Polysorbat (PS) 80 in den
Vaxzevria $^{\circ}$ und Janssen ${ }^{\ominus}$-Vakzinen enthalten. Sichere Strategien für Covid-19-Vakzinierungen bei PEG-Allergikern fehlen bisher.

Kasuistiken: Ein 73-jähriger Patient erlitt eine Stunde nach Movicol - Einnahme (enthält PEG 3350) generalisierten Juckreiz und Erytheme. Hauttests zeigten sich positiv für PEG 4000 und 6000; zudem entwickelte der Patient während der Intrakutantestung mit PEG palmare Erytheme und Juckreiz. Da sich im Basophilenaktivierungstest (BAT) positive Reaktionen auf PEG 4000, 6000, Comirnaty - und Moderna ${ }^{\circ}$-Vakzine, negative für PEG 2000, 3350, PS 80 und Vaxzevria ${ }^{\circ}$ zeigten, wurde Vaxzevria ${ }^{\circ}$ stationär fraktioniert verabreicht und vertragen.

Eine 59-jährige Patientin gab an, 5 Minuten nach Lidocainund Triam-Injektion (enthält PEG und PS) vor 5 Jahren mit Urtikaria, Atemnot, Schwindel und Blutdruckabfall, zudem 2019, eine Stunde nach Moviprep Orange-Einnahme, mit Urtikaria, Übelkeit und Erbrechen reagiert zu haben. Prick- und Provokationstestung mit PEG- und PS-freien Triamcinolon-Tabletten, Prilocain und Ultracain D-S ${ }^{\circledR}$ zeigten keine Reaktionen. In einem Pricktest zeigten sich Sensibilisierungen gegen PEG 4000 und 6000, zudem berichtete die Patientin über starke Übelkeit während der Testung. Ein BAT erbrachte negative Ergebnisse für PEG 2000, 3350, 4000, 6000, PS 80, Vaxzevria ${ }^{\circ}$ und Janssen ${ }^{\circledR}$ bei grenzwertigen Ergebnissen für Comirnaty und das Moderna ${ }^{\oplus}$ Vakzin. Der Patientin wurde eine stationäre, fraktionierte Impfung mit Vaxzevria oder Janssen ${ }^{\bullet}$ empfohlen.

Kommentar: Durch stufenweise Hauttests und Ergänzung mittels zellbasierter Diagnostik konnten die Sensibilisierungsprofile von zwei PEG-allergischen Patienten herausgearbeitet und eine sichere Covid-19-Vakzinierung ermöglicht werden.

\section{P4.7}

Arzneimittelexanthem mit multiplen Antibiotika als Auslöser - Herausforderungen allergologischer Diagnostik

\section{S. Traidl, D. Wieczorek, A. Kapp, B. Wedi}

Klinik für Dermatologie, Allergologie und Venerologie, Comprehensive Allergy Center, Hannover, Deutschland

Unerwünschte Arzneimittelwirkungen betreffen etwa 15-25\% der Patienten und manifestieren sich regelhaft an der Haut. Eine zunehmende Polypharmazie, andere Ursachen der Effloreszenzen und unklare Anamnesen erschweren die Identifikation des auslösenden Agens. Daher ist eine umfassende diagnostische Abklärung erforderlich, um eine ArzneimittelÜberempfindlichkeit zu diagnostizieren. Wir berichten über die komplexe, allergologische Diagnostik bei zwei Fällen von schweren makulopapulösen Exanthemen durch die Einnahme von verschiedenen Antibiotika. Ein 8-jähriger Junge mit Pneumonie bei Mukoviszidose erhielt zwei Wochen lang Meropenem, Vancomycin und Fosfomycin. Vier Tage später entwickelte er ein Exanthem. Serum-IgE sowie Prick- und Intrakutan-Testung zeigten sich unauffällig. Meropenem, Vancomycin und Amoxicillin ergaben jedoch positive Ergebnisse im Lymphozytentransformationstest und Epikutantest. Ein 52-jähriger Patient erhielt aufgrund einer bakteriellen Endokarditis multiple Medikamente. Im Verlauf entwickelte er eine Erythrodermie. Als mögliche Auslöser kamen Doxycyclin, Rifampicin, Vancomycin, Meropenem, Ciprofloxacin und Diclofenac in Frage. Amoxicillin und Ampicillin zeigten positive Ergebnisse im Prick-Test. Der Intrakutantest auf Meropenem erbrachte eine ausgeprägte bullöse Reaktion. Der Epikutantest zeigte Spättypreaktionen gegen Penicillin und Aminopenicilline sowie 
Vancomycin und Meropenem. Provokationen mit Doxycyclin und Rifampicin führten zu Reaktionen. Die beiden Fälle unterstreichen die Bedeutung sowie die Komplexität allergologischer Abklärungen bei Antibiotika-Überempfindlichkeit. Spezifische IgE-Tests sind nur für wenige Antibiotika aktuell verfügbar. Die Anwendung der Medikamente im Pricktest sowie im Intrakutan- und Epikutantest ist zumeist spezialisierten Zentren vorbehalten. Es ist jedoch gerade im Hinblick auf eine Zunahme von Antibiotikaresistenzen für die Patienten wichtig, mögliche Auslöser zu identifizieren.

\section{P4.8}

Anaphylaxie auf multiple Medikamente und deren Bedeutung für Impfstoffapplikationen

\section{J. Pickert', 2, I. Hennighausen', S. Mühlenbein', C. Möbs ${ }^{2}$, W. Pfützner ${ }^{1,2}$}

${ }^{1}$ Allergiezentrum Hessen, Universitätsklinikum Marburg, Marburg, Deutschland;

${ }^{2}$ Klinisch-Experimentelle Allergologie, PhilippsUniversitätMarburg, Marburg, Deutschland

Soforttypallergien gegenüber Arzneimittelzusatzstoffen wie Polyethylenglycol (PEG) bzw. Macrogol sind insbesondere in diesem Jahr durch die Anwendung PEG-haltiger Covid-19 mRNA Impfstoffe und möglichen anaphylaktischen Reaktionen hierauf in den Fokus gerückt. Eine 24-jährige Patientin stellte sich zur Abklärung wiederholter Anaphylaxien jeweils 5-10 Minuten nach Administration unterschiedlicher Arzneimittel vor. Hierbei handelte es sich um Tabletten mit den Inhaltsstoffen Sultamicillin bzw. Metamizol und einem DiclofenacSchmerzgel. Ibuprofen wurde regelmäßig vertragen. In der allergologischen Diagnostik zeigten sich Gesamt-IgE, spezifisches IgE gegen Betalaktame und Tryptase unauffällig. Pricktests erbrachten positive Ergebnisse für Arzneimittelpräparate mit den Inhaltsstoffen Penizillin V, Metamizol und Ibuprofen, nicht jedoch für Diclofenac- und Sultamicillin-haltige Medikamente. Im Verlauf wurde von einer weiteren Anaphylaxie nach Ablecken eines Löffel Joghurts vermengt mit Kinderlax (Macrogol 4000 ) berichtet. Dieses zeigte sich im anschließenden Pricktest ebenfalls positiv. In Zusammenschau aller Testbefunde ergab sich, dass ausschließlich die Medikamente, die PEG eines Molekulargewichts $\geq 4000$ enthielten positive Pricktestreaktionen hervorriefen. Anschließende orale Provokationstests mit PEG-freien Arzneimittelpräparaten mit den Inhaltsstoffen Penizillin V, Metamizol bzw. Ibuprofen wurden toleriert. Ein sodann durchgeführter Intrakutantest mit dem Covid-19 Impfstoff Comirnaty (1:100 verdünnt) führte zur akuten Dyspnoe bei einer $11 \mathrm{~mm}$ großen Quaddel im Hauttest. Dieser Fall zeigt, dass bei multiplen allergischen Reaktionen auf unterschiedliche Medikamente eine Allergie gegen deren Zusatzstoffe in Betracht gezogen werden muss. Hierzu gehört auch PEG, das u.a. als Stabilisator und aufgrund seiner hygroskopischen Eigenschaften in Arzneimitteln zu finden ist. Zu beachten ist, dass Hauttests mit PEG(-haltigen Substanzen) das Risiko einer Anaphylaxie bergen.

\section{HEREDITARY ANGIOEDEMA}

\section{P4.10}

Eine randomisierte, doppelblinde, placebokontrollierte monozentrische PhaseI-Studie mit ansteigender Einzeldosis zur Untersuchung der Sicherheit, Verträglichkeit und Pharmakokinetik von intravenös (i. v.) und subkutan (s. c.) verabreichtem Garadacimab (CSL312) bei gesunden Freiwilligen

\section{A. McKenzie ${ }^{1}$, A. Roberts ${ }^{1}$, S. Malandkar', H. Feuersenger ${ }^{2}$, C. Panousis ${ }^{1}$, D. Pawaskar ${ }^{3}$}

${ }^{1}$ CSL Limited, Parkville, Victoria, Australien;

${ }^{2} \mathrm{CSL}$ Behring Innovation GmbH, Marburg, Deutschland; ${ }^{3} \mathrm{CSL}$ Behring, King of Prussia, PA, USA

Hintergrund: Garadacimab ist ein vollständig humaner monoklonaler G4-Immunglobulin-Antikörper der den aktivierten Faktor XII (FXIIa) hemmt. In einer Phase-I-Studie wurde die Sicherheit, Verträglichkeit und das pharmakokinetische (PK)/ pharmakodynamische (PD) Profil nach einmaliger Gabe von Garadacimab bei gesunden Probanden untersucht.

Methoden: Die Studienteilnehmer wurden in fünf i.v. (0,1; 0,$3 ; 1 ; 3$ oder $10 \mathrm{mg} / \mathrm{kg}$ ) und drei s. c. Studienarmen (1, 3 oder $10 \mathrm{mg} / \mathrm{kg}$ ) randomisiert. Garadacimab oder Placebo wurde im Verhältnis 2:1 an jeweils sechs Probanden pro Studienarm gegeben. Der Beobachtungszeitraum zur Sicherheit umfasste 85 Tage nach der Dosierung. Blutproben für die PK/PD wurden über die gesamte Studiendauer analysiert.

Ergebnisse: Es wurden keine Todesfälle, schwerwiegende Nebenwirkungen (TEAE) oder TEAE, die zum Studienabbruch führten, in 48 männlichen Probanden (Mittelwert, Standardabweichung: 27,4; 6,4 Jahre) berichtet. Häufigkeit und Schwere der TEAE zeigten keine Dosisabhängigkeit. Es wurden keine Antikörper gegen Garadacimab nachgewiesen. Die mittlere maximale Plasma-Konzentration war dosisabhängig (i.v. und s. c.). Die maximale Plasmakonzentration (Tmax) war nach 1,0 Stunde (Infusionsende; 3,5 Stunden bei $0,1 \mathrm{mg} / \mathrm{kg}$ ) in den i.v. Armen, nach 5,6-7,0 Tagen in den s. c. Armen erreicht. Die mittlere Halbwertszeit bei den i.v. Armen betrug 14,3-20,4 Tage, bei den s.c. Armen 18,2-19,6 Tage. Die Bioverfügbarkeit nach der s. c. Gabe betrug 49,7\%. Garadacimab (i.v. und s. c.) zeigte eine dosisabhängige Hemmung der Kallikrein-Aktivität mit keiner oder minimaler Restaktivität bei höheren Dosen.

Schlussfolgerungen: Die einmalige i.v. oder s.c. Gabe von Garadacimab an Probanden war sicher und gut verträglich. Es wurden ein dosisabhängiger Anstieg der Plasma-Konzentration, gefolgt von einer Hemmung des Kallikrein-Kinin-Signalwegs, festgestellt. Ergebnisse dieser Studie dienten der Planung weiterer klinischer Studien, einschließlich einer Phase-II-Studie in HAE-Patienten. 
Use of subcutaneous C1 inhibitor in pregnancy, lactation and beyond - Long-term follow up of patient and her child

\section{S. Andarawewa, E. Aygören-Pürsün}

Department of Children and Adolescents, University Hospital Frankfurt, Goethe University, Frankfurt, Germany

Background: Hereditary angioedema (HAE) is a rare and potentially life-threatening disorder characterised by attacks of angioedema due to deficient or dysfunctional $\mathrm{Cl}$ inhibitor (C1-INH). Replacement of C1-INH with a subcutaneous (SC) plasma-derived concentrate is an approved therapy for patients with HAE. We report the long-term follow up of a patient with HAE and her child, where the patient was treated with C1-INH (SC) throughout pregnancy and lactation.

Results: A 40-year-old female was diagnosed with HAE Type I at the age of 17 years after experiencing her first attack. The patient had 0-2 attacks per year, which were treated with 500-1000 IU intravenous (IV) C1-INH. The patient's disease worsened in pregnancy. At the end of the second trimester she was experiencing 2 attacks/week, which included a laryngeal attack. Due to reduced quality of life and poor venous access, the patient was prescribed $15 \mathrm{IU} / \mathrm{kg}$ Cl-INH (SC) twice a week. Under this regimen, she was attack-free until delivery. The patient had a spontaneous vaginal delivery, in the 38th gestational week, of a healthy baby girl. The baby had an Apgar score of 10/10, a birth weight of $3.505 \mathrm{~kg}$, a length of $50 \mathrm{~cm}$ and a head circumference of $34.5 \mathrm{~cm}$. The baby was breastfed for 1 year. Post-partum, the patient experienced attacks when she attempted to reduce her dose to once a week, so she resumed her previous regimen. The patient was then attack-free until 10 months after delivery, when she had uvula swelling and uncertain laryngeal oedema symptoms 3-4 times over the following 2-3 weeks. Due to the location of the attacks, the dose was increased to $60 \mathrm{IU} / \mathrm{kg} \mathrm{Cl}$-INH (SC) twice a week. No health impairment was reported for more than 24 months of follow up in this patient or her child.

Conclusions: C1-INH (SC) provided effective prophylaxis for this patient with HAE during pregnancy and lactation. Even low-dose C1-INH (SC) prophylaxis can be effective during pregnancy and lactation in some patients with HAE.
Ergebnisse einer randomisierten, doppelblinden, placebokontrollierten Phase-II-Studie zur Untersuchung der Sicherheit und Wirksamkeit von Garadacimab (CSL312), einem Faktor XIla inhibierenden monoklonalen Antikörper zur Prophylaxe des hereditären Angioödems

T. Craig1, M. Magerl', D. S Levy ${ }^{3}$, A. Reshef ${ }^{4}$, W. R Lumry ${ }^{\text {5 }}$, I. Martinez-Saguer ${ }^{6}$, J. S Jacobs ${ }^{7}$, W. H Yang ${ }^{8}$, B. Ritchie ${ }^{9}$, E. Aygören-Pürsün ${ }^{10}$, P. K Keith 11 , P. Busse ${ }^{12}$, H. Feuersenger ${ }^{13}$, I. Jacobs ${ }^{14}$, I. Pragst ${ }^{13}$

${ }^{1}$ Allergy, Asthma and Immunology, Departments of Medicine and Pediatrics, Penn State University, Hershey, PA, USA; ${ }^{2}$ Dermatological Allergology, Allergie-Centrum-Charité, Department of Dermatology and Allergy, Charité Universitätsmedizin Berlin, Berlin, Germany;

${ }^{3}$ Division of Allergy and Immunology, University of California, Irvine, CA, USA;

${ }^{4}$ Allergy, Immunology and Angioedema Center, Barzilai University Hospital, Ashkelon, Israel;

${ }^{5}$ AARA Research Center, Dallas, TX, USA;

${ }^{6}$ HZRM Haemophilia Center Rhein Main, Mörfelden-Walldorf, Germany;

${ }^{7}$ Allergy and Asthma Clinical Research, Walnut Creek, CA, USA;

${ }^{8}$ Ottawa Allergy Research Corporation, Department of Medicine, University of Ottawa, Ottawa, ON, Canada; ${ }^{9}$ Division of Hematology, Department of Medicine, University of Alberta, Edmonton, AB, Canada;

${ }^{10} \mathrm{Klinik}$ für Kinder- und Jugendmedizin;

Johann Wolfgang-Goethe Universität, Frankfurt, Germany;

${ }^{11}$ McMaster University Medical Center, Hamilton, ON,

Canada;

${ }^{12}$ Division of Clinical Immunology and Allergy, Department of Medicine, Icahn School of Medicine at Mount Sinai, New York, NY, USA;

${ }^{13} \mathrm{CSL}$ Behring Innovation GmbH, Marburg, Germany; ${ }^{14} \mathrm{CSL}$ Behring, King of Prussia, PA, USA

Hintergrund: Der aktivierte Faktor XII (FXIIa) ist im Rahmen der Kontaktaktivierung entscheidend für die Freisetzung von Bradykinin, dem Hauptmediator des hereditären Angioödems (HAE). Garadacimab (CSL312), ist ein vollständig humaner monoklonaler Immunglobulin-G4-Antikörper, der FXIIa hemmt. Ziel dieser Phase-II-Studie (CSL312_2001; NCT03712228) war die Untersuchung der Pharmakokinetik, Sicherheit und Wirksamkeit des subkutan (s.c.) verabreichten Garadacimab zur Prophylaxe von Attacken in HAE.

Methoden: Randomisierte HAE Typ I/II Patienten erhielten über 12 Wochen alle 4 Wochen entweder Placebo oder s.c. Garadacimab 75, 200 oder $600 \mathrm{mg}$. Eine Woche vor der ersten s.c. Dosis wurde einmalig eine intravenöse Initialdosis von jeweils 0, 40, 100 bzw. $300 \mathrm{mg}$ Garadacimab verabreicht. Der primäre Endpunkt war die monatliche Rate von HAE Attacken. Weitere Endpunkte umfassten die Reduktion von Attacken im Vergleich zur Run-in Periode oder zu Placebo sowie die Untersuchung von Nebenwirkungen.

Ergebnisse: Es wurden insgesamt 32 erwachsene Patienten mit HAE Typ I (93,75\%) und Typ II $(6,25 \%)$ randomisiert. Von diesen waren 90,63\% Kaukasier mit einem Anteil von 56,25\% Frauen. In der Run-in Periode war die durchschnittliche monat- 
Tab. 1 Wichtigste Daten zur Wirksamkeit und Sicherheit

\begin{tabular}{|c|c|c|c|c|}
\hline \multirow[b]{2}{*}{ Parameter } & \multicolumn{4}{|l|}{$N=32$} \\
\hline & $\begin{array}{l}\text { Placebo } \\
q 4 \mathrm{w} \\
(n=8)\end{array}$ & $\begin{array}{l}75 \mathrm{mg} \text { Garadacimab } \mathrm{q} 4 \mathrm{w} \\
(n=9)\end{array}$ & $\begin{array}{l}200 \mathrm{mg} \text { Garadacimab } \\
\mathrm{q} 4 \mathrm{w} \\
(n=8)\end{array}$ & $\begin{array}{l}600 \mathrm{mg} \text { Garadacimab } \\
\mathrm{q} 4 \mathrm{w} \\
(n=7)\end{array}$ \\
\hline $\begin{array}{l}\text { Mittelwert HAE Attackenraten pro Monat wäh- } \\
\text { rend der Run-in Period, (Median) }\end{array}$ & $\begin{array}{l}5,1 \\
(4,6)\end{array}$ & $\begin{array}{l}6,1 \\
(6,3)\end{array}$ & $\begin{array}{l}5,7 \\
(5,7)\end{array}$ & $\begin{array}{l}3,5 \\
(3,0)\end{array}$ \\
\hline $\begin{array}{l}\text { Mittelwert HAE Attackenraten pro Monat wäh- } \\
\text { rend der Behandlungsperiode, (Median) }\end{array}$ & $\begin{array}{l}4,2 \\
(4,6)\end{array}$ & $\begin{array}{l}0,5 \\
(0,0)\end{array}$ & $\begin{array}{l}0,1 \\
(0,0)\end{array}$ & $\begin{array}{l}0,4 \\
(0,3)\end{array}$ \\
\hline $\begin{array}{l}\text { Patienten mit HAE Attackenreduktion ( } \geq 90 \% \text { ) } \\
\text { vs. Run-In Periode bei Respondern, } \%\end{array}$ & 0,0 & 88,9 & 100,0 & 57,1 \\
\hline Patienten ohne Attacken, \% & 0,0 & 55,6 & 87,5 & 42,9 \\
\hline $\begin{array}{l}\text { Mittelwert behandelte HAE Attacken pro Monat } \\
\text { (Median) }\end{array}$ & $\begin{array}{l}4,0 \\
(4,4)\end{array}$ & $\begin{array}{l}0,4 \\
(0,0)\end{array}$ & $\begin{array}{l}0,1 \\
(0,0)\end{array}$ & $\begin{array}{l}0,2 \\
(0,0)\end{array}$ \\
\hline TEAE, $n(\%)$ & $3(37,5)$ & $2(22,2)$ & $1(12,5)$ & $5(71,4)$ \\
\hline Reaktion an der Injektionsstelle, $n(\%)$ & $2(25,0)$ & $1(11,1)$ & $1(12,5)$ & $4(57,1)$ \\
\hline
\end{tabular}

lichen HAE Attackenrate 5,17. Während der Behandlung mit Placebo, 75, 200 und 600 mg Garadacimab betrugen die Mittelwerte der monatlichen Attackenrate jeweils 4,24; 0,48; 0,05 und 0,40 (Tab. 1). Der Prozentsatz der Patienten mit einer oder mehr Nebenwirkung unter Garadacimab war vergleichbar zu Placebo. Alle Nebenwirkungen waren mild oder moderat, schwere Nebenwirkungen traten nicht auf. Die häufigste Nebenwirkung waren milde oder moderate Erytheme an der Injektionsstelle $(12,5 \%)$. Alle Patienten beendeten die Studie erfolgreich.

Schlussfolgerungen: Die monatliche s. c. Gabe von Garadacimab wurde gut vertragen und war bei der Prävention von Attacken wirksam. Diese Studie liefert die erste klinische Evidenz für die Relevanz von FXIIa in HAE.

\section{P5.6}

\section{Reduction in attacks in hereditary angioedema} (HAE) with berotralstat is consistent regardless of prior prophylactic treatment: A subgroup analysis of the phase $3 \mathrm{APeX}-2$ trial

\section{J. Anderson ${ }^{1}$, R. Gagnon ${ }^{2}$, B. Desai ${ }^{3}$, D. Tomita ${ }^{3}$, K. Aggarwal ${ }^{3}$, K.V. Sitz ${ }^{4}$}

${ }^{1}$ Alabama Allergy \& Asthma Center, Homewood, AL, USA; ${ }^{2}$ Clinique Spécialisée en Allergie de la Capitale, Québec, QC, Canada;

${ }^{3}$ BioCryst Pharmaceuticals, Inc, Durham, NC; USA; ${ }^{4}$ Little Rock Allergy \& Asthma Clinic P.A, Little Rock, AR, USA

Rationale: Prophylactic treatment in the management of HAE is common. Berotralstat is an oral once-daily selective plasma kallikrein inhibitor that was shown to reduce HAE attack rates in a Phase 3 study (NCT03485911). This post hoc analysis evaluated the efficacy of berotralstat in patients previously treated with prophylactic medications.

Methods: A total of 121 patients were randomized to berotralstat $110 \mathrm{mg}: 150 \mathrm{mg}$ : placebo daily for 24 weeks. Investigatorconfirmed attacks were analyzed for patients grouped by type of prior prophylaxis: prior $\mathrm{Cl}$ esterase inhibitor (C1-INH), prior androgen, and no prior prophylaxis. Prior C1-INH and prior androgen categories were not mutually exclusive.
Results: Overall, $75 \%$ of patients in the berotralstat $150 \mathrm{mg}$ dose group and $73 \%$ in the placebo group had prior prophylactic treatment. Among patients with prior C1-INH prophylaxis or prior androgen use, berotralstat $150 \mathrm{mg}$ significantly reduced attacks compared to placebo during the treatment period (C1-INH, 1.58 attacks/month vs placebo 2.84 attacks/month, $p=0.012$; androgens, 1.35 attacks/month vs placebo 2.60 attacks/month, $p<0.001)$. Lastly, patients without prior prophylaxis had a reduction in attacks ( 0.86 attacks/month compared with 1.78 attacks/month in placebo; $p=0.056$ ).

Conclusion: In this subgroup analysis, patients with prior C1-INH prophylaxis or prior androgen use treated with berotralstat demonstrated a significant reduction in attacks vs. placebo, making oral berotralstat a valuable potential preventive treatment option for patients with HAE.

Encore-submission, original abstract has been published by the AAAAI in the 2021 Meeting Abstracts Supplement in JACI

\section{P5.7}

\section{Berotralstat reduces use of on-demand} medication in hereditary angioedema (HAE) patients previously treated with prophylactic therapies

\section{R. Gower ${ }^{1}$, P. Busse ${ }^{2}$, B. Desai ${ }^{3}$, D. Tomita ${ }^{3}$, K. Aggarwal ${ }^{3}$, Ta. Kinaciyan ${ }^{4}$}

${ }^{1}$ Marycliff Clinical Research, Spokane, WA, USA; 2Division of Allergy and Immunology, Department of Medicine, Icahn School of Medicine at Mount Sinai, New York, NY; USA;

${ }^{3}$ BioCryst Pharmaceuticals, Inc, Durham, NC, USA; ${ }^{4}$ Department of Dermatology, Medical University of Vienna, Vienna, Austria

Rationale: The goal of HAE prophylaxis is to minimize the number of HAE attacks and the associated disease burden, including treatment with on-demand medications. Berotralstat is an oral, once-daily selective plasma kallikrein inhibitor that has been shown to reduce attack frequency in a Phase 3 study (NCT03485911). This analysis evaluates reduction in ondemand medication in patients with prior prophylaxis. 
Methods: A double-blind, placebo-controlled study (APeX2) randomized patients to berotralstat $110 \mathrm{mg}(n=41): 150 \mathrm{mg}$ $(n=40)$ : placebo $(n=40)$ daily for 24 weeks in Part 1. For this post hoc analysis, patients were grouped according to their prior prophylaxis: prior $\mathrm{Cl}$ esterase inhibitor (C1-INH), prior androgen, or no prior prophylactic medication. Prior C1-INH and prior androgen categories are not mutually exclusive.

Results: In patients with prior C1-INH prophylaxis (berotralstat $150 \mathrm{mg} n=21$; placebo $n=16$ ), the rate of use of ondemand treatment was significantly reduced vs placebo $(-59.2 \%, p=0.002)$. Similar reductions were noted for patients with prior androgen use (berotralstat $150 \mathrm{mg} n=22$; placebo $n=25)(51-.8 \% ; p=0.004)$ and in patients without prior prophylaxis (berotralstat $150 \mathrm{mg} n=10$; placebo $n=10)(71-.2 \%$; $p=0.019$ ). The rate reduction corresponds to approximately 2.2 fewer doses of on-demand medication per month compared to placebo in patients with prior C1-INH use, 1.6 for those with prior androgen use, and 1.5 for those with no prior prophylaxis.

Conclusion: Prophylactic treatment with oral berotralstat $150 \mathrm{mg}$ resulted in significant reductions in on demand-medication compared to placebo irrespective of prior prophylactic treatment.

Encore-submission, original abstract has been published by the AAAAI in the 2021 Meeting Abstracts Supplement in JACI

\section{P5.8}

Berotralstat consistently demonstrates reductions in attack frequency in hereditary angioedema (HAE) irrespective of baseline attack Rate: Subgroup analysis from the APeX-2 trial

\section{H. H. Li', B. Desai' ${ }^{2}$ D. Tomita², R. Tachdijan ${ }^{3}$}

${ }^{1}$ Institute for Asthma and Allergy, Chevy Chase, MD, USA; ${ }^{2}$ BioCryst Pharmaceuticals, Inc, Durham, NC, USA; ${ }^{3}$ AIRE Medical of Los Angeles, Santa Monica, CA, USA

Rationale: Berotralstat is an oral plasma kallikrein inhibitor in development for HAE attack prophylaxis. HAE is characterized by unpredictable, episodic attacks; some patients experience frequent attacks without treatment. This analysis sought to understand whether baseline attack frequency correlates with responder rates with berotralstat.

Methods: 121 patients were randomized to berotralstat $110 \mathrm{mg}$ : $150 \mathrm{mg}$ : placebo daily for 24 weeks in a phase 3 double-blind, placebo-controlled study (NCT03485911). This post hoc analysis examined the reduction of HAE attacks by baseline attack rate Cohort 1: $<2$ attacks/month; Cohort 2: $\geq 2$ to $<4$ attacks/month; Cohort 3: $\geq 4$ attacks/month.

Results: In Cohort 1, median baseline attack rates per month were 1.3 (berotralstat $150 \mathrm{mg} ; n=10$ ) and 1.7 (placebo; $n=12$ ) which declined to 0.41 and 1.3 , respectively. In Cohort 2, median baseline attack rates per month were 2.7 (berotralstat $150 \mathrm{mg} ; n=20$ ) and 3.1 (placebo; $n=21$ ) which declined to 1.2 and 2.7, respectively. For Cohort 3 , median baseline attack rates per month were 5.2 with berotralstat $150 \mathrm{mg}(n=10)$ and 4.5 with placebo $(n=6)$ and declined to 1.9 and 2.5 , respectively. In Cohorts 1, 2, and 3, treatment with berotralstat $150 \mathrm{mg}$ resulted in $\mathrm{a} \geq 50 \%$ relative reduction in attack rate in $70 \%, 55 \%$, and $50 \%$ of patients, respectively. In addition, $60 \%, 45 \%$, and $50 \%$ of patients, respectively, had $\geq 70 \%$ relative reduction in attack rate.

Conclusion: These results demonstrate consistent responder rates with berotralstat, adding a potential oral pro- phylactic option to the treatment armamentarium for physicians.

Encore-submission, original abstract has been published by the AAAAI in the 2021 Meeting Abstracts Supplement in JACI

\section{P5.9}

Was sagen die Patienten? Ergebnisse einer Online-Umfrage unter HAE-Patienten in Deutschland

\section{Magerl' ${ }^{1}$, I. Martinez-Saguer ${ }^{2}$, L. Schauf ${ }^{3}$, S. Pohl ${ }^{4}$, K. Brendel ${ }^{5}$}

${ }^{1}$ Klinik für Dermatologie, Venerologie und Allergologie, Charité-Universitätsmedizin Berlin, Berlin, Deutschland; ${ }^{2}$ Hämophilie-Zentrum Rhein-Main, Mörfelden-Walldorf, Deutschland;

${ }^{3} \mathrm{HAE}-$ Vereinigung e. V., Aldenhoven/Siersdorf, Deutschland; ${ }^{4}$ BioCryst Pharma GmbH, München, Deutschland;

${ }^{5}$ Primus Consulting Group $\mathrm{GmbH}$, Martinsried, Deutschland

Einführung: Das Hereditäre Angioödem (HAE) ist eine seltene Erbkrankheit, die Patienten und Angehörige belastet. Die hier vorgestellten Ergebnisse einer 2021 durchgeführten Umfrage unter HAE Patienten dokumentieren die Lebensumstände im Verlauf und die Wünsche der Patienten.

Methoden: In Zusammenarbeit mit der Selbsthilfegruppe HAE-Vereinigung e.V. wurde ein Online-Fragebogen mit 77 Fragen entwickelt, um Daten zur Anamnese, Lebenssituation, Diagnose und Behandlung von HAE-Patienten zu erfassen. Im Zeitraum Jan-Mai 2021 nahmen insgesamt 86 Patienten an der Befragung teil.

Ergebnisse: Vor der Stellung der Diagnose HAE gaben $55 \%$ eine starke oder sehr starke Beeinträchtigung im täglichen Leben an $(N=67)$, die sich nach Erhalt der gesicherten Diagnose HAE bei $67 \%$ der Befragten stark oder sehr stark verringerte $(N=63)$. Bis zur Diagnosestellung vergingen im Median 8 Jahre $(1 \mathrm{~m}-50 \mathrm{a})$, sie erfolgte bei älteren Patienten deutlich später als bei jüngeren. Für die vergangenen 3 Monate wurden im Median 2 Attacken (0-58) mit einer medianen Dauer von $24 \mathrm{~h}(1-180 \mathrm{~h})$ angegeben $(N=84), 29$ Patienten waren ohne Attacke in diesem Zeitraum. Patienten unter Langzeitprophylaxe (LZP) berichteten eine geringere Häufigkeit an Attacken und erreichten häufiger eine gute Krankheitskontrolle (AECTScores $\geq 10$ Punkte $57 \%$; $=30$ ohne, $77 \%$; $N=47$ mit LZP). Sie nahmen u. a. Durchbruchsattacken (35\%), eine geringere Compliance (23\%) sowie einen höheren Aufwand (19\%) als Schwächen der aktuell verfügbaren LZP wahr $(N=48)$. Diese Daten mögen teilweise erklären, weshalb ein großes Interesse an einer Tablette besonders unter LZP-Patienten $(82 \% ; N=48)$ besteht.

Zusammenfassung: Die Zeit bis zur Diagnosestellung bei HAE Patienten ist weiterhin zu hoch und die große Mehrzahl leidet weiterhin unter den Attacken. Patienten unter LZP haben weniger Attacken, eine bessere Krankheitskontrolle und Lebensqualität. Das Interesse an einer weiteren Verbesserung durch eine orale Prophylaxe ist unter den Befragten sehr groß. 


\section{ATOPIC DERMATITIS}

\section{P5.1}

\section{Dupilumab in adolescents with moderate-to- severe atopic dermatitis and a history of allergic rhinitis: Subgroup analysis from a phase 3 trial}

\section{Sher ${ }^{1}$, T. Buhl ${ }^{2}$, W. Soong ${ }^{3}$, M. Bastian ${ }^{4}$, Z. Chen ${ }^{5}$,} A. Bansal ${ }^{5}$

${ }^{1}$ Peninsula Research Associates, Rolling Hills Estates, CA, USA;

${ }^{2}$ University Medical Center Göttingen, Göttingen, Germany; ${ }^{3}$ Alabama Allergy \& Asthma Center, Birmingham, AL, USA ${ }^{4}$ Sanofi, Frankfurt, Germany;

${ }^{5}$ Regeneron Pharmaceuticals, Inc., Tarrytown, NY, USA

Background: Atopic dermatitis (AD) is a chronic inflammatory skin disease commonly associated with other atopic diseases such as allergic rhinitis (AR). Dupilumab is approved in the EU for adults and adolescents with moderate-to-severe $\mathrm{AD}$ and for children with severe $\mathrm{AD}$; as an add-on maintenance treatment for adults and adolescents for severe asthma with type 2 inflammation; and as an add-on treatment with intranasal corticosteroids for the treatment of adults with severe chronic rhinosinusitis with nasal polyps. This subgroup analysis aimed to determine whether a history of AR impacts the efficacy of dupilumab in adolescents with moderate-to-severe AD.

Methods: In this double-blind, placebo-controlled, phase 3 trial (NCT03054428), patients (age 12-17 years) were randomized 1:1:1 to dupilumab $300 \mathrm{mg}$ every 4 weeks (q4w; $n=84$ ); dupilumab $200 \mathrm{mg}$ or $300 \mathrm{mg}$ q2w $(n=82)$; or placebo q2w $(n=85)$, for 16 weeks.

Results: 50/59/57 patients reported history of AR at baseline in the $\mathrm{q} 4 \mathrm{w} / \mathrm{q} 2 \mathrm{w} /$ placebo groups, respectively. At Week 16, regardless of history of AR, more patients receiving dupilumab $\mathrm{q} 4 \mathrm{w} / \mathrm{q} 2 \mathrm{w}$ vs placebo achieved Investigator's Global Assessment score 0/1 (with AR: $22.0 \% / 25.4 \%$ vs $3.5 \%$; without AR: $11.8 \% / 21.7 \%$ vs $0 \%$ ) and $\geq 75 \%$ improvement in Eczema Area and Severity Index (with AR: $36.0 \% / 44.1 \%$ vs $8.8 \%$; without AR: $41.2 \% / 34.8 \%$ vs $7.1 \%)$. Similar results were observed for $\geq 4$-point improvement from baseline in pruritus Numerical Rating Scale score (with AR: $26.5 \% / 37.3 \%$ vs $5.4 \%$; without AR: $26.5 \% / 34.8 \%$ vs $3.6 \%$ ) and $\geq 6$-point improvement from baseline in Children's Dermatology Life Quality Index (with AR: $62.8 \% / 60.0 \%$ vs $22.0 \%$; without AR: $53.6 \% / 61.9 \%$ vs $15.4 \%$ ).

Conclusion: Dupilumab improved AD signs and symptoms in adolescents with moderate-to-severe $\mathrm{AD}$ regardless of history of AR.

\section{P5.2}

Dupilumab improves signs and symptoms of severe atopic dermatitis in children aged 6-11 years with and without comorbid allergic rhinitis

\section{A. Beck ${ }^{1}$ A. Wollenberg ${ }^{2}$, E. Siegfried ${ }^{3}$, Z. Chen ${ }^{4}$, M. Bastian ${ }^{5}$, A. Abramova ${ }^{4}$}

1University of Rochester Medical Center, Rochester, NY, USA;

${ }^{2}$ Ludwig-Maximilian University, Munich, Germany;

'Saint Louis University and Cardinal Glennon Children's Hospital, St. Louis, MO, USA;

${ }^{4}$ Regeneron Pharmaceuticals, Inc., Tarrytown, NY, USA; ${ }^{5}$ Sanofi, Frankfurt, Germany

Background: Atopic dermatitis (AD) is a chronic inflammatory skin disease that frequently occurs with other type 2 inflammatory diseases, including allergic rhinitis (AR). Dupilumab inhibits signalling of interleukin (IL)-4 and IL-13-key drivers of type 2-mediated inflammation in multiple diseases, including AD and AR. This subgroup analysis evaluated the efficacy of dupilumab with concomitant topical corticosteroids (TCS) for severe $\mathrm{AD}$ in children with and without comorbid $\mathrm{AR}$.

Methods: In this double-blind, 16 -week, phase 3 trial (NCT03345914), 367 children aged 6-11 years were randomized 1:1:1 to $300 \mathrm{mg}$ dupilumab every 4 weeks (q4w), weight-based $100 / 200 \mathrm{mg}$ dupilumab every 2 weeks (q2w), or placebo, with concomitant medium-potency TCS. AR history was ascertained by caregiver report.

Results: At baseline, $61 \% / 61 \% / 63 \%$ patients in the $\mathrm{q} 4 \mathrm{w} /$ q2w/placebo groups reported a history of AR; $39 \% / 39 \% / 37 \%$ patients had no AR history. Baseline disease severity was comparable in both subgroups. At Week 16, more patients receiving dupilumab $\mathrm{q} 4 \mathrm{w} / \mathrm{q} 2 \mathrm{w}$ vs placebo achieved Investigator's Global Assessment (IGA) score 0/1 (with AR:32.0\%/30.7\% vs 9.1\%; without AR:34.0\%/27.7\% vs $15.2 \%), \geq 75 \%$ improvement in Eczema Area and Severity Index (with AR:68.0 \%/69.3\% vs $20.8 \%$; without AR: $72.3 \% / 63.8 \%$ vs $37.0 \%$ ), and $\geq 4$-point reduction from baseline in Peak Pruritus Numerical Rating Scale (with AR:50.0\%/56.2\% vs $13.2 \%$; without AR:52.2\%/61.7\% vs $10.9 \%) ; P<0.05$ between verum groups and placebo except $\mathrm{q} 2 \mathrm{w}$ vs placebo for IGA in patients without AR (not statistically significant). Safety in the overall population was consistent with the known dupilumab safety profile in adults and adolescents.

Conclusions: Dupilumab with concomitant TCS was equally efficacious in improving signs and symptoms of severe AD in children aged 6-11 years with and without comorbid AR. 


\section{P5.3}

\section{Dupilumab improves signs and symptoms of severe atopic dermatitis in children aged 6-11 years with and without comorbid asthma}

\section{Boguniewicz', ${ }^{1,}$ A. Wollenberg ${ }^{3}$, L. Sher ${ }^{4}$, E. Siegfried ${ }^{5}$, M. Bastian ${ }^{6}$, Z. Chen ${ }^{7}$, B. Shumel ${ }^{7}$}

${ }^{1}$ National Jewish Health, Denver, CO, USA;

2University of Colorado School of Medicine, Denver, CO, USA;

${ }^{3}$ Ludwig-Maximilian University, Munich, Germany;

${ }^{4}$ Northwestern University Feinberg School of Medicine, Chicago, IL, USA;

5Saint Louis University and Cardinal Glennon Children's Hospital, St. Louis, MO, USA;

${ }^{6}$ Sanofi, Frankfurt, Germany;

${ }^{7}$ Regeneron Pharmaceuticals, Inc., Tarrytown, NY, USA

Background: Atopic dermatitis (AD) is a chronic inflammatory skin disease that frequently occurs with other type 2 inflammatory diseases, including asthma. Dupilumab inhibits interleukin (IL)-4 and IL-13 signaling and is approved for treating multiple type 2 inflammatory diseases, including AD and asthma. Here, we evaluate the efficacy of dupilumab with concomitant topical corticosteroids (TCS) for severe AD in children with and without comorbid asthma.

Methods: In this double-blind, 16-week, phase 3 trial (NCT03345914), 367 children aged 6-11 years were randomized 1:1:1 to 300 mg dupilumab every 4 weeks (q4w), weight-based 100/200 mg dupilumab every 2 weeks (q2w), or placebo, with concomitant medium-potency TCS. Asthma history was ascertained by caregiver report.

Results: At baseline, $48 \% / 52 \% / 49 \%$ patients in the q4w/q2w/placebo groups reported a history of asthma; $52 \% / 48 \% / 51 \%$ patients had no history of asthma. Baseline AD disease severity was comparable in both groups. At Week 16, in both subgroups, significantly more patients receiving dupilumab q4w/q2w vs placebo achieved Investigator's Global Assessment score 0/1 (with asthma:37.9\%/27.0\% vs $11.7 \%$; without asthma: $28.1 \% / 32.2 \%$ vs $11.1 \%$ ), $\geq 75 \%$ improvement in Eczema Area and Severity Index (with asthma:65.5\%/58.7\% vs $25.0 \%$; without asthma: $73.4 \% / 76.3 \%$ vs $28.6 \%$ ), and $\geq 4$-point reduction from baseline in Peak Pruritus Numerical Rating Scale (with asthma:48.3\%/50.8\% vs $8.5 \%$; without asthma:53.2\%/66.1\% vs $15.9 \%$ ); $P<0.05$ between the verum and placebo groups. Safety in the overall population was consistent with the known dupilumab safety profile observed in adults and adolescents.

Conclusions: Dupilumab with concomitant TCS was equally efficacious in improving severe $\mathrm{AD}$ signs and symptoms in children aged 6-11 years with and without a history of comorbid asthma.

\section{URTICARIA}

\section{P2.6}

Charakterisierung von skin homing-Rezeptoren auf zirkulierenden CD4+ T-Lymphozyten bei Patienten mit chronisch-spontaner Urtikaria

\section{S. C. Hermann' ${ }^{1}$, J. Seidel', J. Pickert', 2 , M. Rauber- Ellinghaus ${ }^{1}$, Y. Xiang ${ }^{3}$, S. Altrichter ${ }^{3,4}$, W. Pfützner ${ }^{1,2}$, C. Möbs ${ }^{1}$}

${ }^{1}$ Klinische \& Experimentelle Allergologie, Klinik für Dermatologie und Allergologie, Philipps-Universität Marburg, Marburg, Deutschland;

${ }^{2}$ Universitätsklinikum Gießen und Marburg, Klinik für Dermatologie und Allergologie, Allergie-Zentrum Hessen, Marburg, Deutschland;

${ }^{3}$ Abteilung für dermatologische Allergologie, Klinik Dermatologie und Allergologie, Charité - Universitätsmedizin Berlin, Berlin, Deutschland;

${ }^{4}$ Universitätsklinik für Dermatologie und Venerologie, Kepler Universitätsklinikum Linz, Österreich

Die chronisch-spontane Urtikaria (CSU) ist eine durch rezidivierende, juckende Quaddeln gekennzeichnete Hauterkrankung, deren Pathomechanismen und insbesondere das Ausmaß der T-Zell-Beteiligung an diesen Prozessen, bis heute nicht abschließend geklärt sind. Kürzlich konnten wir bei der Untersuchung verschiedener T-Zell-Subpopulationen anhand der Sekretion von Markerzytokinen mittels ELISPOT-Analyse zeigen, dass in den untersuchten CSU-Patienten $(n=59)$ signifikant weniger IFN- $\gamma-$, IL-17- und IL-10-sezernierende T-Zellen nach unspezifischer Aktivierung mit anti-CD3/CD28-Antikörpern zu detektieren waren als in der Kontrollgruppe gesunder Probanden $(n=10)$.

Da bekannt ist, dass sich neben Mastzellen in läsionaler und nicht-läsionaler Haut von CSU-Patienten auch vermehrt CD4+ Zellen im Vergleich zu Biopsien gesunder Kontrollen finden lassen, wurde in dieser Studie das Potential von T-Zellen in die Haut zu migrieren mittels der auf zirkulierenden T-Zellen exprimierten skin homing-Rezeptoren CCR4, CCR8, CCR10 und CLA durchflusszytometrisch analysiert.

Bisher konnten wir beobachten, dass die CSU-Patienten, deren Basophile anhand von Fc\&RI-Antikörpern stimulierbar waren, mit steigender Serumautoreaktivität eine eher Th2-gewichtete T-Zell-Antwort zeigten, wohingegen bei der Subgruppe mit Fc\&RI-nicht-reaktiven Patienten und zusätzlicher Basopenie ein Trend zu erhöhten Th17-Zellen beobachtet wurde. Zusätzlich dazu, wurde eine weitere Klassifikation nach dem CSU-Subtyp der Patienten, d.h. nach Typ I (autoallergisch) oder Typ IIb (autoimmun), vorgenommen. Anhand der skin homing-Rezeptoranalysen konnte nun gezeigt werden, dass es in Abhängigkeit vom individuellen Basophilen- bzw. CSU-Subtyp zu einer unterschiedlichen Ausprägung der Rezeptorexpression auf verschiedenen T-Zellsubpopulationen kommt. Damit scheint sich die Annahme zu bestätigen, dass einzelne T-Zellsubsets unterschiedlich stark in die verschiedenen pathophysiologischen Prozesse der CSU involviert sind. 


\section{BIOLOGICS}

\section{P6.1}

\section{Omalizumab bei IgE-vermittelter \\ Nahrungsmittelallergie: eine systematische \\ Übersicht und Meta-Analyse}

M. Worm ${ }^{1}$, T. Zuberbier', R. A. Wood ${ }^{2}$, C. BindslevJensen $^{3}$, A. Fiocchi ${ }^{4}$, S. Chinthrajah ${ }^{5,6}$, A. Deschildre ${ }^{7}$, M. M. F. Rivas ${ }^{8}$, A. Santos ${ }^{9}$ X. Jaumont ${ }^{10}$, P. Tassinari ${ }^{10}$

${ }^{1}$ Department of Dermatology and Allergy, Charité Universitätsmedizin, Berlin, Germany;

${ }^{2}$ Division of Allergy and Immunology, Department of Pediatrics, Johns Hopkins University School of Medicine, Baltimore, Maryland, USA;

${ }^{3}$ Odense Research Center for Anaphylaxis, Odense

University Hospital, Odense, Denmark;

${ }^{4}$ Allergy Division, Bambino Gesù Children's Hospital, Istituti di Ricovero e Cura a Carattere Scientifico, Rome, Italy; ${ }^{5}$ Sean N. Parker Center for Allergy and Asthma Research, Stanford University, Stanford, California;

USA;

${ }^{6}$ Division of Pulmonary and Critical Care Medicine, Division of Allergy, Immunology and Rheumatology, Stanford University, Stanford, California, USA;

${ }^{7} \mathrm{CHU}$ Lille, Pediatric Pulmonology and Allergy Department, Hôpital Jeanne de Flandre, Université Nord de France, Lille, France;

${ }^{8}$ Allergy Department, Hospital Clinico San Carlos, and IdISSC, Madrid, Spain;

'Department of Women and Children's Health (Paediatric Allergy), School of Life Course Sciences, Faculty of Life Sciences and Medicine, King's College London, London, UK; ${ }^{10}$ Novartis Pharma AG, Basel, Switzerland

Einleitung: Der Nahrungsmittelallergie (NA) liegt eine IgE -abhängige Reaktion gegenüber Proteinen in der Nahrung zugrunde. In dieser Analyse wurden publizierte Daten zur klinischen Wirksamkeit und der Lebensqualität (QoL) bei nahrungsmittelallergischen Patienten untersucht, die Omalizumab (OMA), einen Antikörper gegen humanes Immunglobulin E (IgE), entweder als Monotherapie oder additiv zur oralen Immuntherapie (OIT) bei IgE-vermittelter NA erhalten haben.

Methode: 868 Datensätze wurden aus Literaturdatenbanken und Registern für klinische Studien nach randomisierten Studien (RCTs), kontrollierten Studien (CCTs) und Beobachtungsstudien (BS) unter Anwendung der PRISMA-Richtlinien durchsucht. Mit 8 RCTs, 18 CCTs und 4 BS zu OMA bei Erwachsenen und Kindern mit IgE-vermittelter NA wurde eine Meta Analyse durchgeführt, mit Prä-OMA und Placebo als Komparatoren.

Ergebnisse: OMA als Monotherapie oder als Ergänzung zur OIT war signifikant $(P<0,01)$ assoziiert mit einer Steigerung der oralen Schwellenwerte für Erdnuss (RR 6,34; RR 25,00), Kuhmilch (RR, 1,35; RR 3,00), Kuhmilch- und Ei-Allergie (RR 16,40) nach 13 Wochen in RCT bzw. CCT und MultinahrungsmittelAllergie (RR 1,75; RR 18,18; RR 7,33) nach 8-16, 12, 17 und 36 Wochen in RCT, CCT bzw. BS. Weiterhin war OMA als Monotherapie oder zusätzlich zu OIT mit signifikanten $(P<0,01)$ Veränderungen der QoL verbunden, die anhand des FAQLQ (MD $-2,11$ ) und der päd. QoL-Inventory Skala (MD 26,72) nach 261 bzw. 17 Wochen in CCT bzw. BS ermittelt wurden.
Schlussfolgerung: Die Analyse zeigt, dass OMA in zahlreichen Studien bei Kindern und Erwachsenen zu einer verbesserten Verträglichkeit eines oder mehrerer Nahrungsmittel bei IgEvermittelter Allergie führt und dies einen signifikanten Einfluss auf die QoL der Patienten hat. Insbesondere für schwer betroffene Patienten bietet OMA aufgrund der bisher vorliegenden Daten eine Option, das Risiko einer anaphylaktischen Reaktion aufgrund von unbeabsichtigtem Allergenkontakt zu reduzieren.

\section{P6.2}

\section{Anhaltende Wirksamkeit und Sicherheit} von Omalizumab bei chronischer Rhinosinusitis mit Nasenpolypen: eine Open-LabelExtensionsstudie

C. Bachert ${ }^{1}$, P. Gevaert ${ }^{1}$, R. Saenz ${ }^{2}$, J. Corren ${ }^{3}$, J. Han ${ }^{4}$, J. Mullol' ${ }^{5}$, S. Lee ${ }^{6}$, R. Zhao' ${ }^{2}$, M. Howard ${ }^{7}$, K.Wong ${ }^{2}$, L. Islam ${ }^{8}$, M. Ligueros-Saylan ${ }^{9}$, T. Omachi

${ }^{1}$ Ghent University and Ghent University Hospital, Ghent, Belgium;

${ }^{2}$ Genentech, Inc., South San Francisco, CA, USA;

${ }^{3}$ David Geffen School of Medicine at UCLA, Los Angeles, CA, USA;

${ }^{4}$ Eastern Virginia Medical School, Norfolk, VA, USA;

5Universitat de Barcelona, CIBERES, Barcelona, Catalonia,

Spain;

'University of Pittsburgh, PA, USA;

${ }^{7}$ Roche, Mississauga, ON, Canada;

${ }^{8}$ Roche, Welwyn Garden City, UK;

${ }^{9}$ Novartis Pharmaceuticals Corporation, East Hanover, NJ, USA

Einleitung: Diese offene Phase-III-Extensionsstudie (OLE) untersuchte die anhaltende Wirksamkeit und Sicherheit sowie die Persistenz des Ansprechens auf Omalizumab (OMA) bei Erwachsenen mit chronischer Rhinosinusitis mit Nasenpolypen (CRSwNP) welche unzureichend auf intranasale Kortikosteroide ansprechen und die zuvor an den randomisierten, doppelblinden, placebokontrollierten Phase-III-Studien mit OMA (POLYP1 oder POLYP2) teilgenommen hatten.

Methode: Erwachsene Patienten $(n=249)$, die während POLYP1/2 24 Wochen lang OMA oder Placebo (PBO) erhielten, nahmen an OLE teil. Die Behandlungsdauer betrug 28 Wochen mit OMA, gefolgt von einer 24-wöchigen Nachbeobachtungszeit ohne OMA. Deskriptive Erfassung der Veränderung des Nasenpolypenscores (NPS), des nasalen Kongestionsscores (NCS) und des Sino-Nasal Outcome Test-22 (SNOT-22) gegenüber dem Ausgangswert während der Studie. Das Auftreten von unerwünschten Ereignissen (AEs) allgemein und solcher, die zum Absetzen von OMA führten.

Ergebnisse: Während des OLE-Behandlungszeitraums kam es bei den Patienten, die OMA in POLYP1/2 erhielten, bis
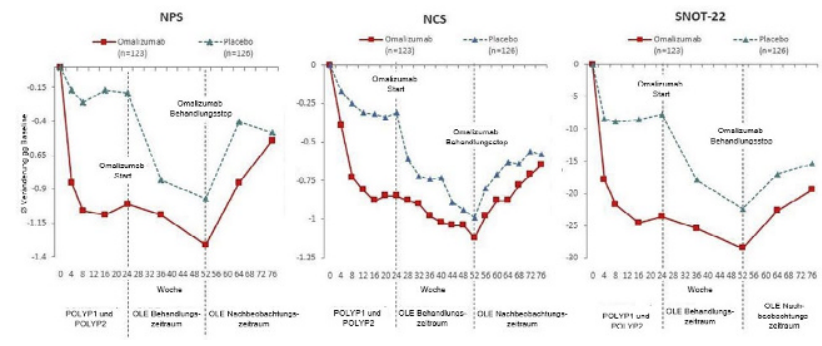
Woche $52 \mathrm{zu}$ anhaltenden Verbesserungen des NPS, NCS und SNOT-22, während bei den Patienten, die PBO in POLYP1/2 erhielten, rasche und klinisch relevante Verbesserungen auftraten. Die Werte verschlechterten sich allmählich nach Absetzen von OMA, blieben aber in Woche 76 unter den Werten vor der Behandlung (Abbildung). Das Sicherheitsprofil war ähnlich wie in früheren Berichten (Gevaert et al. JACI. 2020).

Schlussfolgerung: Das Sicherheits- und Wirksamkeitsprofil aus dieser Studie unterstützt die Fortsetzung der OMA-Behandlung bei Patienten mit CRSwNP. Bei den Patienten, die während der Doppelblindphase OMA erhielten, wurden bis Woche 52 anhaltende Verbesserungen beobachtet. Patienten, die während der Doppelblindphase PBO erhielten, zeigten während der OLE Studie ein ähnlich günstiges Ansprechen wie die Patienten in den POLYP1/2 Studien.

\section{P6.3}

\section{Dupilumab provides early and durable}

improvement of symptoms in patients with chronic rhinosinusitis with nasal polyps: results from the SINUS-24 and SINUS-52 phase 3 trials

P. Gevaert', S. E. Lee ${ }^{2}$, R. Settipane ${ }^{3}$, M. Wagenmann ${ }^{4}$, J. Msihid ${ }^{5}$, S. Siddiqui ${ }^{6}$, S. Nash ${ }^{6}$, J. A. Jacob-Nara ${ }^{7}$, A. H. Khan ${ }^{5}$, S. Kamat ${ }^{6}$, C.-C. Chuang ${ }^{8}$

${ }^{1}$ Ghent University, Ghent, Belgium;

${ }^{2}$ Department of Otolaryngology-Head \& Neck Surgery, University of Pittsburgh Medical Center, Pittsburgh, PA, USA;

${ }^{3}$ The Warren Alpert Medical School of Brown University, Providence, RI, USA;

${ }^{4}$ Department of Otorhinolaryngology, Düsseldorf University Hospital (UKD), Düsseldorf, Germany;

${ }^{5}$ Sanofi, Chilly-Mazarin, France;

${ }^{6}$ Regeneron Pharmaceuticals, Inc., Tarrytown, NY, USA;

${ }^{7}$ Sanofi, Bridgewater, NJ, USA;

${ }^{8}$ Sanofi, Cambridge, MA, USA

Rationale: Chronic rhinosinusitis with nasal polyps (CRSwNP) is a type 2 inflammatory disease, with cardinal symptoms of nasal congestion (NC), loss of smell (LoS), and rhinorrhea significantly impacting patients' daily lives. We report dupilumab's effect on patient-reported CRSwNP symptoms from SINUS-24 (NCT02912468) and SINUS-52 (NCT02898454) studies.

Methods: Post-hoc analysis in CRSwNP patients receiving dupilumab $300 \mathrm{mg}$ or placebo q2w. Pooled SINUS-24/52 intentto-treat (ITT) population $(N=724)$ was used for assessment to Week 24 and SINUS-52 population $(N=303)$ for Week 52 assessment. Patients reported symptom scores $(0=$ none; $1=$ mild; $2=$ moderate; $3=$ severe) daily for NC, LoS, and rhinorrhea. Proportions of patients with baseline scores $\geq 2$ (moderate/severe) achieving improvement (score $\leq 1$; none/mild) at Weeks 2, 24, 52 were reported for the ITT population and subgroups with asthma/prior sinonasal surgery.

Results: At baseline, $86.7 \%, 94.1 \%$, and $64.1 \%$ of patients had scores $\geq 2$ for NC, LoS, and rhinorrhea, respectively. Significantly more patients achieved improvement (score $\leq 1$; none/mild symptoms) with dupilumab vs placebo at Weeks 2/24/52 (dupilumab vs placebo, NC: $11.5 \% / 54.2 \% / 54.6 \%$ vs $\quad 1.6 \% / 13.7 \% / 15.9 \%$; LoS, $5.1 \% / 43.4 \% / 43.8 \%$ vs $1.1 \% / 5.5 \% / 4.2 \%$; rhinorrhea, $9.4 \% / 53.2 \% / 58.3 \%$ vs $2.2 \% / 15.6 \% / 20.4 \%$ ). Results were consistent across subgroups at Weeks 24 and 52. Among patients not achieving scores $\leq 1$ at Week 24, more dupilumab vs placebo-treated patients experienced symptom improvement (scores of $>1-\leq 2$ ).

Conclusions: In CRSwNP patients with moderate-to-severe symptom burden at baseline, dupilumab significantly improved patient-reported symptoms vs placebo. Symptom improvement was observed as early as Week 2 , and continued to Week 52 , suggesting early onset, durable treatment effect of dupilumab.

\section{P6.4}

\section{Dupilumab improves outcomes of concurrent asthma and chronic sino-nasal conditions in patients with atopic dermatitis - a pooled analysis of four phase 3 Studies: LIBERTY AD SOLO 1 \& 2, CHRONOS, and CAFÉ}

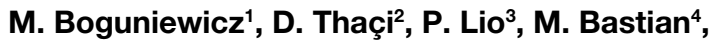 Z. Chen ${ }^{5}$, M. Ardeleanu ${ }^{5}$}

${ }^{1}$ National Jewish Health and University of Colorado School of Medicine, Denver, CO, USA;

${ }^{2}$ Comprehensive Centre for Inflammation Medicine, University Hospital Schleswig-Holstein, Campus Lübeck, Lübeck, Germany;

${ }^{3}$ Feinberg School of Medicine, Northwestern University, Chicago, IL, USA;

${ }^{4}$ Sanofi, Frankfurt, Germany;

${ }^{5}$ Regeneron Pharmaceuticals, Inc., Tarrytown, NY, USA

Background: Dupilumab, a fully human anti-interleukin (IL)-4 receptor $\alpha$ monoclonal antibody, inhibits signaling of IL-4 and IL-13, key drivers of type 2-mediated inflammation. Dupilumab is approved for treatment of moderate-to-severe atopic dermatitis $(\mathrm{AD})$ in adults and adolescents and severe $\mathrm{AD}$ in children. Here, we assessed dupilumab's efficacy in adults with concurrent $\mathrm{AD}$, asthma, and chronic nasal/paranasal sinus conditions (N/PNS) from 4 phase 3 trials: LIBERTY AD SOLO 1 \& 2 (NCT02277743, NCT02277769), CHRONOS (NCT02260986), and CAFÉ (NCT02755649).

Methods: Data were pooled from adult patients with moderate-to-severe $\mathrm{AD}$, asthma requiring treatment plus baseline Asthma Control Questionnaire (ACQ-5) score $\geq 0.5$, and N/PNS (12.7\% of study patients). Patients received subcutaneous dupilumab $300 \mathrm{mg}$ every 2 weeks (q2w; $n=95$ ) or weekly (qw; $n=102)$ or placebo $(n=114)$. Outcomes were assessed at Week 16: for asthma, by ACQ-5; for N/PNS, by 22-item SinoNasal Outcome Test (SNOT-22); and for AD, by Eczema Area and Severity Index (EASI) and peak pruritus Numerical Rating Scale (NRS). Safety was assessed.

Results: Baseline characteristics were consistent with those of the overall study populations. Dupilumab q2w/qw vs placebo improved scores (presented as least squares mean reduction/\% reduction from baseline) on the ACQ-5 (0.53/0.66 vs $0.24 ; \mathrm{P}=0.0242 / 0.0007)$, SNOT-22 $(12.27 / 13.39$ vs 6.47 ; $P=0.0110 / 0.0017)$, EASI $(77.7 \% / 75.3 \%$ vs $35.5 \% ; P<0.0001)$, and peak pruritus NRS $(46.1 \% / 50.7 \%$ vs $24 \% ; P<0.0001)$. Injection-site reactions and conjunctivitis were more common in dupilumab-treated than placebo-treated patients.

Conclusion: This subgroup analysis shows that dupilumabtreated AD patients with asthma and N/PNS experienced clinically and statistically significant improvement in all 3 diseases. 
P6.5

Dupilumab shows sustained efficacy in patients with asthma receiving high-dose inhaled corticosteroids at baseline: The Liberty Asthma Traverse Study

I. D Pavord1', A. Bourdin², A. Papi ${ }^{3}$, C. Domingo4, J. Corren ${ }^{5}, \mathrm{X} . \mathrm{Mao}^{6}$, B. Ortiz ${ }^{7}, \mathrm{M}$. Djandji $^{8}, \mathrm{M}$. Ruddy $^{7}$, E. Laws ${ }^{6}$, N. Amin', D. J. Lederer ${ }^{7}$, M. Hardin ${ }^{8}$

${ }^{1}$ NIHR Oxford Biomedical Research Centre, University of Oxford, Oxford, UK;

2University of Montpellier, Montpellier, France;

${ }^{3}$ University of Ferrara, S. Anna University Hospital, Ferrara, Italy;

${ }^{4}$ Corporació Sanitària Parc Taulí, Sabadell, Autonomous University of Barcelona, Barcelona, Spain;

${ }^{5}$ David Geffen School of Medicine at UCLA, Los Angeles, CA, USA;

${ }^{6}$ Sanofi, Bridgewater, NJ, USA;

${ }^{7}$ Regeneron Pharmaceuticals, Inc., Tarrytown, NY, USA;

${ }^{8}$ Sanofi, Cambridge, MA, USA

Background: Dupilumab (DPL), a fully human monoclonal antibody, blocks the shared receptor component for interleukin-4 and -13, key and central drivers of type 2 inflammation. TRAVERSE (NCT02134028), a single-arm, open-label extension (OLE) study, evaluated long-term safety and tolerability of DPL in patients (pts) aged $\geq 12$ years with uncontrolled, moderate-to-severe asthma who participated in a previous DPL asthma study, including pts from the phase 2b DRI study (P2B; NCT01854047) and the phase 3 QUEST study (NCT02414854). In both parent studies, add-on DPL 200/300 mg every 2 weeks (q2w) vs placebo (PBO) significantly reduced severe asthma exacerbations and improved pre-bronchodilator (BD) FEV1. We evaluated long-term efficacy of DPL in pts on medium- and high-dose inhaled corticosteroids (ICS) at parent study baseline (PSBL).

Methods: TRAVERSE pts received add-on DPL $300 \mathrm{mg}$ q2w for up to 96 weeks. Pts were stratified by ICS use (high or medium) at PSBL. Unadjusted annualized severe exacerbation rates (AERs) and change from PSBL in pre-BD FEV1 over time were analyzed in each group.

Results: 2,062 pts from QUEST $(n=1,530 ;$ PBO/DPL: 517, DPL/DPL: 1,013$)$ and P2B $(n=532 ;$ PBO/DPL: 111 , DPL/DPL: 421) rolled over into the OLE. $54.7 \%$ (PBO/DPL: $56.5 \%$, DPL/DPL: $53.8 \%$ ) of QUEST and $52.6 \%$ (PBO/DPL: $50.5 \%$, DPL/DPL: $54.7 \%$ ) of P2B pts were on highdose- ICS at PSBL. Over 96 weeks of the OLE, AERs seen in parent studies decreased in PBO/DPL pts and remained low for DPL/DPL pts on both medium- and high-dose ICS (medium: 0.187-0.266; high: 0.388-0.449). Pre-BD FEV1 improved in both subgroups for PBO/DPL pts and was sustained for DPL/DPL pts (Week 48: medium: 0.26-0.38L, high: 0.25-0.34L; Week 96: medium: 0.26$0.31 \mathrm{~L}$, high: $0.19-0.35 \mathrm{~L})$. DPL's safety profile during the OLE was consistent with parent studies.

Conclusions: In line with parent studies, DPL showed sustained efficacy up to 3 years in pts on medium- and high-dose ICS with uncontrolled, moderate-to-severe asthma.

\section{P6.6}

\section{Switch in der Biologika-Therapie bei schwerem} Asthma bronchiale - Erfahrungen bei Kindern

\section{W. G. van Kessel' ${ }^{1}$, N. Fischedick ${ }^{1}$, F. Brinkmann ${ }^{2}$,} A. Wiemers' ${ }^{2}$, S. Flümann', C. Koerner-Rettberg'

${ }^{1}$ Klinik für Kinder- und Jugendmedizin, Marien-Hospital Wesel, Wesel, Deutschland;

${ }^{2}$ Klinik für Kinder- und Jugendmedizin, Josef-Hospital Bochum, Klinikum der Ruhr-Universität Bochum, Bochum, Deutschland

Hintergrund: Zur Therapie des schweren Asthmas im Kindesalter hat sich die Palette der zur Verfügung stehenden Biologika in den letzten Jahren erweitert: Nach Zulassung von Omalizumab in Deutschland ab 12 J. 2005 und ab 6 J. 2009 wurde Mepolizumab 2018 ab 6 J., und Dupilumab 2019 ab 12 J. zugelassen. Die Wirkstoffe unterscheiden sich bzgl. immunologischem Angriffsort und Phänotyp-Zulassungskriterien, mit jedoch signifikanter Überlappung. Eine differenzierte Biologikum-Wahl stellt somit eine neue Herausforderung für Behandler dar und profitiert von Erfahrungen an Patienten mit stattgehabtem Biologikum-Wechsel.

Methodik: Es werden für 8 Patienten im Alter von 9-17 Jahren mit schwerem unkontrolliertem Asthma Erfahrungen zum Biologika-Wechsel berichtet, indem der individuelle AsthmaPhänotyp, Biologika-Primärtherapie, Auswahl-Grund, Biologika-Zweittherapie, Wechselgrund, sowie individuelle Verträglichkeit und Effektivität der Biologika dargestellt werden.

Ergebnisse: Gründe für eine Primärtherapie mit einer retrospektiv unzureichend wirksamen Substanz waren in 5 von 8 Fällen eine Konstellation außerhalb der Zulassung des später eingesetzten effektiven Biologikums (Alter, IgE-Höhe oder fehlende perenniale Sensibilisierung). Weitere Wechselgründe waren Nebenwirkungen, die alle drei in der Pädiatrie zugelassenen Biologika betrafen.

Schlussfolgerung: Omalizumab und Dupilumab waren Mepolizumab bzgl. Effektivität beim Asthma-Phänotyp mit Eosinophilie und gleichzeitiger Atopie überlegen, und die unzureichende Mepolizumab-Effektivität war in diesen Fällen ein Wechselgrund zu einem anderen Biologikum. Dagegen zeigte ein Anti-IL-5-Wirkstoff (Mepolizumab oder Benralizumab) bei 2 Patienten mit schwerem isoliert-eosinophilem (nicht atopischem) Asthma eine sehr gute Wirksamkeit, was die Wichtigkeit der genauen Endotyp-Passung unterstreicht, ggf. unter Inkaufnahme von off-label-Einsätzen. Verträglichkeit kann selten ein Wechselgrund sein. 


\section{Baseline-Charakteristika von Patienten} mit schwerem Asthma unter Dupilumab in der klinischen Praxis, in einer multinationalen, nicht interventionellen, Real-World-Studie (ProVENT)

\section{Lommatzsch', S. Korn², O. Schmidt', H. Timmermann ${ }^{4}$, H. Watz ${ }^{5}$, A. Radwan ${ }^{6}$, L. De Prado- Gómez $^{7}$, A. Atenhan ${ }^{8}$, S. Barbus ${ }^{9}$, M. Thakur ${ }^{8}$}

'Universität Rostock, Rostock, Deutschland;

${ }^{2}$ Clinical Research Centre Respiratory Diseases IKF Pneumologie $\mathrm{GmbH}$ \& Co. KG, Mainz, Deutschland; ${ }^{3}$ Lungen- und Bronchialheilkunde, Koblenz, Deutschland; ${ }^{4}$ Allergologie, Lungen- und Bronchialheilkunde, Hamburg, Deutschland;

${ }^{5}$ Pneumologische Forschungsinstitut an der LungenClinic Grosshansdorf, Deutsches Zentrum für Lungenforschung (DZL), Grosshansdorf, Germany;

${ }^{6}$ Regeneron Pharmaceuticals, Inc. Tarrytown, NY, USA;

${ }^{7}$ Sanofi, Reading, Großbritannien;

${ }^{8}$ Sanofi, Berlin, Deutschland;

${ }^{9}$ Sanofi, Frankfurt, Deutschland

Einleitung: Dupilumab, ein humaner monoklonaler Antikörper, blockiert die gemeinsame Rezeptorkomponente von IL-4 und IL-13, welche die Schlüsselmediatoren Typ-2 vermittelter Erkrankungen sind. In der EU ist Dupilumab zur Behandlung von schwerem unkontrolliertem Asthma zugelassen, jedoch ist über die Charakteristika von Patienten, die Dupilumab erhalten, wenig bekannt. ProVENT ist eine nicht-interventionelle, Real-World-Langzeit-Studie in Deutschland, der Schweiz und Österreich.

Ziel: Die Erhebung der Baseline-Charateristika von Pat. mit Typ-2-Asthma unter Dupilumab-Therapie, einschließlich demographischer Daten, Asthmakontrolle, Vormedikation, Typ-2-Komorbiditäten und Biomarker-Expression (Bluteosinophile [Eos], Fraktion des exhalierten Stickstoffmonoxids [FeNO], und IgE).

Ergebnisse: Baseline-Charakteristika von 99 derzeit in ProVENT registrierten Pat. werden in einer Tabelle dargestellt. Es handelt sich mehrheitlich um Frauen. Die meisten Patienten haben eine schlechte Asthma-Kontrolle und Lebensqualität, $15 \%$ der Patienten berichten über eine Prednisolondauertherapie. Die Mehrheit weist deutlich erhöhte Typ-2-Biomarker auf, und über $60 \%$ haben Typ-2-Komorbiditäten. Es werden sowohl Pat. mit allergischen als auch intrinsischem Asthma behandelt.

Fazit: Die Mehrheit der Dupilumab-Patienten, welche an dieser Real-World-Studie teilnehmen, weist bei Therapiebeginn erhöhte Typ-2-Biomarker, hohe Exazerbationsraten und eine schlechte Asthma-Kontrolle und Lebensqualität auf. Dupilumab ist meist das erste bei den Pat. eingesetzte Biologikum.
Dupilumab efficacy in children with uncontrolled, moderate-to-severe type 2 asthma, with and without evidence of allergy, in the phase 3 VOYAGE study

\section{N. G. Papadopoulos ${ }^{1,2}$, S. J. Szefler ${ }^{3}$, L. B. Bacharier ${ }^{4}$, J.F. Maspero ${ }^{5}$, C. Domingo ${ }^{6}$, N. Daizadeh ${ }^{7}$, D. J Lederer $^{8}$, M. Hardin 7 , J. A. Jacob-Nara ${ }^{7,9}$, Y. Deniz ${ }^{8}$, R. Gall ${ }^{8}$, B. Ortiz ${ }^{8}$, M. Djandji ${ }^{7}$, P.J. Rowe ${ }^{9}$}

${ }^{1}$ Royal Manchester Children's Hospital, Manchester, UK; 22nd Pediatric Clinic, University of Athens, Greece; ${ }^{3}$ University of Colorado School of Medicine, Aurora, CO, USA;

${ }^{4}$ Monroe Carell Jr Children's Hospital at Vanderbilt University Medical Center, Nashville, TN, USA;

${ }^{5}$ Fundación CIDEA, Buenos Aires, Argentina;

${ }^{6}$ Corporació Sanitària Parc Taulí, Sabadell, Autonomous University of Barcelona, Barcelona, Spain;

${ }^{7}$ Sanofi, Cambridge, MA, USA;

${ }^{8}$ Regeneron Pharmaceuticals, Inc., Tarrytown, NY, USA; 'Sanofi, Bridgewater, NJ, USA

Background: Dupilumab (DPL), a fully human monoclonal antibody, blocks the shared receptor component for interleukin-4/13, key and central drivers of type 2 inflammation. In the 52-week, phase 3 VOYAGE study (NCT02948959), add-on DPL $100 / 200 \mathrm{mg}$ (body weight $\leq 30 />30 \mathrm{~kg}$ ) every 2 weeks vs placebo (PBO) reduced severe asthma exacerbations and improved prebronchodilator (BD)\% predicted FEV1 (FEVlpp) at Week (Wk) 12 in children aged 6 to $<12$ years with uncontrolled, moderateto-severe, type 2 asthma ( $\geq 150$ blood eosinophils/ $\mu$ l or FeNO $\geq 20 \mathrm{ppb}$ at baseline [BL]). We evaluated DPL efficacy in pediatric patients (pts) with type 2 asthma with/without evidence of allergic asthma phenotype (serum total $\mathrm{IgE} \geq 30 \mathrm{IU} / \mathrm{mL}$ and $\geq 1$ perennial aeroallergen-specific IgE $\geq 0.35 \mathrm{kU} / \mathrm{L}$ at baseline).

Methods: Annualized severe asthma exacerbation rate (AER) was assessed post hoc using a negative binomial model. Change from baseline in pre-BD FEV1 and pre-BD FEV1pp was assessed using mixed-effect models with repeated measures.

Results: VOYAGE enrolled 350 pts with type 2 asthma, 261 with and 89 without evidence of allergic phenotype. BL demographics were similar; type 2 biomarker levels (blood eosinophils, FeNO, serum total IgE) and ongoing atopic comorbidities were higher in pts with vs without evidence of allergic phenotype. DPL vs PBO significantly reduced AER, irrespective of allergic phenotype. Changes from $\mathrm{BL}$ to $\mathrm{Wk} 12$ and 52 in preBD FEVlpp and FEV1 were greater in DPL- vs PBO-treated pts in both subgroups, irrespective of allergic phenotype. In the safety population, TEAE incidence was similar across treatment groups; the most common TEAE occurring more frequently in the DPL group was injection-site erythema (DPL: $12.9 \%$; PBO: $9.7 \%)$.

Conclusions: A high proportion of pediatric pts with type 2 inflammatory asthma enrolled in VOYAGE had evidence of an allergic asthma phenotype. DPL reduced asthma exacerbations in pts with/without evidence of allergic asthma. 
P6.9

Complete symptom control in chronic spontaneous urticaria patients leads to improves sleep and quality of life: results from the phase $2 b$ ligelizumab study

\section{A. Gimenez-Arnau', M. Maurer ${ }^{2}$, J. Bernstein ${ }^{3}$, N. Barbier ${ }^{4}$, E. Hua ${ }^{4}$, T. Severin 4 , R. Janocha ${ }^{4}$, M.-M. Balp ${ }^{4}$}

'University Autonoma, Barcelona, Spain; ${ }^{2}$ Charite - Universitätsmedizin Berlin, Berlin, Germany; ${ }^{3}$ University of Cincinnati, Cincinnati, USA; ${ }^{4}$ Novartis Pharma AG, Basel, Switzerland

Introduction: Chronic spontaneous urticaria (CSU) symptoms can adversely affect daily activities, sleep and cause substantial health-related quality of life (HRQoL) impairment. Here, we analysed the impact of urticaria activity (UAS7) on Weekly Sleep Interference (WSI) and Dermatology Life Quality Index (DLQI), during a 20-week Phase 2b core study (NCT02477332) and a 52-week open-label, single-arm Phase $2 \mathrm{~b}$ extension study (NCT02649218)

Methods: In the Phase 2b 20-week core study, adult patients with moderate to severe CSU (defined by [UAS7] $\geq 16$ ) were randomised to receive ligelizumab 72 or $240 \mathrm{mg}$, omalizumab $300 \mathrm{mg}$ or placebo every 4 weeks for five injections. Following a 16 -week washout period after the last dose in the core study, eligible patients (UAS7 $\geq 12$ ) entered a 52 -week open-label, singlearm (ligelizumab $240 \mathrm{mg} \mathrm{q} 4 \mathrm{w}$ ) extension study.

Results: From the core study population, $70.6 \%$ (226/320) of patients that completed the treatment-free follow-up period, were eligible based on their disease activity score (UAS7 $\geq 12$ ) after the washout period. In total, $88.9 \%$ of these patients $(201 / 226)$ completed the extension study. The comparison by UAS7 responder status demonstrated that urticaria-free patients had a significantly lower least square mean \pm SD, WSI score vs. patients with well-controlled urticaria in both core $(0.2 \pm 0.2 \mathrm{vs}$. $1.0 \pm 0.2)$ and extension $(0.3 \pm 0.2$ vs. $0.7 \pm 0.2)$ studies. WSI and DLQI scores were highly correlated with disease activity (UAS7 scores); and reduced with decreased disease activity.

Conclusions: Improvement in urticaria activity is associated with improvement in sleep and dermatology-related QoL. Overall, effective treatments such as ligelizumab have benefits beyond urticaria symptoms, showing the importance of targeting complete urticaria control. Patients free of urticaria are more likely to achieve a low DLQI score (low impact on QoL) and low WSI score (low impact on sleep).

\section{P6.10}

\section{Ligelizumab ist effektiver in Patienten}

mit chronischer spontaner Urtikaria welche vorher mit Omalizumab behandelt wurden

\section{K. Sitz' ${ }^{1}$, G. Sussmann²}

1 University of Arkansas, Little Rock, USA;

${ }^{2}$ University of Toronto, Toronto, Canada

Rationale: In der Phase-2b-Kernstudie (NCT02477332) zeigte Ligelizumab ( $72 \mathrm{mg}, 240 \mathrm{mg}$ alle vier Wochen [q4w]) eine frühere und stärkere Verbesserung der Urtikaria-Krankheitsak- tivität bis Woche $20 \mathrm{im}$ Vergleich zu Omalizumab (300 mg q4w) und Placebo. Hier wurde die Wirkung von Ligelizumab auf die Krankheitsaktivität der Urtikaria bei CSU-Patienten untersucht, die zuvor mit Omalizumab behandelt wurden.

Methoden: Wir analysierten die Wirkung der Behandlung auf die Krankheitsaktivität (mittels UAS7) bei Patienten, die in der 20-wöchigen Phase-2b-Kernstudie mit Omalizumab $300 \mathrm{mg}$ behandelt wurden. An die Behandlung mit Omalizumab folgte eine bis zu 24-wöchigen behandlungsfreien Auswaschphase. Anschließend wurden die Patienten in der einarmigen, 52-wöchigen Phase-2b-Open-Label-Erweiterungsstudie auf Ligelizumab $240 \mathrm{mg} \mathrm{q} 4 \mathrm{w}$ umgestellt.

Ergebnisse: Zu Beginn der Extensionsstudie war der mittlere UAS7-Werte \pm SD der 53 Patienten, die von Omalizumab auf Ligelizumab umgestellt wurden, zwischen der Kernstudie $(29,7 \pm 8,0)$ und in der Erweiterungsstudie $(27,8 \pm 8,9)$ vergleichbar. Die UAS7-Werte nach der Behandlung mit Ligelizumab waren niedriger, und die Veränderung gegenüber dem Ausgangswert (CFB) in UAS7 war bei der Behandlung mit Ligelizumab größer als bei der Behandlung mit Omalizumab in Woche 12 (Ligelizumab: $6.9 \pm 9.3$ und $-20.9 \pm 13.1$ versus Omalizumab: $11.8 \pm 13.3$ und $-17.7 \pm 13.1$ ) und Woche 20 (Ligelizumab: $6,2 \pm 9,4$ und $-21,7 \pm 12,3$ versus Omalizumab: $12,4 \pm 13,0$ und $-17,1 \pm 12,6)$. Der Rückgang von UAS7 blieb unter der Behandlung mit Ligelizumab während der gesamten 52-wöchigen Verlängerungsstudie erhalten (Woche $28=4,6 \pm 9,2$; Woche $36=3,8 \pm 8,3$, Woche $44=4,4 \pm 9,2$ und Woche $52=4,7 \pm 9,1$ ).

Fazit: Bei Patienten mit CSU, die zuvor mit Omalizumab behandelt wurden, kann Ligelizumab einen zusätzlichen Nutzen in Form einer weiteren Verringerung der Urtikaria-Aktivität bieten

\section{INSECT VENOM ALLERGY}

\section{P7.1}

\section{Mangelhafte Aufklärung und Handhabung des Notfallsets bei Insektengiftallergikern}

\section{J. Zarnowski, J.-C. Simon, R. Treudler}

Klinik für Dermatologie, Venerologie und Allergologie, Universitätsklinikum Leipzig

Hintergrund: Insektengifte sind die häufigsten Auslöser anaphylaktischer Reaktionen bei Erwachsenen. Unser Ziel war es das Wissen der Betroffenen in Hinblick auf die Anwendung des Notfallsets zu überprüfen.

Methoden: Insektengiftallergiker, die bei Erstvorstellung in unserer Allergiesprechstunde im Besitz eines Notfallsets waren, wurden mittels intern konzipiertem Fragebogens bezüglich der Aufklärung über ihre Notfallmedikamente befragt. Zudem wurden die Teilnehmer um eine praktische Demonstration des Adrenalinautoinjektors (AAI) gebeten.

Ergebnisse: Wir haben 55 Patienten in unsere Befragung eingeschlossen (35 Frauen; durchschnittliches Alter: $54.6 \pm 17.5$ Jahre). Die Hymenopterengiftallergie (Wespe: $87.3 \%$; Hornisse: $9.1 \%$; Biene: $3.6 \%$ ) war bei $9.1 \%$ vom Schweregrad Müller $\mathrm{I}^{\circ}$, bei je $25.5 \% \mathrm{II}^{\circ}$ oder $\mathrm{IV}^{\circ}$ und bei $38.2 \% \mathrm{III}^{\circ}$. $85.5 \%$ berichteten, über die Anwendung der Notfallmedikamente mündlich aufgeklärt worden zu sein aber lediglich $37.7 \%$ erhielten eine Einweisung in die praktische Anwendung des AAI. 54.5\% hatten ihr Notfallset während der Vorstellung nicht dabei und nur bei $30.9 \%(n=17 / 55)$ konnte eine fehlerfreie 
Handhabung ihres Notfallsets beobachtet werden. Die häufigsten Fehlerquellen zeigten sich bei der praktischen Anwendung des AAI (8/38; $21.1 \%)$, dem Zeitpunkt der Medikamenteneinnahme (10/38; $26.3 \%)$ oder beiden zusammen (20/38; $52.6 \%)$. Bei $35.7 \%$ wurde hierbei eine Fehlinjektion des Demonstrationsinjektors in den Finger beobachtet. Zudem gaben $20.0 \%$ an, den AAI unmittelbar nach jedem Stichereignis unabhängig von den klinischen Beschwerden anwenden zu wollen.

Schlussfolgerung: Unsere Daten demonstrieren einen erheblichen Mangel in Aufklärung und Handhabung des Notfallsets sowie eine hohe Rate an Fehlinjektionen, sodass Verschreibende eines Notfallsets vermehrt für die Notwendigkeit einer ausreichenden Aufklärung sensibilisiert werden sollten.

\section{FOOD ALLERGY}

\section{P7.2}

\section{Dermatologie meets Gastroenterologie: eine typische Weizenallergie?}

\section{H. Adib-Tezer, R. Kiesslich, C. Bayerl}

Helios Dr. Horst Schmidt Kliniken, Wiesbaden, Deutschland

Hintergrund: Wir berichten über eine 32-jährige Patientin, die seit 3 Jahren an krampfartigen Schmerzen retrosternal und im Oberbauch litt. Diese traten ca. 4-5 Stunden nach der Nahrungsaufnahme auf. Bei Verdacht auf eine eosinophile Ösophagitis erfolgte eine Therapie mit Jorveza ${ }^{\circ} 0,5 \mathrm{mg} 2 \mathrm{x} / \mathrm{d}$ ohne relevante Symptomreduktion. Eine weizenreduzierte Diät führte zu einer deutlichen Beschwerdebesserung.

Material und Methoden: Es erfolgte eine Labordiagnostik mit ges.-IgE, sIgE, Tryptase. In vivo wurden Pricktestungen mit Nahrungsmittelextrakten, mit Aeroallergenen, eine Prick-toPricktestung mit nativen Nahrungsmitteln und eine konfokale Laser-Endomikroskopie (CLE), Fa. Cellvizio ${ }^{\circ}$, zur intestinalen Nahrungsmittelprovokation durchgeführt.

Ergebnisse: Es fand sich eine CAP-Klasse $1(0,56 \mathrm{kU} / \mathrm{l})$ für Weizenmehl, eine CAP-Klasse 0 für rTri a 19 aus Weizen, alphaGal aus Rind, Hühnerweiweiß und -eigelb, nGal d1, nGal d2 aus Hühnerei sowie für nBos d4, nBos d5 und nBos d8 aus Kuhmilch. Des Weiteren fanden sich in den Pricktestungen Soforttypreaktionen gegenüber Weizenmehl, Eigelb sowie gegenüber Frühblüher- und Gräserpollen. Schließlich zeigte die CLE eine positive Reaktion auf Weizensuspension.

Schlussfolgerung: Eine klassische Weizenallergie manifestiert sich in einer lokalisierten oder u. U. lebensbedrohlichen generalisierten Soforttypreaktion nach Allergenexposition. In unserem Fall waren sowohl sIgE der CAP-Klasse 1 und eine positive Reaktion im Pricktest gegenüber Weizenmehl nachweisbar. Aufgrund der gastrointestinalen Symptomatik konnte in Zusammenarbeit mit den Gastroenterologen die intestinale Weizenprovokation mittels CLE durchgeführt und die Weizenallergie mittels objektiver Parameter (Leakage, cell shading) gesichert werden. Die CLE ist eine hilfreiche Methode in der Diagnostik der Nahrungsmittelallergien. Die interdisziplinäre Zusammenarbeit zwischen Dermatologen und Gastroenterologen hat für die Diagnostik der Nahrungsmittelallergien einen wichtigen Stellenwert.

\section{P7.3}

\section{Behandlung einer schweren Kuhmilchallergie mit Omalizumab beim Erwachsenen}

\section{B. Klein, J.C. Simon, R. Treudler}

Klinik für Dermatologie, Venerologie und Allergologie, Universitätsklinikum Leipzig, Leipzig, Deutschland

Hintergrund: Die Therapie einer schweren Nahrungsmittelallergie (NMA) besteht bisher im Wesentlichen in einer Allergenkarenz und Notfallbehandlung. Der Einsatz von anti-IgE Antikörpern wird kasuistisch als erfolgsversprechend berichtet.

Kasuistik: Wir berichten über einen 22-jährigen Patienten mit schwerer Kuhmilchallergie mit Z.n. multiplen anaphylaktischen Reaktionen, die seit dem Kleinkindalter bekannt ist und eine Persistenz bis in das Erwachsenenalter mit teils schweren Soforttypreaktionen bei akzidentellem Verzehr zeigte. Der Pricktest auf native Vollmilch war positiv, der CAP-FEIA war ebenfalls positiv für Milcheiweiß (CAP6), Stutenmilch (CAP3), Molke (CAP6), Schafsmilchmolke (CAP6) sowie bos d4, bos d5 und bos d8 (je CAP6) und Schimmelkäse (CAP6), das Gesamt IgE lag bei $1265 \mathrm{kU} / \mathrm{l}$. Nebenbefundlich liegt ein inhalativ gut kontrolliertes Asthma bronchiale vor. Es erfolgte die Einleitung einer off-label Therapie mit Omalizumab ( $3 \times 150 \mathrm{mg} /$ Monat). Unter einer solchen mehrjährigen Therapie führten wir im Verlauf eine doppelblinde orale Expositionstestung auf Kuhmilch bei dem Patienten durch. Dabei konnten $10 \mathrm{ml}$ vertragen werden, nach $30 \mathrm{ml}$ kam es zu Urtikaria, Dyspnoe und Angioödem.

Fazit: Unter einer Therapie mit Omalizumab war eine Erhöhung der Toleranzschwelle für Kuhmilch bei unserem Patienten möglich. Reaktionen bei akzidentellem Verzehr konnten in Folge verhindert werden. Ein weiterer Einsatz von anti-IgE, z. B. auch mit Ligelizumab scheint aussichtsreich.

\section{P7.4}

Nahrungsmittel-Allergien gegen Nüsse, Samen und Hülsenfrüchte im Kindesalter: Relevanz einer Screening-Diagnostik auf seltene Allergene

\section{N. Fischedick, W. G. van Kessel, S. Flümann, C. Koerner-Rettberg}

Klinik für Kinder- und Jugendmedizin, Marien-Hospital Wesel, Wesel, Deutschland

Einleitung: Nüsse und Hülsenfrüchte sind die häufigsten Anaphylaxie-auslösenden Nahrungsmittel im Kindesalter. Erd- und Haselnüsse stehen zahlenmäßig im Vordergrund; seltener werden Allergien gegen Cashew, Pistazie, Paranuss, Pekannuss, Walnuss, Mandel, Macadamia, Sesam und Pinienkerne beobachtet. Es gibt wenig Daten zur Allergiehäufigkeit auf diese Nahrungsmittel bei Kindern mit erhöhtem Allergierisiko. Die diagnostische Austestung ist aufwändig, und praktizierte Testungsstrategien unterscheiden sich zwischen Zentren. Es ist umstritten, ob nach Allergien auf seltene Nüsse/Samen gescreent werden sollte.

Methodik: Retrospektive Analyse der Allergie-Inzidenz auf seltene Nüsse u. Samen an einem Kollektiv atopischer Kinder, die am Marien-Hospital Wesel aufgrund des V. a. Nahrungsmittelallergie oder Sensibilisierungsnachweis bei unklarer Verträglichkeit einer Allergiediagnostik mittels Prick- und CAP-Test 
sowie Klärung der Sensibilisierungsrelevanz durch orale Provokation unterzogen wurden. Ko-Sensibilisierungsmuster und der Effekt des Screenings auf seltene Nüsse/Samen auf das klinische Management werden untersucht.

Ergebnisse: Es wurden bei 475 Pat. 281 Provokationen durchgeführt (122 Erdnuss, 77 Haselnuss, 37 Mandel, 32 Walnuss, 20 Cashew, 6 Macadamia, 5 Pistazie, 4 Pekannuss, 2 Sesam). Bei 10 von 42 Pat. mit Provokations-bestätigter Erdnuss-Allergie fand sich eine zusätzliche Provokations-bestätigte Allergie auf Haselnuss oder Walnuss. Bei 11 von 18 Patienten mit Provokations-bestätigter Haselnuss-Allergie bestand eine zusätzliche Provokations-belegte Allergie auf Erdnuss, Cashew, Pistazie, Macadamianuss oder Walnuss.

Schlussfolgerung: Die Inzidenz von Allergien auf weitere Nüsse oder Samen ist bei Patienten mit Erdnuss- und Haselnuss-Allergie kein seltenes Phänomen. Ein Screening auf Sensibilisierungen mit nachfolgender Provokationstestung identifiziert diese und führt mitunter zu Änderungen in der Beratung bzgl. Diätempfehlungen und Notfallset-Verordnung.

\section{P7.5}

\section{Einfluss der Behandlung mit Palforzia} auf die Symptomschwere verschiedener Systemorganklassen

\section{S. Zeitler', S. Butovas', Lu. Klimek² ${ }^{2}$ C. Vogelberg ${ }^{3}$, A. Kleinheinz ${ }^{4}$, D. Norval ${ }^{5}$, R. Ryan ${ }^{5}$}

${ }^{1}$ Aimmune Therapeutics, München, Deutschland; 'Zentrum für Rhinologie und Allergologie, Wiesbaden, Deutschland:

${ }^{3}$ Klinik und Poliklinik für Kinder- und Jugendmedizin, Universitätsklinik Dresden, Dresden, Deutschland; ${ }^{4}$ Elbe Klinikum Buxtehude, Buxtehude, Deutschland; ${ }^{5}$ Aimmune Therapeutics, London, United Kingdom

Hintergrund: Erdnussallergie ist eine schwere Erkrankung, die ca. $8 \%$ der Kinder mit zunehmender Inzidenz in Industrieländern betrifft. Mit dem vor kurzem von FDA und EMA zugelassenen Produkt Palforzia besteht eine neue und innovative Möglichkeit der Behandlung der Erdnussallergie bei Kindern und Jugendlichen im Alter von 4-17 Jahren. Wie zulassungsklinische Studien (ARC003+ARC004 and ARC010) zeigen, tolerieren die meisten der behandelten Kinder 300, 600 und 1000 mg von AR101 nach Studienabschluss mit moderaten bis gar keinen Symptomen. Jedoch, Toleranz ist ein komplexer Endpunkt und kann daher nicht als Patienten-relevant dargestellt werden. Hinzu, Anteil der symptomfreien Patienten in den klinischen Studien wurde noch nicht untersucht. Ziel dieser Arbeit ist eine zusätzliche Wirksamkeitsanalyse durchzuführen, um Vorkommen und Schwere der Symptome, ohne der allergischen Reaktionen als Krankheitsmanifestation, nach Systemorganklasse auszuwerten.

Methode: Für diese Analyse wurden Daten der Studien im Blick auf die Manifestation von gastrointestinalen, kardiovaskulären/neurologischen und Hautsymptomen untersucht, nach Symptomschwere eingestuft und mit der Placebo-Gruppe verglichen.

Ergebnisse: In der AR101-Gruppe war der Anteil symptomfreier Patienten in allen Systemorgan-Klassen höher im Vergleich zu Placebo (85,8\% gg. 66,1\%; 81,7\% gg. 46\%; 97,3\% gg. $86,3 \%$ und $89,8 \%$ gg. $69,4 \%$ ). Zusätzlich zeigte sich eine signifikante Reduktion des relativen Risikos (RR) der Symptome ( $\geq$ mild) in allen Systemorgan-Klassen in der AR101-Gruppe:
$(0,4206 \quad[0,2964-0,597], \quad p<0,0001 ; 0,3383 \quad[0,2584-0,4429]$, $p<0,0001 ; 0,1739[0,0773-0,391], \quad p<0,001 ; 0,3333[0,2232-$ $0,4978], p<0,0001$ ).

Schlussfolgerung: Eine Behandlung mit AR101 führt zu einem höheren Anteil an symptomfreien Patienten und zu einer signifikanten Reduktion der Symptomschwere in allen Organklassen. Diese Analyse stellt zusätzliche Wirksamkeitsaspekte der Therapie, die höhere klinische Relevanz für Patienten haben könnte, dar.

\section{P7.6}

\section{Sicherheit des Erdnuss(Arachis Hypogaea)- Allergenpulvers PALFORZIA $₫$ bei Kindern und Jugendlichen mit Erdnussallergie}

\section{K. Beyer'1, K. Blümchen², S. Yürek' ${ }^{1}$, A. Fischl², D. Norval ${ }^{3}$, M. Guschmann ${ }^{4}$, S. Zeitler ${ }^{5}$}

${ }^{1}$ Charité Universitätsmedizin Berlin, Berlin, Deutschland; ${ }^{2}$ Universitätsklinikum Frankfurt, Goethe Universität; Frankfurt, Deutschland;

${ }^{3}$ Aimmune Therapeutics, a Nestlé Health Science company, Brisbane, CA, USA;

${ }^{4}$ Institut für klinische Pharmakologie, Berlin, Deutschland; ${ }^{5}$ Aimmune Therapeutics Germany $\mathrm{GmbH}$, München, Deutschland

Analyse gepoolter Daten klinischer Studien über einen Zeitraum von ca. 3,5 Jahren

Das Erdnuss- (Arachis hypogaea) Allergenpulver PALFORZIA $^{\circ}$ ist von FDA und EMA als orale Immunotherapie zur $1 \times$ täglichen Einnahme für Kinder und Jugendliche im Alter von 4-17 Jahren mit Erdnussallergie zugelassen. Nachstehend dargestellt werden die Langzeitsicherheitsdaten von PALFORZIA ${ }^{\oplus}$ nach einer Behandlungszeit von ca. 3,5 Jahren. Gesammelt wurden Daten von 6 klinischen Studien. Insgesamt wurden 1127 erdnussallergische Patienten im Alter zwischen 4-17 Jahren erfasst. Bis Juli 2020 erhielten 755 Studienteilnehmer mindestens 1 Jahr lang PALFORZIA ${ }^{\circ}, 431$ bzw. 94 erhielten das Erdnusspulver über einen Zeitraum von 2 bzw. 3 Jahren. Insgesamt beendeten 13,3\% $(n=150)$ der PALFORZIA ${ }^{\oplus}$-Patienten die Behandlung, meist aufgrund unerwünschter Ereignisse. Diese traten in der Regel innerhalb der ersten 6 Monate auf und betrafen am häufigsten den Gastrointestinaltrakt. Milde bis moderate behandlungsbedingte unerwünschte Ereignisse (TRAEs) zeigten sich ebenfalls primär zu Beginn der Behandlung. Die häufigsten TRAEs waren eine Reizung des Rachenraumes, abdominelle Schmerzen und oraler Juckreiz. Die Rate an systemisch-allergischen Reaktionen jeglicher Schweregrade sowie die Anwendung von Adrenalin war bereits im ersten Behandlungsjahr gering und sank zudem in den Jahren 2 und 3. Über den Zeitraum von 3,5 Jahren zeigten 14 Patienten (1.2\%) eine behandlungsbedingte schwere systemisch-allergische Reaktion. Diese stand meist in Zusammenhang mit Kofaktoren, wie etwa körperlicher Aktivität. Bei den hier präsentierten Daten handelt es sich um die mit 3,5 Jahren umfangreichsten Langzeitsicherheitsdaten bei der Behandlung einer Erdnussallergie. 
Immunomodulatory effects of pectin supplementation depends on the mouse strain and pectin characteristics

\section{F. Blanco-Pérez ${ }^{1}$, H. Steigerwald ${ }^{1}$, M. Krause ${ }^{1}$, M. Albrecht' ${ }^{1}$ D. Stoll ${ }^{2}$, S. Schülke ${ }^{1}$, H.-U. Endreß ${ }^{3}$, S. Kulling ${ }^{2}$, M. Huch ${ }^{2}$, S. Vieths ${ }^{1}$, S. Scheurer ${ }^{1}$}

${ }^{1}$ Paul-Ehrlich-Institut (PEI), Federal Institute for Vaccines and Biomedicines. Langen, Germany;

${ }^{2}$ Max-Rubner-Institut (MRI), Federal Research Institute of

Nutrition and Food, Karlsruhe, Germany;

${ }^{3}$ Herbstreith \& Fox KG, Neuenbürg, Germany

Pectin is a dietary fiber widely used in processed foods to generate gels or modulate viscosity but also acts as prebiotic. It is classified due to its degree of esterification (DE). Pectin from different sources such as apple or citrus serves as carbon source for beneficial gut bacteria and its fermentation might generate short chain fatty acids. A beneficial effect on different diseases such as IgE-mediated food- and respiratory allergy is discussed, either by direct interaction with immune cells or indirectly by modulation of the gut microbiota.

Therefore, we aimed at evaluating the immunomodulatory effect of pectin supplementation in mice. Three mouse strains (CBA, BALB/c and C57BL/6) were fed with different amounts of either apple pectin (AP; DE $57 \%$ ), citrus pectin (CP; DE $7.3 \%$ ) or control diet (CD; $20 \%$ cellulose) for 2 weeks. Feces were collected and mice were monitored daily in regard of their body weight, food consumption and general health status.

Each mouse strain showed different effects associated to the pectin supplementation. CBA had a reduced body weight gain, and enlarged small, large intestine with $15 \% \mathrm{AP}$. BALB/c showed an increased body weight gain with the $\mathrm{CP}$ and a shorter small intestine. C57BL/6 had no effects on the body weight or intestine size.

Pectin led to remarkable changes in the gut microbiota, e.g. AP supplementation shifted microbiota balance towards Bacterioidetes. In addition, AP showed immunomodulatory effects on the serum total IgA, IgG and IgE as well as an enlarged caecum in all mouse strains.

In conclusion, our results suggest that not only pectin characteristics are important to evaluate its effects on the microbiota, but also the mouse strain plays an important role. This needs to be taken into consideration when the effects of pectins are studied in mouse models.

Funding: BMWi, German Federal Ministry for Economic Affairs and Energy, AIF20528.

$B M B F$, German Federal Ministry for Education and Research, O1EA1901
Pektin - ein Risiko für Allergiker? Risikobewertung zu LTP in Pektin-Varianten

\section{H. Steigerwald ${ }^{1}$, F. Blanco-Perez ${ }^{1}$, M. Albrecht ${ }^{1}$, C. Bender ${ }^{2}$, M. Bunzel ${ }^{2}$, H.-U. Endreß ${ }^{3}$, S. Scheurer ${ }^{1}$, S. Vieths ${ }^{1}$}

${ }^{1}$ Paul-Ehrlich-Institut, Bundesinstitut für Impfstoffe und biomedizinische Arzneimittel, Langen, Deutschland; ${ }^{2}$ Karlsruher Institut für Technologie, Karlsruhe, Deutschland; ${ }^{3}$ Herbstreith \& Fox KG Pektin-Fabriken, Neuenbürg, Deutschland

Pektin, ein Polysaccharid aus den Zellwänden höherer Pflanzen, findet Anwendung in Bereichen wie Pharma-, Kosmetikund Lebensmittelindustrie und wird vor allem aus Früchten, meist Äpfeln und Zitrusfrüchten gewonnen. In der Nahrungsmittelindustrie findet es vor allem aufgrund seiner technologischen Eigenschaften Einsatz als Gelier- und Verdickungsmittel. Durch seine präbiotischen und immunmodulatorischen Effekte gewinnt der Ballaststoff jedoch immer mehr an Bedeutung. Auch der Einfluss auf die Entwicklung von allergischen Erkrankungen steht im Fokus mehrerer Studien. Daher muss besonders bei Nahrungsmittelallergikern die Gefahr einer allergischen Reaktion durch Rückstände von Allergenen im Pektin ausgeschlossen werden. $\mathrm{Zu}$ diesen zählen bspw. Lipid-Transfer-Proteine (LTPs) - die hitzestabilen Hauptallergene in vielen Früchten, darunter Äpfel und Zitrusfrüchte.

Um das Risiko für Patienten, die auf LTPs aus Früchten reagieren, abschätzen zu können, haben wir zwei Pektine aus Apfeltrester und Zitrusschalen in Bezug auf LTP Rückstände charakterisiert.

Es konnte gezeigt werden, dass die optimale Methode zur Detektion von Proteinrückständen stark von der chemischen Struktur der jeweiligen Pektine abhängt. Zudem ist ein vorheriger enzymatischer Verdau notwendig, um Proteine in der Pektin-Matrix nachweisen zu können. Spiking-Experimente mit LTPs lassen darauf schließen, dass die Menge des möglicherweise im Apfelpektin enthaltenen Allergens nicht ausreicht, um anaphylaktische Reaktionen hervorzurufen. Durch Fällung des Pektins mit Kalziumchlorid konnte dies auch für das verwendete Zitruspektin gezeigt werden.

Diese Daten deuten darauf hin, dass die untersuchten Pektine auch beim Verzehr höherer Mengen kein relevantes Risiko für eine anaphylaktische Reaktion durch LTPs darstellen.

Finanzierung: BMWi, Bundesinstitut für Wirtschaft und Energie, AIF20528

$B M B F$, Bundesministerium für Bildung und Forschung, $01 E A 1901$

\section{P7.9}

Erstmanifestation einer Tropomyosin-vermittelten Soforttypallergie durch Verzehr essbarer Insekten

\section{S. Hofmann', N. Gäbelein-Wissing ${ }^{2}$}

${ }^{1}$ Helios Universitätsklinikum Wuppertal, Zentrum für Dermatologie, Allergologie und Dermatochirurgie, Universität Witten/Herdecke, Deutschland;

${ }^{2}$ Dermatologische Praxis, Wuppertal, Deutschland 
Ein einem 27-jährigen Patienten sei es erstmals nach Verzehr von gerösteten Hausgrillen (Acheta domesticus) zu Parästhesien an der Oberlippe gekommen. Wenige Tage später verzehrte der Patient Garnelen in einem Reisgericht mit Tomatensoße und Gemüse. Innerhalb weniger Minuten habe er erneut Parästhesien an der Lippe bemerkt und im Verlauf eine generalisierte Urtikaria entwickelt. Unter Verzicht auf Garnelen traten keine erneuten allergischen Reaktionen auf.

Die serologische Diagnostik ergab ein Gesamt-IgE von 176 $\mathrm{kU} / \mathrm{l}$ bei negativem fx5-Nahrungsmittelscreening. Eine Sensibilisierung gegenüber dem Hausstaubmilben-Tropomyosin Der $p$ 10 war mit $0,4 \mathrm{kU} / \mathrm{l}(\mathrm{Norm}<0,35)$ nachweisbar ebenso wie ein erhöhtes IgE gegenüber Shrimps $(1,78 \mathrm{kU} / \mathrm{l})$. Das spezifische IgE für das Majorallergen der Hausstaubmilbe (Der p 1) war normwertig.

In der Pricktestung zeigten sich positive Reaktionen für Dermatophagoides pteronyssinus $(3 / 5 \mathrm{~mm})$, Dermatophagoides farinae $(3 / 4 \mathrm{~mm})$ und Krabbe $(3 / 5 \mathrm{~mm}$; Extrakte der Firma ALK-Abello, Hamburg). Der Prick-to-Prick-Test mit Shrimps ergab eine deutlich positive Reaktion $(5 / 30 \mathrm{~mm})$, während die anderen Komponenten des Reisgerichts keine Reaktion hervorriefen.

In Anbetracht des stetigen Weltbevölkerungswachstums stellen essbare Insekten für Mensch und Nutztiere ein interessantes, proteinreiches Nahrungsmittel dar. Jedoch besteht ein allergenes Potenzial aufgrund einer möglichen IgE-Kreuzreaktivität zwischen Schalentieren und Grillen, Wüstenheuschrecken sowie gelben Mehlwürmern. Neben den Tropomyosinen sind auch $\alpha$-Amylasen und ein bisher nicht identifiziertes 23 kDa-großes Protein allergologisch relevant, insbesondere bei Patienten mit Hausstaubmilbenallergie.

Unseres Wissens ist dies der erste Fall einer allergischen Reaktion vom Soforttyp nach Verzehr von Hausgrillen. In Anbetracht des aktuellen Trends zum Verzehr essbarer Insekten sollten Hausstaubmilben- und Schalentierallergiker bezüglich möglicher Kreuzreaktionen gewarnt werden.

\section{P7.10}

Kabeljau als Auslöser einer FDEIA - ein Fallbericht

V. Faihs', C. Hilger', A. Kuehn², S. Mathes' ${ }^{1}$, L. Li', T. Nau', C. Kugler', K. Brockow', T. Biedermann', U. Darsow ${ }^{1}$

${ }^{1}$ Klinik und Poliklinik für Dermatologie und Allergologie am Biederstein, Technische Universität München, München, Deutschland;

${ }^{2}$ Luxembourg Institute of Health, Department of Infection and Immunity, Esch-sur-Alzette, Luxemburg

Hintergrund: Eine nahrungsmittelabhängige, anstrengungsinduzierte Anaphylaxie (food-dependent exercise-induced anaphylaxis; FDEIA) ist eine IgE-vermittelte Anaphylaxie, die erst in Kombination mit Kofaktoren auftritt, z.B. körperlicher Anstrengung. Eine Fehldiagnose als idiopathische Anaphylaxie ist häufig und führt oft zusätzlich zu einer schweren psychischen Belastung für die Patienten.

Kasuistik: Ein 12-jähriger Patient mit bekannter Rhinokonjunktivitis allergica und Asthma bronchiale entwickelte beim Fußballspielen direkt nach dem Verzehr eines fischhaltigen Gerichts eine generalisierte Urtikaria. Beim Verzehr von Meeresfrüchten sowie Thunfisch bestünde enoraler Juckreiz, alle anderen Lebensmittel würden vertragen. In der allergologischen Abklärung zeigten sich Sensibilisierungen gegen Kabeljau (Prick $3 \mathrm{~mm} / 4 \mathrm{~mm}$, spez. IgE 0,35 kU/l) sowie Thun- fisch (spez. IgE 2,92 kU/l). Es war kein spez. IgE gegen die Parvalbumine, Enolase und Aldolase des Kabeljaus nachweisbar (Gad cl, Gad ml-3<0,1 kU/l). Zusätzlich fanden sich Sensibilisierungen gegen Krusten- und Schalentiere (Prick, spez. IgE) inkl. Tropomyosin der Garnele (rPen al > $100 \mathrm{kU} / \mathrm{l}$ ). In der oralen Provokationstestung wurde Kabeljau bis kumulativ $124 \mathrm{~g}$ zunächst problemlos vertragen. Am nächsten Tag wurde in Kombination mit Sport provoziert. Nach Verzehr von $70 \mathrm{~g}$ Kabeljau (kum. $95 \mathrm{~g}$ ) und anschließender, 15-minütiger anaerober Belastung auf dem Laufband entwickelten sich multiple, stecknadelkopfgroße Urticae, die mit Dimetinden und Prednisolon therapiert wurden.

Kommentar: Bei unserem Patienten wurde die Diagnose einer anstrengungsinduzierten Kabeljauallergie gesichert, welche nicht durch Gad c1 oder Gad m1-3 ausgelöst wurde. Obwohl Fisch ein häufiges Allergen darstellt, ist es ein seltener Auslöser einer FDEIA; bisher sind nur wenige Fallberichte publiziert. Durch die Diagnosestellung und Meidung von Fisch im Zusammenhang mit Kofaktoren kann unser sportlicher Patient in Zukunft potenziell gefährliche Anaphylaxien verhindern.

\section{Other}

\section{P4.2}

Pharmakokinetik in der Therapie der allergischen bronchopulmonalen Aspergillose (ABPA)

\section{A. Scholz, S. Huber, S. Schmidt}

Kinder- und Jugendmedizin der Universität Greifswald, Greifswald, Deutschland

Fall: 16-jähriger Patient mit Zystischer Fibrose mit Lungenund Darmbeteiligung, Z.n. Leber-Tx (Immunsuppression mit Tacrolimus) und Nierenfunktionseinschränkung stellt sich zur antibiotischen Eradikation bei pulmonalem Erstnachweis von Pseudomonas aeruginosa und deutlicher Verschlechterung der Lungenfunktion vor.

Die antibiotische Therapie brachte keine Besserung. Im Röntgen-Thorax sah man ein flaues, rundlich begrenztes Infiltrat im Mittellappen rechts. Im CT Thorax zeigten sich sacculäre Bronchiektasien im rechten Oberlappen, mediastinale Lymphknotenschwellung und es erhärtete sich der V.a. eine Aspergillose bei Gesamt IgE von 4580 und rekombinantem Allergen rAsp f4 von 18,1 und rAsp f6 0,23, Aspergilus IgE. 51,6.

Die weiteren Diagnosekriterien für eine ABPA als Hypersensitivitätserkrankung der Lunge waren erfüllt.

Therapie: Aufgrund des Nachweises von Aspergillus fumigatus im Bronchialsekret wurde eine Therapie mit Itraconazol begonnen. Itraconazol wird hauptsächlich über das Cytochrom CYP3A4 metabolisiert. Es werden sequenziell 3 ItraconazolMetabolite gebildet. Diese tragen signifikant zur starken CYP3A4-Hemmung bei. Somit führt die gleichzeitige Gabe von Itraconazol mit CYP3A4-Substraten wie Tacrolimus zu einer Substraterhöhung im Plasma. Daher wurde mit Beginn der Itraconazoltherapie die Dosierung von Tacrolimus reduziert und anfangs engmaschig überwacht. Zusätzlich wurde mehrmals eine Methylprednisolon-Stoßtherapie durchgeführt, um die Entstehung eines Diabetes mellitus bei bereits bestehender gestörter Glukosetoleranz zu verhindern. Unter dieser Therapie besserten sich Lungenfunktion, serologische Parameter und radiologische Zeichen. Die Itraconazol- und Tacrolimus-Spiegel lagen stets im beabsichtigten Bereich. 
Zusammenfassung: Eine ABPA erfordert in besonderen Situationen ein multidisziplinäres Management.

\section{P4.5}

\section{Kein Einfluss atopischer Erkrankungen auf den Verlauf oder die Schwere einer COVID-19- Erkrankung}

\section{J. Zarnowski, P. Kage, J.-C. Simon, R. Treudler}

Klinik für Dermatologie, Venerologie und Allergologie, Universitätsklinikum Leipzig, Leipzig, Deutschland

Hintergrund: In der Literatur sind Assoziationen zwischen Th-2-dominierter Immunantwort und Verminderung der viralen Immunabwehr beschrieben worden. Ebenso konnten erste Studien eine geschwächte virale Abwehr sowie erhöhte SARS-CoV-2-Infektionsraten während eines erhöhten Pollenfluges zeigen. Ziel unserer Arbeit war es den Einfluss atopischer Vorerkrankungen auf die Schwere und den Verlauf einer COVID-19-Erkrankung in einer Kohorte Leipziger Patienten zu untersuchen.

Methoden: In dieser retrospektiven Studie erfassten wir demografische Daten sowie selbstberichtete Angaben in Hinblick auf die COVID-19-Erkrankung (Symptome, Regenerationszeit) sowie atopische Vorerkrankungen mittels intern konzipierten Fragebogen. Die Patienten wurden nach bestätigter und überstandener SARS-CoV-2 Infektion entweder über ein Anschreiben des Gesundheitsamtes oder aus der allergologischen Sprechstunde rekrutiert.

Ergebnisse: Von 107 Patienten gaben 53 (49.5\%, mittleres Alter: $44.4 \mathrm{~J})$ eine atopische Erkrankung an während 54 (50.5\%, mittleres Alter: $44.5 \mathrm{~J}$ ) als gesunde Kontrollprobanden dienten. In unserer Erhebung zeigten sich keine signifikanten Unterschiede in der Alters- und Geschlechtsverteilung beider Patientengruppen. 104/107 (97.2\%) aller Patienten waren symptomatisch während der COVID-19-Erkrankung. Zwischen beiden Patientengruppen zeigte sich kein signifikanter Unterschied zwischen der Häufigkeit aller berichteten Symptome sowie in der berichteten Regenerationszeit, welche in $69 \%$ (Kontrollprobanden) bzw. $74 \%$ (Atopiker) <2 Wochen betrug. Ebenso zeigte sich eine vergleichbare Hospitalisierungsrate zwischen beiden Patientengruppen mit jeweils $5.7 \%$ vs. $5.6 \%$.

Schlussfolgerung: In unserer Erhebung zeigte sich kein Einfluss atopischer Erkrankungen auf die Schwere, Hospitalisierungsrate oder Regenerationszeit nach überstandener COVID-19-Erkrankung. Dies ist vergleichbar mit international publizierten Daten, die Th2-dominierte Erkrankungen meist nicht als Risikofaktor identifizieren konnten.

\section{P4.9}

\section{Allergie auf Glukosesensoren - Problem gelöst?}

\section{T. G. Nau, C. Schönmann, S. Mathes, Va. Faihs, S. Schneider, T. Biedermann, K. Brockow, U. Darsow}

Klinik und Poliklinik für Dermatologie am Biederstein, München, Deutschland

Hintergrund: Auf der Haut angebrachte Sensoren, die ein kontinuierliches Blutglukose-Monitoring erlauben, werden immer beliebter. Da diese nun verstärkt verwendeten Geräte über längere Zeit auf der Haut angebracht sind, mehren sich auch die Fälle von Hautreaktionen gegenüber einzelnen Komponenten dieser Systeme. Bei dem meist verwendeten Sensor-Modell FreeStyle Libre (FSL) wurde 2017 Isobornylacrylat (IBOA) als das verantwortliche Hauptallergen identifiziert. Das Dexcom (DC)-Glukose-Monitoring-System (Nintamed) stellt eine IBOA-freie Alternative für Diabetes-Patienten dar.

Kasuistik I: Eine 24-jährige Typ 1-Diabetikerin berichtet von einer erythematösen Plaque mit starkem Juckreiz unter dem FSL, welche 4 Wochen nach Erstapplikation aufgetreten sei, sodass die Pat. den Sensor in Woche 5 habe abnehmen müssen. Daraufhin Umstellung auf DC, wobei die Pat. in Woche 5 erneut eine nässende, erythemat. Plaque unter dem Sensor entwickelte. Im Epikutantest (Standardreihe, Kunstharze/Kleber, Kunststoffe, DC Klebefläche) zeigt sich nur die Klebefläche des DC positiv, IBOA blieb negativ.

Kasuistik II: Eine 64-jährige Typ 2-Diabetikerin stellt sich mit starkem Juckreiz und Erythemen an den Auflageflächen ihres DC-Sensors vor. Diese Symptome träten jeweils 4 Tage nach Aufbringen des Systems auf. Wir führten eine Epikutantestung mit den Reihen Standard, Kunstharze/Kleber, Kunststoffe, Barrierepflastern, dem grauen Kunststoffanteil des DC und dem DC-Kunststoff-Bügel durch. In der 48-h-Ablesung zeigten sich keine positiven Reaktionen, in der 72- und 96-h-Ablesung imponierte lediglich der DC-Kunststoff-Bügel deutlich positiv, nicht jedoch IBOA.

Kommentar: Auch IBOA-freie Systeme können allergische Kontaktekzeme hervorrufen. Das genaue, im DC-KunststoffBügel enthaltene Allergen bleibt unbekannt. Eine Identifikation der relevanten Allergene und die allergologische Diagnostik bei Kontaktallergie auf Glukose-Sensoren sind essenziell, um frühzeitig auf ein verträgliches Modell umzustellen.

\section{P5.10}

Vergleichende Untersuchung der Wirksamkeit und Verträglichkeit von Ectoin $₫$ Mouth and Throat Spray Althaea Honey (ERS09) und einem Salzspray bei Patienten mit akuter Pharyngitis und trockenem Husten

\section{Roventa' ${ }^{1}$, U. Pieper-Fürst ${ }^{2}$, C. Acikel $^{2}$, D. Santos ${ }^{3}$, U. Sent ${ }^{3}$, R. Mösges ${ }^{1,2}$}

${ }^{1}$ Institut für Medizinische Statistik und Bioinformatik (IMSB), Medizinische Fakultät, Universität Köln, Deutschland; ${ }^{2}$ ClinCompetence Cologne $\mathrm{GmbH}$, Köln, Deutschland ${ }^{3}$ Sanofi-Aventis Deutschland GmbH, Frankfurt am Main, Deutschland

Einleitung: Die Wirksamkeit und Verträglichkeit des ERS09-Sprays mit seinen zellschützenden und entzündungshemmenden Bestandteilen wurde im Vergleich mit einem etablierten Salzspray bei akuter Pharyngitis und trockenem Husten untersucht.

Methoden: NCT04203810 ist eine multizentrische, aktivkontrollierte, randomisierte, offene $\S 23 \mathrm{~b}$ MPG-Studie durchgeführt in Deutschland mit 2 parallelen Gruppen und 193 Patienten mit Halsschmerzen aufgrund akuter Pharyngitis und trockenem Husten. Patienten erhielten entweder ERS09 oder ein Salzspray und wurden über einen Zeitraum von $7 \pm 2$ Tagen untersucht.

Ergebnisse: Der im Tagebuch erfasste Total Symptom Score (TSS) mit den Unterscores Halsschmerzintensität, Schluckbeschwerden und Intensität des trockenen Hustens wurde durch 
beide Behandlungen effektiv reduziert. Die Mehrheit von Patienten mit einer 50 \%igen Reduktion des TSS kamen jedoch in der ERS09-Behandlungsgruppe vor und zwar an 6 Tagen der Studiendauer von $7 \pm 2$ Tagen. Der Anteil von Patienten mit einer Verbesserung der Schwellung bzw. Rötung des Pharynx war um 5,9\% bzw. 5,8 \% höher in der ERS09-Gruppe als in der Vergleichsgruppe. Die allgemeine Wirksamkeit des Sprays wurde durch einen höheren Anteil der Ärzte (6,2 \%) und Patienten $(5,9 \%)$ in der ERS09-Gruppe als gut oder sehr gut als in der Vergleichsgruppe bewertet. Der Anteil von Patienten, die innerhalb der ersten 5 Minuten nach initialer Anwendung des Sprays Linderung verspürten, war um 11,1\% höher in der ERS09Gruppe. Die Verträglichkeit wurde in beiden Gruppen überwiegend als gut oder sehr gut bewertet. Im Vergleich zur SalzsprayGruppe würde ein 11,7\% höherer Anteil von Patienten in der ERS09-Gruppe das Spray in der nächsten Erkältungssaison wieder benutzen und ein 8,5\% höherer Anteil von ERS09-Patienten würde das Spray Familie und Freunden empfehlen.

Fazit: Beide Sprays stellen eine wirksame und gut verträgliche, nicht-pharmakologische Therapieoption dar, wobei die Wirksamkeit in der ERS09-Gruppe etwas besser erschien.

\section{P7.11}

\section{Unverträglichkeitsreaktionen im Kontext der COVID-19-Impfung - Erfahrungen aus den zertifizierten Allergiezentren in Deutschland in Kooperation mit Allergieabteilungen und Schwerpunktpraxen}

\section{Worm 1 , A. Bauer', J. Fluhr³, W. Pfützner', B. Wedi ${ }^{5}$, L. Klimek ${ }^{6}$, A. Jung ${ }^{7}$, T. Jakob ${ }^{7}$, R. Treudler, G. Wurpts ${ }^{9}$, H. Dickel ${ }^{10}$, B. Espey ${ }^{10}$, U. Raap ${ }^{11}$, R. Brehler ${ }^{12}$, N. Mülleneisen ${ }^{13}$, T. Buhl ${ }^{14}$, K. Brockow ${ }^{15}$}

${ }^{1}$ Allergologie und Immunologie, Klinik für Dermatologie, Venerologie und Allergologie, Charité - Universitätsmedizin Berlin, corporate member of Freie Universität Berlin and Humboldt-Universität zu Berlin, Berlin, Deutschland; ${ }^{2}$ Klinik und Poliklinik für Dermatologie, Universitätsklinikum Carl Gustav Carus Dresden, Technische Universität Dresden, Deutschland;

${ }^{3}$ Dermatologische Allergie-Folgenforschung, Campus Benjamin-Franklin, Charité - Universitätsmedizin Berlin, corporate member of Freie Universität Berlin and HumboldtUniversität zu Berlin, Berlin, Deutschland;

${ }^{4}$ Klinik für Dermatologie und Allergologie, $\mathrm{AZH}$,

Universitätsklinikum Marburg, Marburg, Deutschland; ${ }^{5}$ Klinik für Dermatologie, Allergologie und Venerologie Comprehensive Allergy Center (CAC) Behandlungszentrum für Hereditäre Angioödeme im ZSE, Medizinische Hochschule Hannover, Hannover, Deutschland; ${ }^{6}$ Zentrum für Rhinologie und Allergologie, Allergiezentrum Wiesbaden/Rhein-Main, Wiesbaden, Deutschland; ${ }^{7}$ Klinik für Dermatologie und Allergologie Universitätsklinikum Gießen, Gießen, Deutschland;

${ }^{8}$ Klinik für Dermatologie, Venerologie und Allergologie, Universitätsmedizin Leipzig, Leipzig, Deutschland; ${ }^{9}$ Klinik für Dermatologie und Allergologie, Aachener Comprehensive Allergy Center, Aachen, Deutschland;
${ }^{10} \mathrm{Klinik}$ für Dermatologie, Venerologie und Allergologie, St. Josef-Hospital, Universitätsklinikum der Ruhr-Universität Bochum, Bochum, Deutschland;

${ }^{11}$ Allergiezentrum Oldenburg, Klinikum Oldenburg AöR, Universitätsklinik für Dermatologie und Allergologie,

Oldenburg, Deutschland;

${ }^{12} \mathrm{Ambulanz}$ für Allergologie, Berufsdermatologie und Umweltmedizin, Universitätsklinikum Münster, Münster, Deutschland;

${ }^{13}$ Asthma und Allergiezentrum Leverkusen, Leverkusen, Deutschland;

${ }^{14}$ Allergiezentrum Südniedersachsen, Universitätsmedizin Göttingen, Göttingen, Deutschland;

${ }^{15}$ Hautklinik Campus Biederstein, Klinikum rechts der Isar, Technische Universität München, München, Deutschland

Einleitung: Seit Dezember 2020 wird in Deutschland zur Überwindung der COVID-19 Pandemie die Bevölkerung geimpft. Aktuell stehen mRNA-Impfstoffe (BioNTech und Moderna) sowie Adeno-Virus-basierte Impfstoffe (AstraZeneca und Johnson\&Johnson) zur Verfügung. Nachdem in der Literatur über allergische Zwischenfälle im Kontext der COVID19-Impfung berichtet wurde, war es Ziel der Untersuchung ein standardisiertes Vorgehen zur allergologischen Abklärung zu entwickeln und umzusetzen.

Methode: Die Anamnese wurde bezüglich Art und Zeitverlauf der Symptome im Kontext der Impfung erfasst. Darüber hinaus wurde ein standardisiertes allergologisches Vorgehen (Gesamt-IgE, Tryptase, definierte Hauttestungen einschließlich Impfstoffen und deren Inhaltsstoffen) entwickelt und anschließend prospektiv von Februar 2021 bis Juni 2021 erhoben.

Ergebnisse: Es wurden die Daten von 163 Patienten erfasst, davon waren 140 weiblich und 23 männlich. Das durchschnittliche Alter betrug 57,5 Jahre. Die berichteten Symptome reichten von Hautsymptomen, wie Urtikaria und Angioödemen bis zu Dyspnoe und Kreislaufreaktionen, die in den meisten Fällen innerhalb von 30 Minuten nach der Impfung auftraten. Die Haut-Prick-Testungen mit Impfstoffen waren bei über $95 \%$ der Patienten negativ. Hauttests mit Inhaltsstoffen der Impfstoffe wie Polyethylenglykol 2000 und Polysorbat 80 zeigten einzelne positive Lokalreaktionen. Der von einigen Zentren durchgeführte Basophilen-Aktivierungstest war in $>90 \%$ der Untersuchungen negativ. Patienten, die nach allergologischer Abklärung die 2. Impfung (gleicher Impfstoff oder anderer Impfstoff) erhalten haben, haben diese in der Regel vertragen.

Schlussfolgerung: Im Kontext der COVID-19-Impfung kommt es sehr selten zu Sofortreaktionen, die sich an der Haut/ Schleimhaut und noch seltener mit respiratorischen und/ oder kardiovaskulären Symptomen manifestieren. In der Regel haben sich die Symptome bei Betroffenen rasch zurückgebildet und die allergologische Abklärung ergab in der überwiegenden Anzahl der Fälle keinen Hinweis auf ein Typ-I allergisches Geschehen. Aufgrund einzelner positiver Befunde mit PEG2000 bzw Polysorbat 80 scheinen diese Inhaltsstoffe als mögliche Ursache in Frage zu kommen. Die zugrundeliegenden Ursachen von Sofortreaktionen bei Patienten im Kontext der COVID-19-Impfung müssen weiter untersucht werden, um Patienten optimal zu beraten und möglichst vielen eine Impfung zu ermöglichen. 